\title{
A REVIEW OF HEAT TRANSFER PHYSICS
}

\section{V.P. Carey ${ }^{1}$, G. Chen ${ }^{2}$, C. Grigoropoulos ${ }^{3}$, M. Kaviany ${ }^{4}$, and A. Majumdar ${ }^{3}$}

${ }^{1}$ Mechanical Engineering Department, University of Californian, Berkeley, California, USA

${ }^{2}$ Mechanical Engineering Department, Massachusetts Institute of Technology, Cambridge, Massachusetts, USA

${ }^{3}$ Mechanical Engineering Department, University of Californian, Berkeley, California, USA Materials Sciences Division, Lawrence Berkeley National Laboratory, Berkeley, California, USA

${ }^{4}$ Department of Mechanical Engineering, University of Michigan, Ann Arbor, Michigan, USA

With rising science contents of the engineering research and education, we give examples of the quest for fundamental understanding of heat transfer at the atomic level. These include transport as well as interactions (energy conversion) involving phonon, electron, fluid particle, and photon (or electromagnetic wave). Examples are

1. development of MD and DSMC fluid simulations as tools in nanoscale and microscale thermophysical engineering.

2. nanoscale thermal radiation, where the characteristic structural size becomes comparable to or smaller than the radiation (electromagnetic) wavelength.

3. laser-based nanoprocessing, where the surface topography, texture, etc., are modified with nanometer lateral feature definition using pulsed laser beams and confining optical energy by coupling to near-field scanning optical microscopes.

4. photon-electron-phonon couplings in laser cooling of solids, where the thermal vibrational energy (phonon) is removed by the anti-Stokes fluorescence; i.e., the photons emitted by an optical material have a mean energy higher than that of the absorbed photons.

V.P.C. gratefully acknowledges the support for research in this area under NSF Grant No. CTS0456982. G.C. acknowledges contributions from A. Narayanswamy, L. Hu, and Z. Chen and financial support of DOE (DE-FG02-99ER45747) and ONR (N00014-03-1-0835) and ONR MURI on Electromagnetic Metamaterials. C.P.G.'s review is based on work by David J. Hwang and Anant Chimmalgi at the Laser Thermal Laboratory. Financial support by the National Science Foundation under the SINAM NSEC, by DOE, Army and Applied Spectra is gratefully acknowledged. The review by M.K. is based on the Ph.D. research of Mr. Xiulin Ruan and his assistance (including in preparation of this review) is gratefully appreciated as well as the financial support by NSF, DOE, and UM Rackham School of Graduate Studies. A.M. thanks Woochul Kim (School of Mechanical Engineering, Yonsei University, Seoul, Korea) and Robert Wang for their contributions to this review and support from the Basic Energy Sciences, Department of Energy and the Chemical and Transport Systems Division of the National Science Foundation and Office of Naval Research Multidisciplinary University Research Initiative grant with an agency award number: N00014-03-1-0790.

Address correspondence to M. Kaviany, Department of Mechanical Engineering, University of Michigan, Ann Arbor, MI 48109, USA. E-mail: kaviany@umich.edu 
5. exploring the limits of thermal transport in nanostructured materials using spectrally dependent phonon scattering and vibrational spectra mismatching, to impede a particular phonon bands.

These examples suggest that the atomic-level heat transfer builds on and expands electromagnetism (EM), atomic-molecular-optical physics, and condensed-matter physics. The theoretical treatments include ab initio calculations, molecular dynamics simulations, Boltzmann transport theory, and near-field EM thermal emission prediction. Experimental methods include near-field microscopy.

Heat transfer physics describes the kinetics of storage, transport, and transformation of microscale energy carriers (phonon, electron, fluid particle, and photon). Sensible heat is stored in the thermal motion of atoms in various phases of matter. The atomic energy states and their populations are described by the classical and the quantum statistical mechanics (partition function and combinatoric energy distribution probabilities). Transport of thermal energy by the microscale carriers is based on their particle, quasi-particle, and wave descriptions; their diffusion, flow, and propagation; and their scattering and transformation encountered as they travel. The mechanisms of energy transitions among these energy carriers, and their rates (kinetics), are governed by the match of their energies, their interaction probabilities, and the various hindering-mechanism rate (kinetics) limits. Conservation of energy describes the interplay among energy storage, transport, and conversion, from the atomic to the continuum scales.

With advances in micro- and nanotechnology, heat transfer engineering of micro-and nanostructured systems has offered new opportunities for research and education. New journals, including Nanoscale and Microscale Thermophysical Engineering, have allowed communication of new specific/general as well directly useful/educational ideas on heat transfer physics. In an effort to give a more collective perspective of such contribution, here we put together a collection on small-scale heat transfer involving phonon, electron, fluid particle, and photon. These are

1. Development of MD and DSMC fluid simulations as tools in nanoscale and microscale thermophysical engineering (Carey)

2. Nanoscale thermal radiation (Chen)

3. Laser-based nanoprocessing (Grigoropoulos)

4. Photon-electron-phonon couplings in laser cooling of solids (Kaviany)

5. Exploring the limits of thermal transport in nanostructured materials (Majumdar).

KEY WORDS: phonon, electron, fluid particle, photon, heat transfer physics, nano-and micro heat transfer, laser-base nano-processing, laser cooling of solids, nanoscale thermal radiation, molecular dynamics, thermophysical engineering, thermal transport

\section{NOMENCLATURE}

interatomic distance

photon-electron coupling

factor

ion-phonon coupling factor

speed of sound

vapor specific heat at constant

pressure

droplet diameter, $2 r_{d}$

mass diffusion coefficient in

vapor

phonon density of states

macroscopic electric field

static crystal field

polarization vector

force vector

\section{G}

go

$\mathrm{H}$

$h$

$\hbar$

$h_{l v}$

$j, J$

$J_{a}$

$J_{z}$

$J_{Q, i}$ thermal boundary

conductance

force constant between nearest-neighbor atoms quantum of thermal conductance

Hamiltonian

Planck's constant

$h / 2 \pi$

latent heat of vaporization molecular number flux

Jakob number, $c_{p, v}\left(T_{\infty}-T_{d}\right) / h_{l v}$ energy flux in the $z$ direction energy flux at interface 


\begin{tabular}{|c|c|}
\hline$K n_{D}$ & Knudsen number, $\lambda / 2 r d$ \\
\hline $\mathbf{k}$ & reciprocal lattice vector \\
\hline$k_{v}$ & gas thermal conductivity \\
\hline$k_{B}$ & $\begin{array}{l}\text { Boltzmann constant, } \\
1.38054 \times 10^{-23} \mathrm{~J} / \mathrm{K}\end{array}$ \\
\hline$k$ & thermal conductivity \\
\hline$L_{x}, L_{y}, L_{z}$ & $\begin{array}{l}\text { simulation domain dimension } \\
\text { in the } x, y \text {, and } z \text { direction }\end{array}$ \\
\hline $\bar{M}$ & molecular or atomic mass \\
\hline$m$ & mass of a molecule \\
\hline$N$ & $\begin{array}{l}\text { number of molecules in } \\
\text { simulation }\end{array}$ \\
\hline$N_{A}$ & $\begin{array}{l}\text { Avogadro's number, } \\
6.02252 \times 10^{26} \mathrm{kmol}^{-1}\end{array}$ \\
\hline$N_{\text {atom }}$ & number of atoms per molecule \\
\hline$N_{i, j}$ & $\begin{array}{l}\text { number density of phonons in } \\
\text { side } i \text { of polarization }\end{array}$ \\
\hline$N_{m p p}$ & $\begin{array}{l}\text { number of molecules per } \\
\text { particle }\end{array}$ \\
\hline$n$ & number density of atoms \\
\hline$n$ & index of refraction \\
\hline$n_{b}, n_{a}$ & $\begin{array}{l}\text { populations at the excited and } \\
\text { ground states }\end{array}$ \\
\hline$n_{m}$ & number density of molecules \\
\hline$P$ & pressure \\
\hline $\mathbf{P}$ & dipole displacement \\
\hline$P_{c}$ & critical pressure \\
\hline$P_{v e}$ & $\begin{array}{l}\text { equilibrium vapor pressure at } \\
\text { the droplet interface }\end{array}$ \\
\hline$P_{\infty}$ & $\begin{array}{l}\text { pressure in the far ambient } \\
\text { vapor }\end{array}$ \\
\hline$Q_{q}$ & $\begin{array}{l}\text { normal coordinate } \\
\text { displacement }\end{array}$ \\
\hline$q$ & Ewald shielding charge width \\
\hline$q$ & point charge \\
\hline$q$ & wave vector \\
\hline$q$ & heat flux \\
\hline$R$ & gas constant \\
\hline$\Re$ & $\begin{array}{l}\text { a random number uniformly } \\
\text { distributed on }[0,1]\end{array}$ \\
\hline$r, r_{i j}$ & $\begin{array}{l}\text { intermolecular separation } \\
\text { distance }\end{array}$ \\
\hline$r_{c}$ & critical droplet radius \\
\hline$r_{d}$ & radius of droplet \\
\hline$r_{i}$ & interface radius of curvature \\
\hline$r_{s}$ & $\begin{array}{l}\text { radius of droplet, bubble or } \\
\text { sphere }\end{array}$ \\
\hline$r_{w}$ & $\begin{array}{l}\text { wall potential length } \\
\text { parameter }\end{array}$ \\
\hline$r_{0}$ & $\begin{array}{l}\text { Lennard-Jones potential } \\
\text { length parameter }\end{array}$ \\
\hline$S$ & thermopower \\
\hline$\dot{S}_{p h-e-p}$ & net cooling power \\
\hline$T$ & absolute temperature \\
\hline$T_{c}$ & critical temperature \\
\hline$T_{d}$ & droplet temperature \\
\hline
\end{tabular}

$T_{r} \quad$ reduced temperature, $T / T_{c}$

$T_{\infty} \quad$ temperature in the far ambient

$\boldsymbol{u}_{i} \quad$ lattice displacement vector

$\hat{u}_{l v} \quad$ latent energy per water

molecule merged into droplet

$\mathrm{V}_{\text {ion }}(\mathbf{r}) \quad$ potential of the ion on its

valence electron

V volume

$v \quad$ molecular translational

velocity

$X_{w} \quad$ mole fraction of water vapor in gas mixture

$x_{i j}, y_{i j}, \quad$ distance between molecules $i$

$z_{i j}, r_{i j} \quad$ and $j$ in the $x, y, z$, and radial directions

$Z \quad$ acoustic impedance

$z_{i} \quad$ normal distance from wall to molecule $i$

\begin{tabular}{|c|c|}
\hline Greek & \\
\hline$\delta_{T}$ & Tolman length \\
\hline$\dot{\gamma}_{e, a}$ & transition rate \\
\hline$\Delta_{t}$ & $\begin{array}{l}\text { time interval for one time step } \\
\text { of simulation }\end{array}$ \\
\hline$\varepsilon$ & molecular energy component \\
\hline$\varepsilon$ & dielectric function \\
\hline$\varepsilon_{L J}$ & $\begin{array}{l}\text { Lennard-Jones energy } \\
\text { parameter }\end{array}$ \\
\hline$\varepsilon_{\text {rot }}$ & molecular rotational energy \\
\hline$\varepsilon_{w}$ & $\begin{array}{l}\text { wall potential energy } \\
\text { parameter }\end{array}$ \\
\hline$\eta_{e-p h}$ & quantum efficiency \\
\hline$\kappa_{S P}$ & $\begin{array}{l}\text { wavevector of the surface } \\
\text { plasmon }\end{array}$ \\
\hline$\lambda$ & wavelength \\
\hline$\lambda_{\infty}$ & $\begin{array}{l}\text { molecular mean free path in } \\
\text { far ambient }\end{array}$ \\
\hline$\mu_{e}$ & transition dipole moment \\
\hline$\mu$ & chemical potential \\
\hline$\xi$ & molecular degrees of freedom \\
\hline$\rho$ & molecular number density \\
\hline$\rho_{e}(\mathbf{r})$ & charge density \\
\hline$\rho_{l l}$ & liquid density \\
\hline$\hat{\rho}_{l}$ & liquid molar density \\
\hline$\sigma_{l v}$ & liquid-vapor interfacial tension \\
\hline$\sigma_{L J}$ & $\begin{array}{l}\text { Lennard-Jones length } \\
\text { parameter }\end{array}$ \\
\hline$\sigma_{t}$ & $\begin{array}{l}\text { energy accommodation } \\
\text { coefficient }\end{array}$ \\
\hline$\phi, \phi_{i j}$ & $\begin{array}{l}\text { intermolecular potential } \\
\text { function }\end{array}$ \\
\hline$\phi_{L J}$ & $\begin{array}{l}\text { Lennard-Jones } 6-12 \\
\text { intermolecular potential }\end{array}$ \\
\hline$\phi_{w}$ & wall potential \\
\hline$\psi_{i}, \psi_{f}$ & $\begin{array}{l}\text { initial and final state wave } \\
\text { functions }\end{array}$ \\
\hline
\end{tabular}


atom index for molecule $i$ atom index for molecule $j$ corresponding to the Debye model corresponding to the liquid droplet surface corresponding to particles emitted by the droplet corresponding to the liquidvapor interface index for side 1 or side 2 of an interface, impurity scattering corresponding to particles incident on the droplet index for the phonon polarizations

$l$
$n$, non
$r o t$
$t r$
$v$
$w$
$x, y, z$
$\infty$
0
1
2
4

liquid noncondensable rotational translation vapor water in the $x, y$, or $z$ direction, respectively corresponding to far ambient conditions corresponding to conditions in the gas at the droplet surface corresponding to side 1 of an interface corresponding to side 2 of an interface corresponding to $r / r_{d}=4$

\section{DEVELOPMENT OF MD AND DSMC FLUID SIMULATIONS AS TOOLS IN NANOSCALE AND MICROSCALE THERMOPHYSICAL ENGINEERING}

\section{Introduction}

In many nanoscale and microscale fluid systems of interest, molecular dynamics (MD) and direct simulation Monte Carlo (DSMC) modeling can provide valuable insight into the thermophysics and transport mechanisms in the system. MD simulation and DSMC models each have a basic framework that is relatively simple and physically realistic: molecules or particles move through space and interact with other molecules or particles within a spatial domain that is a model of the space in a system of interest. In DSMC models, each particle represents a fixed number of molecules in a gas. For gases, DSMC models thus have the advantage that they can model larger systems with larger numbers of molecules with less computational effort than an MD simulation. For each of these simulation types, submodel elements must be added to the basic framework to completely model the physics. The accuracy of the overall model is critically dependent on the accuracy of these submodels, and efforts to improve these schemes have often focused on improving the accuracy of the submodels.

This segment of this review will explore the issues associated with improving submodels for each of these simulation types. It will first consider examples of each type of simulation, focusing on one in each case that exemplifies advanced modeling of a complex system. This will provide insight into how MD simulations and DSMC have been used in recent studies of the thermophysics of nanoscale and microscale fluid systems and provide the basis for a more concrete discussion of issues associated with development of improved submodels for these simulation schemes. The subsequent discussion will focus on how the submodels affect the accuracy of the simulations and explore how they might be improved.

\section{Simulations of Liquid Films}

In deterministic molecular dynamics simulations, different boundary conditions can be imposed to simulate different system physical constraints. The simplest and 
most common type of MD simulation is the so-called $N V E$ simulation, which considers molecules in a system with fixed total energy $E$, fixed number of molecules $N$, and fixed volume $V$. Alternate strategies have also been developed to model systems held at constant pressure, constant temperature, and/or constant chemical potential (free mass exchange with a surrounding reservoir). Simulations with all three of these constraints are sometimes referred to as $\mu P T$ simulations. Molecules in the simulation are allowed to move in accordance with Newton's laws of motion, as dictated by the influence of intermolecular forces and subject to the system constraints. Positions, velocities, orientations, and angular velocities for each molecule are tracked over the duration of the simulation. Equilibrium macroscopic properties are calculated by averaging the appropriate function of molecular positions and velocities over time. Readers unfamiliar with the fundamental aspects of molecular dynamic simulation methods may wish to consult the references by Hoover et al. [1], Hoover, [2], Haile [3], and Frenkel and Smit [4].

MD simulation studies are particularly useful for exploring the thermophysics of the interfacial region between bulk liquid and bulk vapor phases. MD simulations of interfacial region thermophysics have often modeled a free liquid film. Studies of a free liquid film usually considered the computational domain shown in Figure 1.

The model system for the free liquid film is a liquid layer bounded in the $z$ direction by vapor regions, as shown in Figure 1 . The system is periodic in the $x$, $y$, and $z$ directions. The periodicity requires that any molecule that leaves simulation region on one bounding surface reenters across the opposite face. Early simulations of this type often used the simple radially symmetric Lennard-Jones 6-12 interaction potential model defined by the equation

$$
\phi_{L J}(r)=4 \varepsilon_{L J}\left[\left(r_{0} / r\right)^{12}-\left(r_{0} / r\right)^{6}\right]
$$

For this potential model $\varepsilon_{L J}$ is the depth of the potential well and $r=2^{1 / 6} r_{0}$ is the location of the minimum in the potential well. More recent simulation studies have explored the use of more complex interaction potentials that better model specific molecules. The free liquid film MD simulation has been used to explore the interfacial

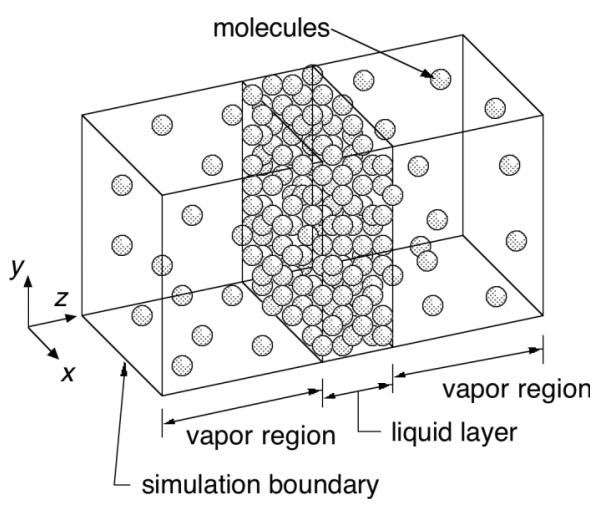

Figure 1. System model used in the simulation of a periodic free liquid film. 
region properties for a variety of fluids, including Lennard-Jones fluids [5], argon [5-8], nitrogen [8], water [9, 10], water and alcohol mixtures [11, 12], hydrocarbons [13], and fluorocarbons [14].

Computations of the surface tension using MD simulation data from the studies with the best interaction potential models have generally produced values that are in fairly good agreement with recommended values based on experimental data. Figure 2 shows the surface tension values for water predicted by the MD simulations of Alejandre et al. [9], which generally agree well with tabulated values recommended by the ASHRAE Fundamentals Handbook [15]. The good agreement indicates that the extended simple point charge (SPC/E) interaction potential for water molecules can yield good results in water MD simulations. Other successful efforts to predict surface tension from MD simulation data for other polyatomic species (i.e., nitrogen [8], hydrocarbons [13], and fluorocarbons [14]) also demonstrate that MD simulation can be a useful predictor of interfacial region thermophysics if it incorporates a physically realistic interaction potential model.

A related category of MD simulation studies is the subset that models thin liquid films on solid surfaces [16-20]. Here we will examine the hybrid model developed by Wemhoff and Carey [18-20] for a liquid water film on a solid surface in contact with is saturated vapor. The simulation domain for these models is shown in Figure 3. The hybrid technique used by Wemhoff and Carey [18-20] combines a deterministic MD simulation of the liquid and vapor regions with stochastic treatment of the far-field vapor region boundary. In this simulation scheme, the imposed far-field pressure may be held constant or iterated as the simulation is advanced in time until the mass in the system stabilizes at the specified temperature. The latter iterative method establishes the equilibrium saturation vapor pressure for the specified temperature, as dictated by the intermolecular force interaction models for fluid-fluid molecular interaction and

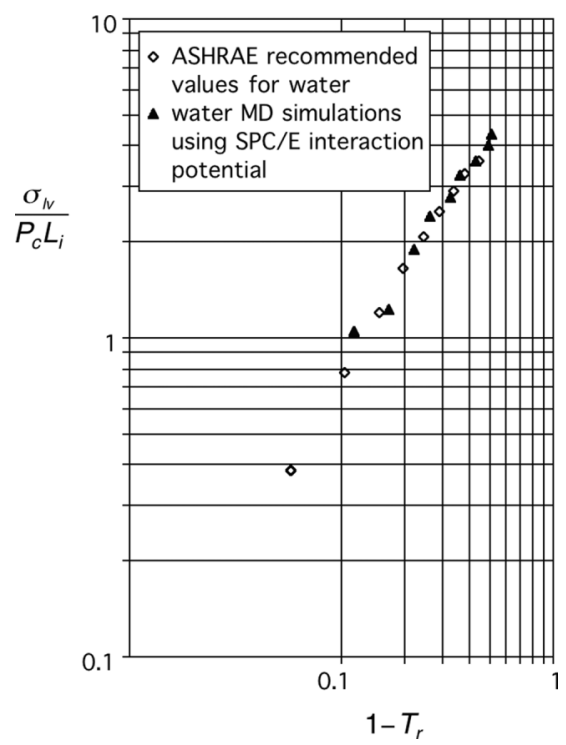

Figure 2. Comparison of MD simulation predictions of surface tension obtained by Alejandre et al. [9] with recommended values from the ASHRAE Fundamentals Handbook [15]. 


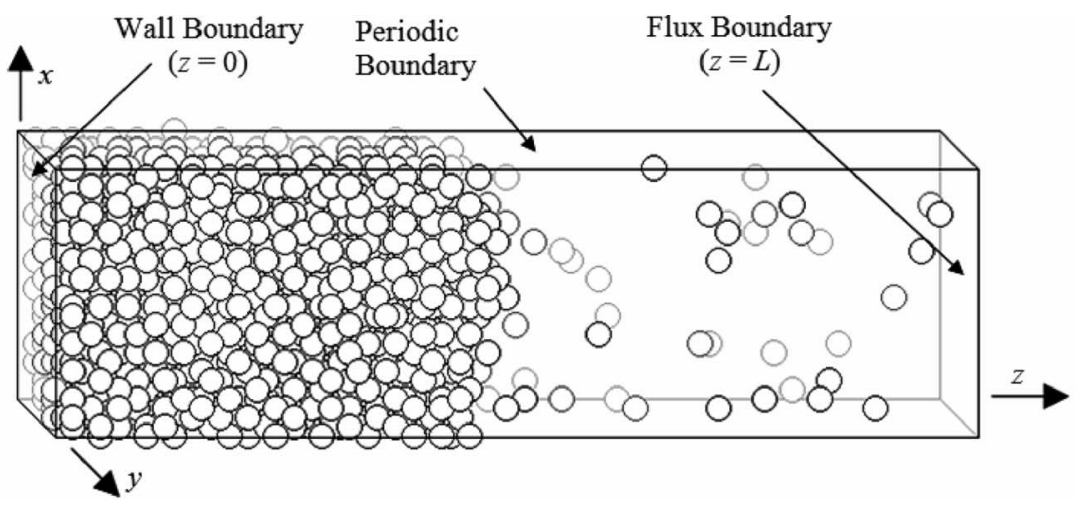

Figure 3. MD simulation model system for a liquid wall film.

interaction between fluid molecules and atoms in the solid surface. These investigators used this approach to model liquid films of simple monatomic species, nonpolar polyatomic molecules, and water. To facilitate a discussion of this type of simulation, its features will be briefly described here.

In the simulations of Wemhoff and Carey [18-20], the positions of the molecules were initialized in a face-centered-cubic (fcc) liquid and vapor lattice structures where the lattice densities correspond to the ASHRAE [15] recommended equilibrium bulk density values for the desired system equilibrium temperature. The molecules in both phases are initially oriented by applying randomly chosen Euler angles via well-known tensor relations [21]. The initial translational velocities and rotational velocities were chosen to match the Maxwell-Boltzmann distribution using the Box and Mueller [22] method.

In these simulations, the system equilibrates by ramping up the system temperature over 30,000 time steps from an initial cool temperature $\left(T_{r}=0.1\right)$ to the desired system temperature by velocity rescaling, where the system temperature is determined at each time step from the kinetic energy by

$$
\sum_{i=1}^{N} \sum_{a=1}^{N_{\text {atom }}} \frac{1}{2} m_{i a} v_{i a}^{2}=K E=\frac{\xi}{2} N k_{B} T
$$

where $m_{i a}$ and $v_{i a}$ are the mass and speed of atom $a$ of molecule $i$, respectively, and $\xi$ is the number of degrees of freedom in each molecule. After 30,000 time steps of velocity rescaling, the system is allowed to settle for an additional 10,000 time steps prior to data collection.

The flux boundary is imposed to preserve mass during the initial 40,000 steps of the simulation, and for the remainder of the simulation the flux of injected molecules follows [18]:

$$
J=\sqrt{\frac{k_{B} T}{2 \pi m}}\left(\frac{P}{k_{B} T}\right)
$$

where $P$ and $T$ are the vapor far field pressure and temperature, $k_{B}$ is the Boltzmann constant, and $m$ is the molecular mass. When a molecule is injected, the $x$ - and $y$-translational velocities are provided via the Box-Mueller technique [22] to match the Maxwell-Boltzmann distributions for molecules entering the domain [18]. 
For interactions between fluid molecules and the wall, the following interaction potential was developed [19]:

$$
\phi_{w, i}\left(z_{i}\right)=\varepsilon_{w}\left[\frac{1}{3}\left(\frac{r_{w}}{z_{i}}\right)^{9}-\left(\frac{r_{w}}{z_{i}}\right)^{3}\right]
$$

where

$$
\varepsilon_{w} r_{w}^{3}=1.5376 \times 10^{-21} \frac{\sigma_{l v}^{1 / 2}}{\rho_{l}}
$$

and

$$
r_{w}=2^{1 / 6} r_{0}
$$

To derive the potential function (4), the model potential for interaction of one fluid molecule with an atom in the surface is integrated to account for the effect of all solid surface atoms on a given fluid molecule [18]. In Eq. (6), the value of $r_{0}$ is a characteristic length in the polyatomic intermolecular potential used for the fluid. In Wemhoff and Carey [18], it is shown that Eq. (4) can be applied upon the fluid molecule at its center of mass. The force upon the center of mass due to the presence of the wall was then translated to the atoms using relations developed by Ciccotti and Ryckaert [23].

In the system containing water molecules and periodic boundaries, the long-ranged electrostatic interaction is generally handled via a three-dimensional Ewald summation technique [24], where a shielding charge distribution is placed on top of the point charges in the simulation to reduce the error of the coulombic potential and force calculations at the cutoff radius. The charge density of the shielding charge converts the dependence of the real-space potential to $\operatorname{erfc}(r) / r$, which decays much more rapidly than the unshielded $(1 / r)$ relationship. In addition, a compensating charge distribution is added to the simulation to effectively negate the introduction of the shielding charge distribution in the simulation. The periodicity of the long-ranged compensating charge due to the periodic boundary conditions allows for it to be handled by a Fourier analysis over the reciprocal lattice generated from repeating the simulation domain in all directions.

Unfortunately, the hybrid system shown in Figure 3 does not feature periodic boundary conditions in the $z$ direction, so the conventional three-dimensional Ewald summation technique cannot be used. Therefore, the two-dimensional periodicity is established using a modified version of the corrected three-dimensional Ewald summation as described by Yeh and Berkowitz [24], where a correction term is added to the three-dimensional Ewald summation for water molecules [25-27] to obtain the relation for the system energy:

$$
U=\frac{1}{4 \pi \varepsilon_{0}}\left\{\begin{array}{l}
\frac{2 \pi}{V} \sum_{\mathbf{k} \neq \mathbf{0}} Q(k)|S(\mathbf{k})|^{2}+\sum_{i=1}^{N} \sum_{a=1}^{N_{\text {atom }}} \sum_{j>i}^{N} \sum_{b=1}^{N} \frac{N_{\text {atom }}}{r_{i a} q_{j b}} \operatorname{erf} c\left(\kappa r_{\text {iajb }}\right) \\
-\frac{\kappa}{\sqrt{\pi}} \sum_{i=1}^{N} \sum_{a=1}^{N_{\text {atom }}} q_{i a}^{2}-\frac{1}{2} \sum_{i=1}^{N} \sum_{a=1}^{N_{\text {atom }}} \frac{q_{i a} q_{j b}}{r_{\text {iaib }}} \operatorname{erf}\left(\kappa r_{i a i b}\right)-\frac{2 \pi}{V} \sum_{i=1}^{N} \sum_{a=1}^{N_{a}} q_{i a} z_{i a}
\end{array}\right\}
$$


where

$$
\begin{gathered}
\mathbf{k}=\frac{2 \pi}{L_{x}} \hat{\mathbf{x}}+\frac{2 \pi}{L_{y}} \hat{\mathbf{y}}+\frac{2 \pi}{L_{z}} \hat{\mathbf{z}} \\
S(\mathbf{k})=\sum_{i=1}^{N} \sum_{a=1}^{N_{a}} q_{i a} \exp \left(i \mathbf{k} \bullet \mathbf{r}_{i a}\right)
\end{gathered}
$$

and

$$
Q(k)=\frac{1}{k^{2}} \exp \left(-\frac{k^{2}}{4 \kappa^{2}}\right)
$$

In the above equations, $q_{i a}, z_{i a}$, and $\mathbf{r}_{i a}$ are the point charge, $z$-position, and position vector of atom $a$ of molecule $i, k$ is equal to $|\mathbf{k}|$, and $\kappa$ is $5.6 / L_{x}=5.6 / L_{y}$. The justification for the corrected three-dimensional Ewald summation is discussed in Wemhoff and Carey [19], as is the justification to extend the original analysis [24] to liquid-vapor systems far from the critical point. In this study, the vapor density is much less than the liquid density, so the corrected 3-D Ewald summation technique provides a reasonable method to predict equilibrium properties of a thin water film on a solid surface.

Although Wemhoff and Carey [20] did not explore the thermal conductivity in their simulation study, the thermal conductivity can be determined from statistics generated by the MD simulation. The method used is commonly referred to as a Green-Kubo relation [28-30], and the formula used for the directional thermal conductivity in the $z$ direction is

$$
k_{t, z}=\frac{1}{V k_{B} T^{2}} \int_{\tau=0}^{\infty}\left\langle J_{z}(0) J_{z}(\tau)\right\rangle d \tau
$$

where

$$
J_{z}=\frac{d}{d t} \sum_{i=1}^{N} z_{i} \frac{1}{2}\left[m_{i} v_{i}^{2}+\sum_{j \neq i}^{N} \phi\left(r_{i j}\right)\right]
$$

In the above relation for $J_{z}, v_{i}$ is the magnitude of the velocity vector of molecule $i, m_{j}$ is the mass of molecule $i$, and $\phi\left(r_{i j}\right)$ is the intermolecular potential function. Directional thermal conductivities in the $x$ and $y$ directions are computed similarly.

In this study, three simulations were performed for water (at $T=100,127$, and $140^{\circ} \mathrm{C}$ ). For the water systems, the molecules were initialized in a $4 \times 4 \times 8$ fcc lattice. The water molecules interacted via the SPC/E potential by Berendsen et al. [31]:

$$
\phi_{i j}=4 \varepsilon_{L J}\left\lfloor\left(\frac{r_{0}}{\left(r_{\mathrm{OO}}\right)_{i j}}\right)^{12}-\left(\frac{r_{0}}{\left(r_{\mathrm{OO}}\right)_{i j}}\right)^{6}\right\rfloor+\frac{1}{4 \pi \varepsilon_{0}} \sum_{a=1}^{3} \sum_{b=1}^{3} \frac{q_{a} q_{b}}{r_{i a b j}}
$$




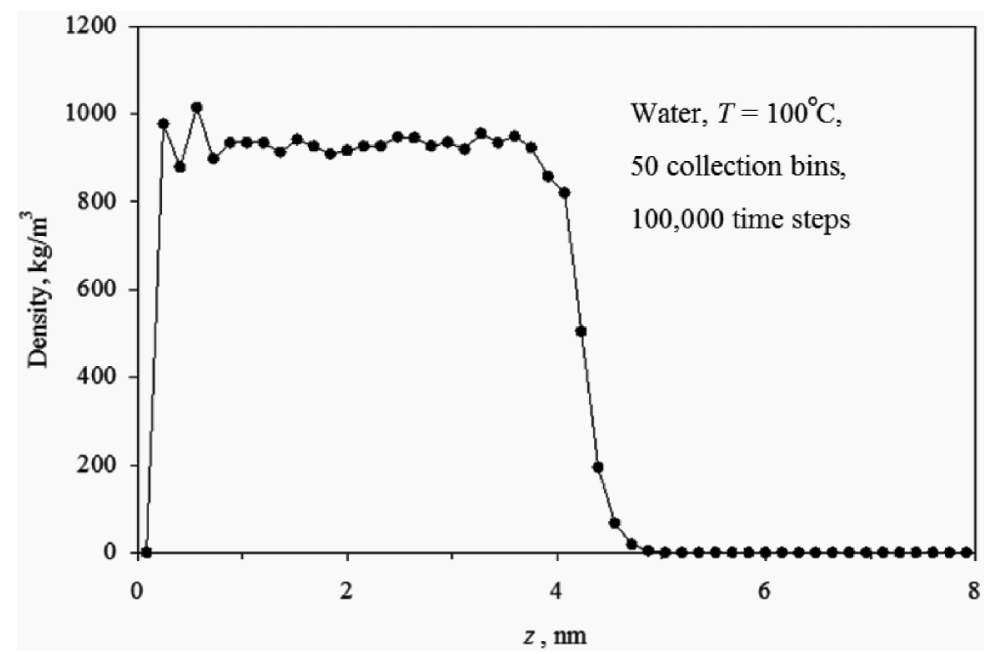

Figure 4. Mean density variation predicted by the MD simulation for a liquid water film [19].

where $\left(r_{\mathrm{OO}}\right)_{i j}$ is the oxygen-oxygen separation distance between molecules $i$ and $j, q$ is the point charge, and $\varepsilon_{0}$ is the permittivity of a vacuum. In the SPC/E potential, the $\mathrm{OH}$ bond length is fixed at $0.100 \mathrm{~nm}$, the $\mathrm{HOH}$ bond angle is $109.47^{\circ}$, and the oxygen atoms contain Lennard-Jones parameters $r_{L J}$ and $\varepsilon_{L J}$ with values of $0.3166 \mathrm{~nm}$ and $78.21 k_{B} \mathrm{~K}$, respectively. The oxygen and hydrogen point charges $q_{O}$ and $q_{H}$ are $-0.8476 e$ and $+0.4238 e$, respectively, where $e$ is the charge of an electron. The application of the point charges in the system was performed using the corrected 3-D Ewald summation technique and is described by Eq. (7). The value of $r_{w}$ was found to be $0.277 \mathrm{~nm}$ from Eq. (6), and the value of $\xi$ in Eq. (2) was 6.

During the simulations, the rigidity of the polyatomic molecules was maintained by the RATTLE algorithm [32]. The water simulations used a time step of $0.5 \mathrm{fs}$. Upon equilibration, water density data in the film were collected by evenly dividing the simulation domain into 50 bins in the $z$ direction. The resulting mean density profile in the film for a film in equilibrium at $100^{\circ} \mathrm{C}$ is shown in Figure 4 . The surface tension was computed from the simulation statistics using the free energy integration method described in Wemhoff and Carey [18]. Figure 5 shows a comparison of surface tension values predicted for the water film interface by the MD simulation with the trend in recommended water surface tension values from the ASHRAE Fundamentals Handbook [15].

Despite the thinness of the liquid film and the attractive forces of the wall molecules, the interfacial tension predicted by the simulation is in fairly good agreement with recommended value of surface tension from the ASHRAE Fundamentals Handbook [15], indicating that wall effects on surface tension for liquid water films are negligible down to thicknesses as small as $4 \mathrm{~nm}$ for this range of temperature.

\section{DSMC Models of Postnucleation Growth of Water Droplets}

As discussed above, DSMC models have the advantage that each particle can model a specified number of molecules. This makes it possible for this type of scheme to be used as a mesoscale model for systems that are too small for continuum models 


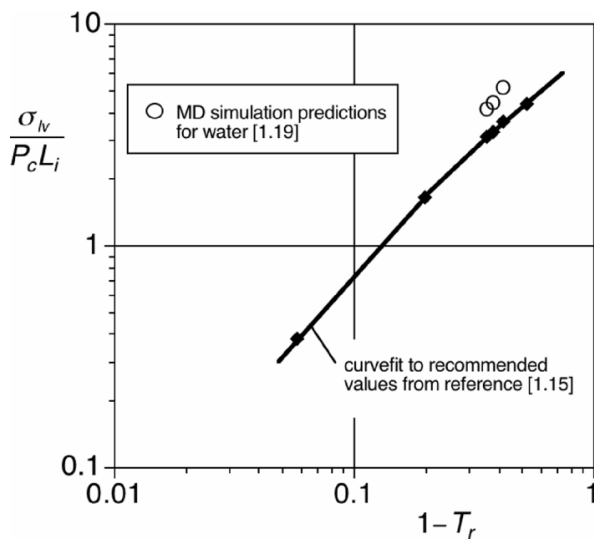

Figure 5. Comparison of surface tension values predicted for the water-film interface by the MD simulation [19] with the trend in recommended water surface tension values from the ASHRAE Fundamentals Handbook [15].

but large compared to molecular dimensions. DSMC models have been used to predict transport in a variety of microscale systems (see, for example, the models described in the literature [33-40]). The discussion here will focus on one specific model type. The DSMC model developed by Carey et al. [38-40] for postnucleation growth of liquid droplets in supersaturated vapor-gas mixture will be considered as an example of a model developed for a particularly complex microscale system.

This model was developed for conditions that match postnucleation droplet growth experiments conducted by Peters and Paikert [41]. The growth conditions are such that conduction in the droplet is fast compared to transport in the gas, and the droplet grows slowly enough that a quasistatic growth mode is a good idealization. This line of reasoning implies that the temperature is essentially uniform within the droplet but may vary slowly with time as the droplet grows. The quasi-equilibrium model treats the radius as fixed during the simulation, which is a good approximation if the characteristic time of the droplet growth process $\left(r_{d} /\left(d r_{d} / d t\right)\right)$ is large compared with the characteristic time for transport in the gas. This requirement is met for the experimental system of interest. The droplet size changes significantly over milliseconds while times on the order of microseconds are required to establish steady transport in the gas.

In the very early stages of postnucleation growth of liquid microdroplets in a supersaturated surrounding vapor or gas mixture, three microscale factors associated with the size of the droplet affect its growth. The first of these is that the droplet size is comparable to or smaller than the mean free path of the molecules in the surrounding gas. For such circumstances, the Knudsen layer region within about one mean free path of the droplet surface is essentially a collision-free zone. Immediately after nucleation, the droplet size may be so small that the extent of the Knudsen layer is large compared to the droplet and the transport is in the free-molecular regime. As the droplet grows, the size of the droplet becomes comparable to and then larger than the thickness of the Knudsen layer with the associated changes from free molecular transport to the transition regime and then to continuum transport. 
The second factor is that for very small droplets, the surface tension shifts the equilibrium vapor pressure from the flat interface value. Classical thermodynamics dictates that this shift is given by the relation

$$
P_{v e}=P_{s a t}\left(T_{d}\right) \exp \left\{\frac{\left[P_{v e}-P_{s a t}\left(T_{d}\right)\right]}{\hat{\rho}_{l} R T_{d}}+\frac{r_{\sigma}}{r_{d e}}\right\}
$$

where

$$
r_{\sigma}=\frac{2 \sigma_{l v}}{\hat{\rho}_{l} R T_{d}}
$$

As will be seen in equations presented later, the vapor pressure is a key factor in predicting the molecular flux from at interface. Equation (14) also implies that for a specified vapor pressure, the equilibrium saturation temperature will be shifted at small droplet radii. This shift will also directly affect the driving potential for heat transfer at the interface.

The third factor affecting droplet growth is that for extremely small droplets, the interfacial tension may be a function of the droplet radius. Molecular thermodynamic analysis suggests that the interfacial tension will vary with radius of curvature when the radius of curvature becomes comparable to the thickness of the interfacial region (see Tolman [42]). From this line of analysis it can be argued that for small droplets or bubbles, the surface tension varies with radius of curvature according to a relation of the form

$$
\sigma_{l v}=\sigma_{l v, \infty}\left(1-\frac{2 \delta_{T}}{r_{i}}\right)
$$

where $\sigma /_{v, \infty}$ is the interfacial tension for a flat interface $\left(r_{i} \rightarrow \infty\right)$ and $\delta$ is the so-called Tolman length scale, below which the radius of curvature affects the magnitude of the interfacial tension. This effect can be important, for example, during postnucleation growth of embryonic bubbles or droplets that are just larger than critical size.

The particle simulation method used by Carey [40] to model transport in the gas mixture surrounding the droplet was a derivative of the direct simulation Monte-Carlo technique pioneered by Bird [41]. Some of the refinements developed by McDonald [43] and Baganoff and McDonald [44] were also incorporated into the scheme. The details of the particle simulation method used are described in Carey [40] and Bird [41]. Some of the features relevant to modeling microscale effects will be summarized here. To minimize memory requirements and computational time, the simulation domain was limited to one octant around the droplet, as shown in Figure 6. In addition, to avoid the computational costs of extending the simulation out to large radial distances, in these studies the simulation domain was limited to a radial distance of four droplet radii. For free molecular conditions, the simulation boundary condition was set to the ambient conditions at $r=4 r_{d}$. For transition regime conditions, the simulation boundary condition was matched to the continuum transport solution beyond $r=4 r_{d}$.

For transition regime conditions (moderate $K n_{D}$ ), the temperature and water vapor concentration gradients decrease rapidly with distance from the droplet, approximately proportional to $r^{-2}$. Consequently, while the temperature may drop 


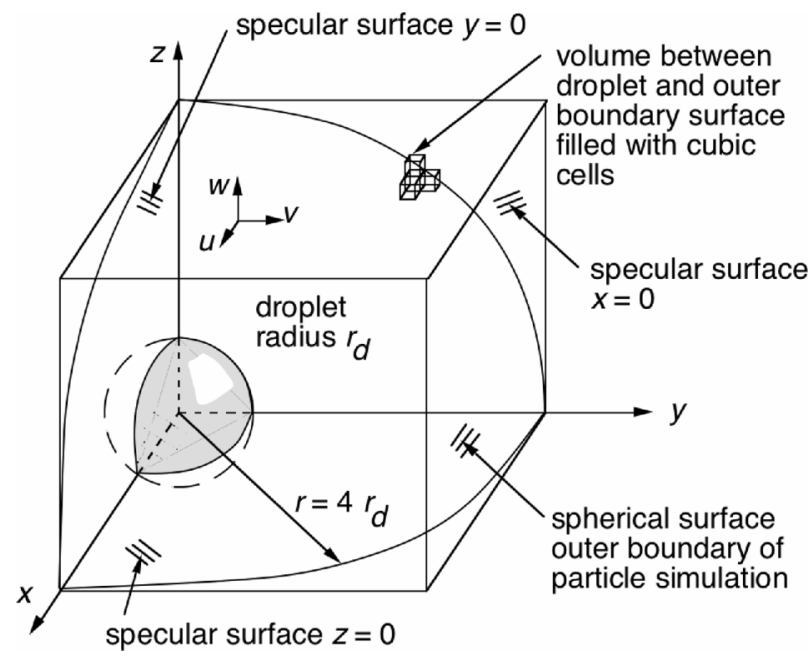

Figure 6. Computational domain for simulation used by Carey et al. [40].

and the water concentration may rise significantly over a few mean free paths near the sphere, beyond $r=4 r_{d}$ it will take many free paths for the temperature to change significantly. The local Knudsen number $\lambda(d T / d r) /\left(T_{d}-T_{\infty}\right)$ decreases rapidly with distance from the sphere, causing the temperature and concentration profiles to approach those for continuum transport at large $r$. This line of reasoning, which divides the region near the sphere into a noncontinuum region close to the body and a continuum transport region farther away, is the basis for the matching procedure used at $r=4 r_{d}$.

The octant simulation region shown in Figure 6 was bounded by the surface of the sphere at $r=r_{d}$, the outer boundary at $r=4 r_{d}$, and specularly reflecting planar surfaces at $x=0, y=0$, and $z=0$. The specular surfaces that lie along planes of symmetry have the effect of giving the octant the same statistical behavior as a complete sphere surrounded by particles. Each particle in the simulation represents $N_{m p p}$ molecules. The region bounded by these surfaces was filled with cubic cells that were used to collect collision candidate pairs and define regions over which particle properties were averaged to obtain macroscopic thermodynamic characteristics of the gas adjacent to the droplet.

Particles that move beyond the outer boundary when the particles are advanced along their trajectory were simply removed from the simulation. During each time step, particles of each species are added to the simulation just inside the $r=4 r_{d}$ boundary. The rate of water particle addition $n_{w}^{Y}$ and the rate of nitrogen particle addition $n_{n}^{Y}$ are computed as

$$
\begin{aligned}
& n_{w}^{\dot{Y}}=N_{m p p}(\pi / 2)\left(4 r_{d}\right)^{2} j_{w} \\
& n_{n}^{\dot{Y}}=N_{m p p}(\pi / 2)\left(4 r_{d}\right)^{2} j_{n}
\end{aligned}
$$


where $j_{w}$ and $j_{n}$ are the fluxes of water and nitrogen molecules across a surface predicted by kinetic theory for a mixture at a pressure $P_{\infty}$, temperature $T_{4}$ and water mole fraction of $X_{w, 4}$.

$$
\begin{gathered}
j_{w}=\left(\frac{k_{B} T_{4}}{2 \pi m_{w}}\right)^{1 / 2}\left(\frac{X_{w, 4} P_{\infty}}{k_{B} T_{4}}\right) \\
j_{n}=\left(\frac{k_{B} T_{4}}{2 \pi m_{n}}\right)^{1 / 2}\left(\frac{\left(1-X_{w, 4}\right) P_{\infty}}{k_{B} T_{4}}\right)
\end{gathered}
$$

This treatment allows the simulation domain to establish whatever mean particle density is necessary to balance the inward and outward fluxes at the outer boundary.

As noted earlier, when molecules from a surrounding gas enter the interfacial region near a solid or liquid phase, experimental evidence and molecular simulation studies indicate that only a fraction of the incident molecules thermally interact with the molecules of the condensed phase. In the DSMC models of Carey et al. [38-40], this behavior is stochastically represented by specifying an accommodation coefficient $\sigma_{t}$ that is taken to equal the fraction of incident molecules that thermally interact with the droplet surface.

If a particle strikes the droplet surface during a given time step, a random number $\Re$ uniformly distributed on $[0,1]$ is compared to the specified accommodation coefficient. If $\Re>\sigma_{t}$, the particle is diffusely scattered from the surface without interacting thermally. Otherwise, it merges into the liquid droplet, delivering its energy, including an energy of vaporization, to the droplet. Computationally, particles that merge into the droplet are removed from the simulation. The number of particles absorbed in this manner is counted, and the energy delivered to the droplet by these particles is summed.

In a subsequent portion of the time step calculations, particles are added to the simulation domain just beyond $r=r_{d}$. Before executing the emission of particles from the droplet surface, the temperature of the droplet surface is computed. Kinetic theory predicts that for an interface at equilibrium, the flux of emitted water vapor molecules is given by

$$
j_{w}=\sigma_{t} \frac{P_{v e}\left(T_{d}\right)}{\left(2 \pi m_{w} k_{B} T_{d}\right)^{1 / 2}}
$$

For the spherical microdroplets considered here, the equilibrium vapor pressure $P_{v e}$ in the above relation is a function of the droplet temperature $T_{d}$ and the droplet radius $r_{d}$ as given by Eq. (14). In the limit of long times, the steady-state energy exchange at the interface must satisfy conservation of energy. Carey et al. [38-40] combined Eqs. (14) and (21) and used the fact that $P_{v e}-P_{s a t}\left(T_{d}\right) \ll 2 \sigma_{l v} / r_{d e}$ to obtain the following form of the conservation of energy relation:

$$
\begin{aligned}
\sum_{i=1}^{N_{\text {steps }}}\left(E_{\text {gain }}\right)_{i}= & 3 k_{B} T_{d} N_{\text {mpp }} \sum_{i=1}^{N_{\text {steps }}}\left(N_{\text {non }}\right)_{i} \\
& +\frac{\pi r_{d}^{2} \sigma_{t} P_{\text {sat }}\left(T_{d}\right)}{2\left(2 \pi m_{w} k_{B} T_{d}\right)^{1 / 2}} \Delta t\left(\hat{u}_{l v}+\frac{1}{2} k_{B} T_{d}\right) \exp \left(\frac{2 \sigma_{l v}}{\rho_{l} r_{d} R T_{d}}\right)
\end{aligned}
$$


In the above equation, $\left(N_{\text {non }}\right)_{i}$ is the number of noncondensable molecules that thermally interact with the surface and then are reemitted into the gas and $\left(E_{\text {gain }}\right)_{i}$ is the energy delivered by molecules colliding with the droplet surface in time step $i$. The left side of the above equation is the total energy delivered by molecules colliding with the surface through Nsteps time steps of the simulation. The right side is the energy carried away by molecules leaving the droplet surface. The first term on the right represents energy carried away by noncondensable gas molecules reemitted to the gas, and the second represents energy removed by water molecules.

Use of Eqs. (14), (21), and (22) in the model requires a means of evaluating the interfacial tension that accounts for possible dependency on droplet radius. For water, Pruppacher and Klett [45] derived a relation for surface tension dependency on droplet radius that can be cast in the form that is equivalent to Eq. (16) with $\delta_{T}$ set to a recommended value of $0.157 \mathrm{~nm}$. This relation was used in this investigation, with the flat-interface interfacial tension $\sigma_{l v, \infty}$ determined from

$$
\sigma_{l v, \infty}=0.07583-0.0001477\left(T_{d}-273.2\right)
$$

where $T_{d}$ is in $\mathrm{K}$ and $\sigma_{l v}$ is in $\mathrm{N} / \mathrm{m}$. This relation is an established surface tension relation for a water liquid-vapor interface at equilibrium (see Carey [40]), which is extrapolated here to nonequilibrium conditions.

It should be noted that combining Eqs. (14) and (16), using the fact that $P_{v e}-$ $P_{s a t}\left(T_{d}\right) \ll 2 \sigma_{l v} / r_{d e}$ and solving for the equilibrium radius, yields

$$
r_{d}=\frac{2 \sigma_{l v, \infty} / \rho_{l} R T_{d}}{\ln \left[P_{v e} / P_{s a t}\left(T_{d}\right)\right]}-2 \delta_{T}
$$

This relation indicates that the equilibrium radius of a droplet, which is the critical radius for a nucleating embryo, is decreased by $2 \delta_{T}$ due to the variation of surface tension with radius. As discussed in the next section, this has an important effect on the early growth after nucleation. For the smallest droplets considered in this study, the interfacial tension is more properly interpreted as the mean interfacial free energy per unit area of the droplet. Its use in this model analysis is consistent with this interpretation.

In Eq. (22) $P_{s a t}$ and $\hat{u}_{l v}$ are functions of temperature. For the purposes of the simulation calculations, Carey et al. [38-40] used curve-fits to recommended property data to account for the temperature variation of these parameters. The droplet temperature was computed at each time step by iteratively solving Eqs. (16), (22), and (23) simultaneously with relations for properties as a function of temperature. A guess of the surface temperature was provided to initiate the simulation. As the number of time steps increases, the surface temperature computed in this manner converges to a value that is interpreted as being the actual physical temperature of the droplet for the quasi-steady process being modeled in the simulation.

Once the surface temperature of the droplet was determined for a given time step, particles were computationally emitted from the droplet surface. First, Nnon noncondensable particles were emitted, so that there is no net transfer of the noncondensable species to the droplet during the time step. Then, particles of the condensable species were emitted from the droplet surface until an energy balance was achieved. 
Energy and mass balances at the interface were used to determine the net heat flux from the droplet surface (carrying away the latent heat of the net condensation effect) and the net mass flux of water vapor at the droplet surface $m_{d}^{\prime \prime}$ (see Carey et al. [38-40]). The growth rate of the droplet was then computed as

$$
\frac{d r_{d}}{d t}=\frac{m_{d}^{\prime \prime}}{\rho_{l}}
$$

In the simulation calculations in these studies, the simulation domain shown in Figure 6 was filled with cubic cells that formed a uniform Cartesian mesh. The radius of the droplet in the simulations ranged from 0.24 to $1000 \mathrm{~nm}$. The effects of cell and time step sizes on the results of the simulation were explored and consequently the cell size was chosen to be on the order of one mean free path. The mean free path for the system considered here is on the order of $90 \mathrm{~nm}$. For the droplet sizes considered, this corresponds to a range of Knudsen number $K n_{D}$ from 0.044 to 186 . The time step was chosen so a particle with average speed traveled approximately half a mean free path in one time step. Generally, the results were found to be insensitive to the choice of these parameters as long as they were close to the values indicated by these guidelines. The side of the cell varied from $0.24 \mathrm{~nm}$ for the smallest droplets to $0.05 \mu \mathrm{m}$ for the largest droplets considered in this study. The number of cells used was a compromise between accuracy and computational effort. The ratio of the number of molecules per particle $N_{m p p}$ was chosen in each simulation to compromise between computational accuracy and storage limitations on the computer. The values used in the calculations reported here ranged from $6.5 \times 10^{-6}$ to 1784 molecules per particle. Simulations were typically run for 16,000 to 800,000 time steps, with a time step typically being $20-200$ ps.

For an accommodation coefficient value of 1.0, the predictions of this type of simulation were found to agree well with droplet growth rates inferred from measurements in a supersaturated mixture of argon and water vapor for droplet radii greater than $0.4 \mu \mathrm{m}$ [38]. The study by Carey [40] considered droplet radius values at or below the critical radius and included the model described above to account for the variation of surface tension with radius of curvature.

Carey et al. [38] found that simulation calculations for $\sigma_{t}=1.0$ best fit the droplet growth rate data from the experiment of Peters and Paikert [46]. Two values of the accommodation coefficient, 1.0 and 0.6 , were therefore used in the simulation calculations in subsequent studies. The simulation calculations in these studies were performed for one of the argon-water vapor mixtures tested experimentally by Peters and Paikert [46]. These calculations span the range of droplet radius from values near the critical radius $r_{c}$ where nucleation occurs to $1.0 \mu \mathrm{m}$ where transition conditions merge into the continuum transport regime.

These DMSC simulations provided detailed information about the temperature and concentration fields near the embryo droplets. An example of the predicted fields for a droplet with a radius of $20 \mathrm{~nm}$ is shown in Figure 7. For this case the Knudsen number is high, corresponding to free molecular transport, and the temperature field is flat, with noticeable temperature slip near the interface.

The simulation prediction of the variation of droplet growth rate with droplet size for one of the argon-water vapor mixtures studied by Peters and Paikert [46] is 


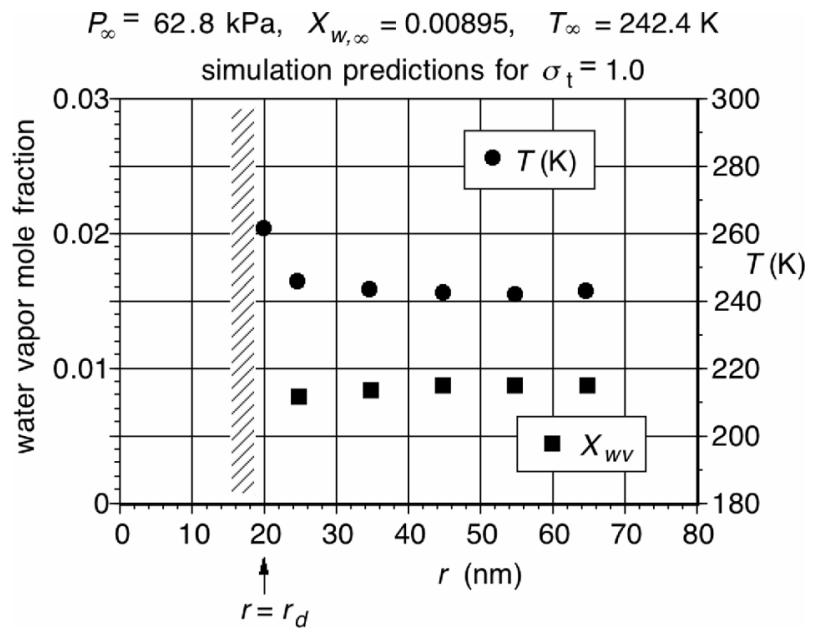

Figure 7. Temperature and concentration fields predicted by the DSMC model of Carey [39] for a water droplet with a radius of $20 \mathrm{~nm}$.

plotted in Figure 8. Results are shown for droplet radii ranging from just above the critical radius (for homogeneous nucleation, see Carey [47]) to droplets with a radius of $1 \mu \mathrm{m}$. Simulation results are shown with the effect of surface tension included and with it artificially removed ( $\sigma_{l v}$ set to zero). For both sets of predictions in this figure, the Tolman length was set to zero so no effect of radius on interfacial tension is included. It can be seen that these predictions of the growth rate variation with radius are the same except at very low radii. The surface tension effect on equilibrium vapor

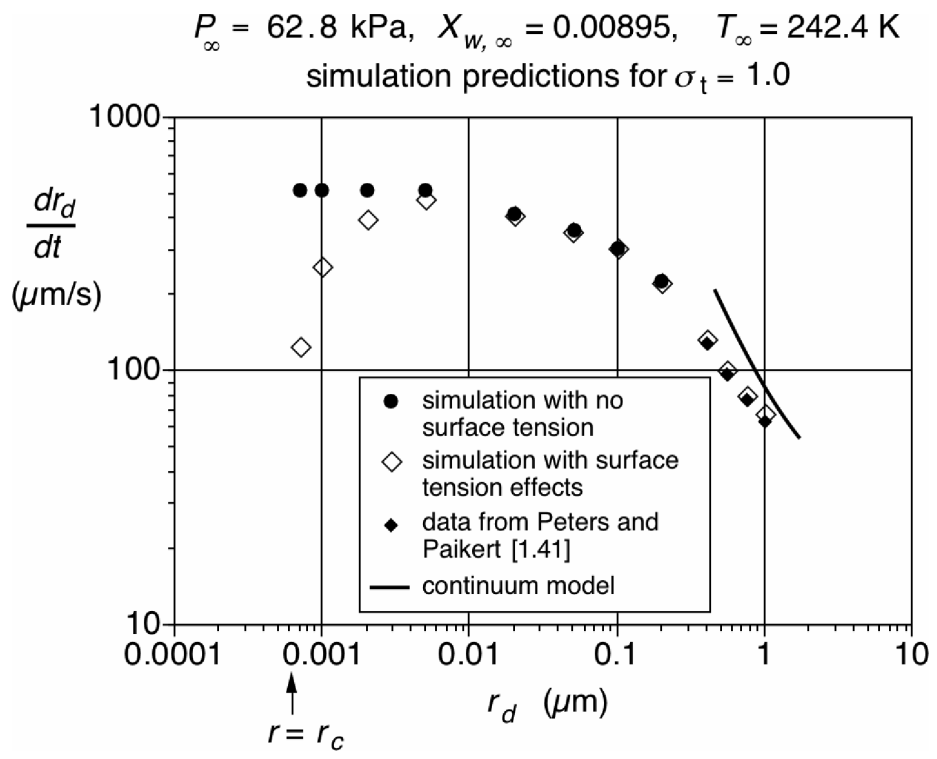

Figure 8. Comparison of droplet growth rates for conditions in the experiments of Peters and Paikert [41]. 
pressure results in a strong reduction in the droplet growth rate as the critical radius is approached. Inclusion of the radius effect on surface tension shifts the growth rate points slightly to the left for $r$ below $2 \mathrm{~nm}$ in Figure 8 [40]. Also shown in Figure 8 is the prediction of standard continuum theory using Fourier's law and Fick's law to predict the simultaneous heat and mass transfer associated with quasi-steady condensation at the indicated conditions. The governing equations and boundary conditions and the method used to solve them are described in Carey [39] and are not repeated here.

Of particular interest is that the simulation model predicts that the droplet growth rate achieves a maximum value in the transition regime, which is attributable to the interaction of surface tension effects and transport variation with droplet size. For the small droplet sizes characteristic of conditions immediately after onset of nucleation, the heat transfer coefficient is large, but the driving temperature difference is small because of the surface tension-induced vapor pressure shift at small droplet sizes. As the droplet grows in size, the vapor pressure shift diminishes and the driving temperature difference increases faster than the heat transfer coefficient decreases. This initially produces an increase in the growth rate as the droplet grows. Eventually the reduction in the heat transfer coefficient dominates, causing the droplet growth rate to peak and then decline with increasing droplet size. It is noteworthy that these results are consistent with the predictions of the kinetic theory model of Peters and Meyer [48], which also predicts a maximum in the droplet growth rate with increasing size for water droplets growing in supersaturated pure steam.

The model for this specific system demonstrates how several microscale mechanisms that affect this system interact to produce the variation in droplet growth rate indicated in Figure 8. As seen in Figure 8, the continuum model predicts a progressively increasing droplet growth rate as the droplet radius decreases. When noncontinuum (high $K n_{D}$ ) effects are included, the growth rate asymptotically approaches a constant as the radius approaches zero as the transport across the Knudsen layer near the interface approaches the ballistic limit.

At extremely small droplet radius values, the interfacial tension alters the thermodynamic equilibrium conditions in a manner that reduces the droplet temperature established during the quasi-equilibrium growth process. This reduces the driving potential for heat transfer from the droplet, thereby reducing the growth rate. As the critical radius is approached, the droplet growth rate tends toward zero. If a droplet radius less than the critical radius is specified for the simulation, the predicted growth rate is negative. These predictions are consistent with the thermodynamic prediction that droplets of less than critical size will spontaneously shrink, whereas those of greater than critical size will spontaneously grow. Inclusion of the model for the dependence of surface tension on droplet radius slightly shifts the interface equilibrium condition at radii near the critical radius. Consistent with Eq. (24) and the results shown in Figure 8, the variation of the interfacial tension with radius in this model shifts the critical radius for the onset of nucleation to a slightly lower radius. Accurate prediction of the near-interface transport during this type of droplet growth clearly requires a model that incorporates treatments of the multiple microscale effects involved and correctly treats interactions among those mechanisms. 


\section{Discussion}

The above examples illustrate how submodels are incorporated into the basic frameworks of MD simulation and DSMC schemes. For each of these model types, three categories of submodels must be provided to construct a complete simulation. The categories, and the challenges for improving the submodels, are discussed below.

\section{Simulation Submodels}

Potential functions for molecular interactions Because the forces between molecules are determined from the gradient of the potential function, this submodel is centrally important to the accuracy of the simulation. Early MD investigations were often based on the Lennard-Jones potential interaction model. This model is useful for molecules that exhibit only van der Waals force interactions and are adequately represented by a spherically symmetric potential function. The Lennard-Jones potential, and other radially symmetric potentials, generally do not work well for polyatomic molecules that lack spherical symmetry and polar molecules (having a permanent dipole moment). Development of a potential function model that accurately treats the combined dispersion (van der Waals) and dipole force interactions between water molecules continues to be a subject of current research. The MD model of Wemhofff and Carey [19] for a water film on a solid surface discussed above uses the simple point charge extended model (SPC/E). As noted by Ohara [49], there is no potential model that precisely reproduces all the characteristics of water. Ohara [49] also observed that the SPC/E is arguably the most successful potential model, in terms of its simple mathematical formulation and its ability to predict density and surface tension for water using it in MD simulations.

The SPC/E and other potential models that incorporate coulombic potential effects between charges have the problem that the coulombic potential dies off slowly with distance, requiring long cutoff distances for interactions with a given molecule. Use of long cutoff distances requires more computation since a given molecule will interact with more molecules. In periodic systems, like the periodic free film domain in Figure 1, the Ewald summation technique can be used to reduce the computational load. However, when a system is not symmetric, this method cannot be employed. Wemhoff and Carey [19] modified the 3-D Ewald summation method to their system with 2-D symmetry to deal with this issue. The nonspherical character, hydrogen bonding effects, and the polar character of molecules result in increasing complexity of the potential interaction for systems with larger organic molecules, alcohols (which can be large and polar), and in water solutions with ions. Development of accurate and computationally efficient intermolecular potential functions for these more complex circumstances remains one of the primary challenges in efforts to construct MD simulations that can be used to model thermophysics and heat transfer in microscale and nanoscale fluid systems.

Domain boundary conditions The molecular behavior at the boundaries of the simulation domain must be handled in a way that is consistent with the nature of the physical system being modeled. Despite the rapidly increasing power of computing platforms for MD simulations, the number of molecules that can be handled with a reasonable computation time in a simulation is limited. Periodic domains for the simulation provide a means to model a larger system with a smaller number of molecules. However, in systems like the water liquid film on a solid surface 
discussed above, the domain is not periodic in the direction normal to the solid surface. The hybrid scheme of Wemhoff and Carey [19] uses a stochastic boundary condition to treat the far vapor boundary as if it is in equilibrium with an ambient vapor region at a fixed condition. This provides a physically realistic treatment of the conditions typically imposed on a real liquid film on a solid surface, at the expense of some additional computational effort. Further development of innovative ways of handling domain boundary conditions is needed to extend MD simulation methods to a broader range of physical systems.

Treatment of interaction with solid surfaces This submodel category arises only in simulations in which the molecules in the domain interact with atoms in a solid-phase boundary or internal element. There are two ways that this type of interaction has typically been handled. One is to treat the solid as a collection of fixed atoms, each of which interacts with each fluid molecule in the adjacent fluidfilled region. This effectively treats the solid and fluid regions as a composite MD simulation, which doubles the effort compared to simulating the fluid region alone. The alternative approach is to develop a surface potential function for a semi-infinite solid that accounts for the mean interaction of all the solid atoms with each fluid molecule. This approach has been used by a number of investigators (see Wemhoff and Carey [18] for a summary) and, as shown by Wemhoff and Carey [18] and others, this type of surface potential can be derived by integrating a solid-fluid intermolecular potential function model to account for contribution by all solid atoms. Use of a surface potential function usually compromises accuracy somewhat, with a substantial gain in computing efficiency. Development of strategies to more effectively use this approach would greatly enhance simulations involving fluid interactions with solid surfaces.

\section{DSMC Submodels}

Collision probability distribution functions In DSMC models, probability distribution functions are sampled to stochastically determine the outcome of particle collisions. These functions are therefore centrally important to the accuracy of the model. Use of DSMC models for gas dynamics and vehicle reentry flow modeling in the aerospace field has led to development of probability distribution functions for a variety of common gas species. Results from these areas can be adapted to nano- and microscale system modeling. This, in fact, was done in the studies of Carey et al. [33, 37-40], which involved only simple molecules such as nitrogen, water, and argon. However, in systems that contain larger and/or more complex molecules, models of collision probability distribution functions may not have been determined, and development of an appropriate distribution function may be a necessary first step in construction of a DSMC simulation scheme.

Domain boundary conditions The particle behavior at the boundaries of the simulation domain must be handled in a way that is consistent with the nature of the physical system being modeled. In this type of model, particle interactions with boundaries are typically handled stochastically, with a probability distribution derived from a physical model. Most often, the probability distribution is derived from kinetic theory for a gas at equilibrium (i.e., it conforms to Boltzmann distributions of energy storage). If the gas at the boundary is not in equilibrium, determination of probability distribution functions for stochastic treatment of incoming molecules is more difficult. Also, if the gas is dense and attractive forces 
are important, its behavior may deviate from classical kinetic theory. In cases where classical kinetic theory is not accurate, development of probability distributions using alternate treatments is needed.

Treatment of interaction with surfaces This arises in simulations in which the particles in the domain interact with molecules in a liquid- or solid-phase boundary or internal element. For real solid surfaces, molecules that hit the surface may be scattered with little or no interaction, or they may be adsorbed onto the surface for a time interval long enough to reach thermal equilibrium with the solid. After some residence time, it may escape back into the gas phase with energy consist with Boltzmann distributions at the solid surface temperature. Interaction with a solid surface is often modeled with an accommodation coefficient that models the degree to which the interacting particle approaches equilibrium with the surface condition. Operationally, the accommodation coefficient is taken to be the probability that an incident particle interacts, and this probability is used to stochastically determine whether an incident particle interacts or is elastically scattered with no interaction. Particles that interact and reenter the gas are stochastically given translational velocities and rotational energies consistent with kinetic theory and Boltzmann distributions for the surface temperature.

For liquid-gas interfaces, like that considered in the droplet studies of Carey et al. [38-40], the circumstances are more complicated. The liquid emits molecules at a rate dictated by the vapor partial pressure at the droplet temperature, and molecules leaving carry latent heat energy with them. Vapor molecules incident on the interface, if they interact, deposit latent heat energy and merge in to the droplet. Incident noncondensable gas molecules may or may not interact thermally, but either way they are ultimately returned into the gas phase. Carey et al. [38-40] treated these interactions with a constant accommodation coefficient and the assumption that conservation of energy, local thermodynamic equilibrium, and kinetic theory apply at the interface. In the cases considered in these studies, these idealizations appeared to work well. However, in general it is unclear how well these idealizations apply to liquid-vapor interfaces undergoing condensation or evaporation. The assumption of local thermodynamic equilibrium and the accuracy of a constant accommodation coefficient are suspect, since collisions with emitted molecules may be limited near the interface or surface, and the degree of thermal interaction may be affected by the incoming translational energy of the particle. Further exploration of these issues is needed to develop better treatment of surface interactions in DSMC models.

MD simulations and DSMC models show great promise as tools for modeling the thermophysics and heat transfer in nanoscale and microscale systems. However, as described above, their widespread use as tools for analyzing such systems will require improvements to the submodel components. A key aspect of this challenge is to devise submodels that are physically accurate and computationally efficient.

\section{NANOSCALE THERMAL RADIATION}

\section{Introduction}

Thermal radiation has a rich foundation spanning electromagnetic wave propagation, statistical thermodynamics, and quantum mechanics, which was laid down through the works of many scientific giants such as Maxwell, Kirchoff, Rayleigh, 
Wien, Planck [50], and Einstein. Maxwell's electromagnetic wave equations govern the propagation of thermal radiation. Planck's law of blackbody radiation was derived based on the revolutionary concept of energy quantization. Later, Einstein further pointed out the granular, or particle, nature of energy quanta and predicted the existence of the stimulated emission process. These developments eventually led to quantum mechanics that forms the foundation for explaining the microscopic processes of emission and absorption. Treatment of engineering thermal radiation problems is typically based on Planck's blackbody radiation law and the photon Boltzmann transport equation or, equivalently, the equation of radiative transfer. Such treatment ignores the phase information carried by electromagnetic waves and is valid when the characteristic structural length is much longer than the photon wavelength. However, as the characteristic structural size becomes comparable to or smaller than the wavelength, the engineering treatment is no longer valid. In the following, a few topics will be discussed to illustrate the special issues encountered in nanoscale thermal radiation.

\section{Coherent vs. Incoherent Radiative Transfer}

The treatments of engineering thermal radiation problems usually neglect the phase information of photons and consider photons only as particles that experience incoherent transport. Ray tracing or Monte Carlo methods and equations of radiative transfer are used to solve these problems. Such transport is incoherent. To account for the phase information, Maxwell's electromagnetic wave equations need to be solved. Capturing the phase information means that the spatial and temporal steps used in the numerical simulations must be much shorter than the wavelength and the period of photons participating in transport. For macroscopic structures, such simulations are usually not possible, or necessary, because multiple scattering events randomize the wave superposition and the results of full-scale Maxwell equations-based solutions approach those of ray tracing. It is usually recognized that wave approaches based on solving Maxwell's equations are needed when structures are comparable or smaller than the dominant photon wavelength. However, transition from coherent transport to incoherent transport remains to be an area that needs further attention [51].

A radiation source has both spatial and temporal coherence properties. In a radiation source, thermal radiation is emitted through the transition of the elementary energy carriers (electrons, phonons, molecules, etc.) from a higher energy state to a lower energy state. The temporal coherence property of a radiation source is a measure of the correlation of the emission from the same point in the source as a function of time. The spatial coherence property measures the correlation of the emission from different points of the source. It is assumed that each atom in the source emits a wave packet that spans a time interval, $\tau$, and that successive emissions of the atoms are not correlated. This wave packet can be decomposed, using Fourier transforms, as the superposition of a series of monochromatic waves covering a frequency range $\Delta v$ centered at $v_{0}$ - the frequency determined by the energy difference of the matter that the emission occurs. Each of the monochromatic waves can be treated as coherent and thus propagates following the Maxwell equations. The superposition of these waves leads back to the wave packet with a time span of $\tau$. The coherence time is thus roughly related to the spectral width by $\tau \propto 1 / \Delta v$ [52]. The spatial coherence length of the source is determined by whether the emissions from 
two points of the source are spatially related or not. Taking a gaseous source as an example, the emissions of two molecules or atoms at different locations of the source are unlikely to be related. However, we will see later that surface waves in some solid materials are highly correlated and thus thermal emission from such a source can have a high degree of spatial coherence [53].

The transition from coherent to incoherent photon transport is an issue that is of significant interest to different communities, including heat transfer. A few papers have considered the radiative properties of a single layer and double layers of thin films and concluded that the wave effects can be neglected when the coherence length, $\ell_{c}$, is shorter than the film thickness [54]. However, studies of wave propagation in periodic structures have shown that the same conclusion is not applicable to such structures as interference effects lead to the formation of new photonic band structures [55]. It seems that the structures can significantly modify the temporal coherence property of thermal radiation. Structural randomness does not always lead to incoherent photon transport. Other physical processes, such as wave localization, can occur. Localization is a topic of great interest in a wide range of wave propagation problems [56], and its impacts on radiation transfer need further exploration.

\section{Far-Field Thermal Emission from Nanostructures}

In the classic treatment of blackbody radiation, Planck clearly stated "Throughout the following, ... the linear dimension of all parts of space considered, as well as radii of curvature of all surfaces ... are large compared with the wavelengths of the rays considered." He also stated that the emitting object itself is in thermal equilibrium. An interesting question to ask is whether thermal emission from nanostructures can be higher than that of a blackbody at the same temperature.

The answer is yes, although existing examples are scarce. One example is thermal emission from small particles [57]. When the diameter of the particle is comparable to the wavelength, some particles can have an emissivity larger than one. Figure 9 shows an example of calculated emissivity of a spherical particle. Such results can be calculated from the indirect method that calculates first the absorptivity and then equate the latter to the emissivity according to the Kirchoff law or the direct method, where thermal emission is computed directly [58]. The two approaches give consistent results. Other structures such as cylindrical particles can also have emissivity larger than one. Inside solid materials, the blackbody radiation intensity is roughly proportional to the square of the refractive index n. However, in a planar surface, radiation cannot escape the solid due to internal reflection. In micron- and nanometer-sized particles, the nonplanar geometry facilitates the emission of such evanescent waves so that the total emission is higher than that allowed for free space blackbody emission.

Recently, there was some debate on whether thermal emission from photonic crystals can exceed that of blackbody radiation [59]. Photonic crystals are periodic arrangements of structures that mimic periodic arrangements of atoms in a crystal and can exhibit band structures similar to that of electrons and phonons in solid crystals [60]. A simple example is a Bragg reflector that is made of a quarter wavelength stack of thin films. Thermal emission from such structures can be tailored to meet the requirements of different applications that require emissivity control, such as camouflage, selective 


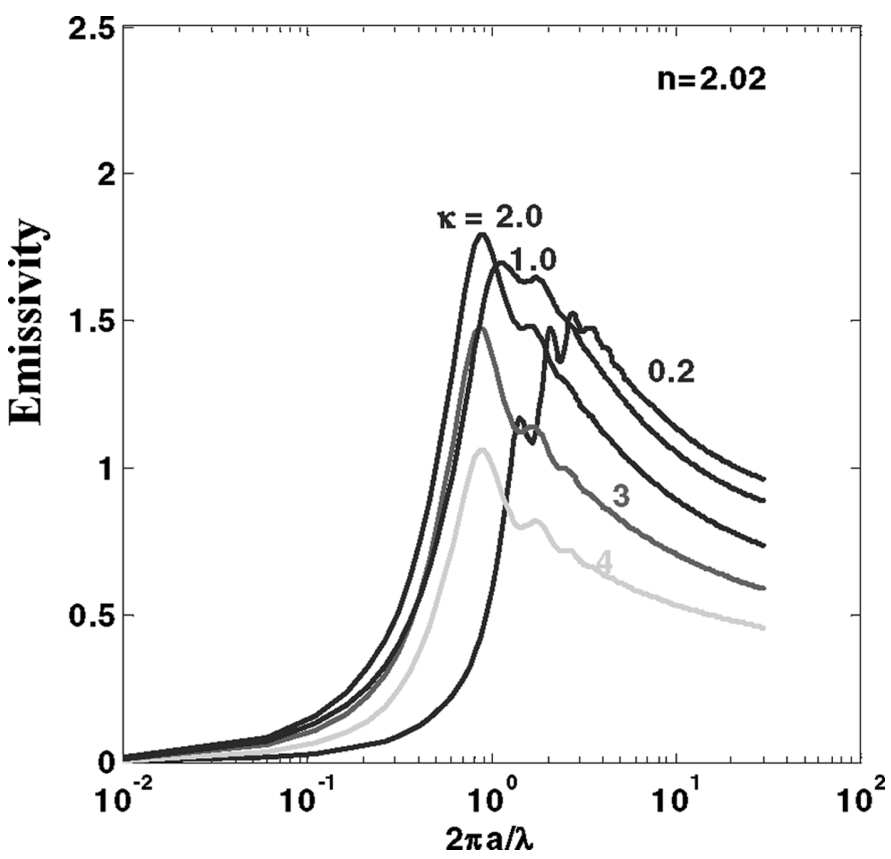

Figure 9. Emissivity of a spherical particles of radius a and refractive index $(n, k)$.

emitters for thermophotovoltaics, and windows. Despite some initial experimental results suggesting that emission from 3D photonic crystals can exceed that of blackbody, calculations show the contrary and it is now generally accepted that far-field emission from photonic crystals cannot exceed that of blackbody [59].

Since Planck stated that one condition for the validity of the blackbody radiation is that the object is in thermal equilibrium, it is interesting to ask whether there are examples that thermal emission can exceed that of blackbody radiation in nonequilibrium conditions. The answer is yes. Examples are the emission from a photodiode and radiative recombination emission from solar cells.

In engineering radiation problems, emissivity is treated as a surface property. However, thermal emission is a volumetric process, as the emission emerging from the surfaces really originates from inside the material. Direct calculation of the emission is usually based on the approach pioneered by Rytov [61, 62]. However, direct thermal emission calculation is difficult for complex structures $[58,59]$. There are some interesting problems where direct computation of thermal emission and radiation exchange between surfaces are desirable [63], and further development of the direct emission computational method is needed. It is also interesting to point out that the very concept of emissivity of nanostructures is debatable.

\section{Near-Field Radiation Heat Transfer}

Although it is difficult to exceed the vacuum blackbody radiation heat transfer limit in the far field, it is relatively easy to do so in the near field - when the separation 
between two objects exchanging photons is less than or equal to that of the photon wavelength. A well-established example is radiative heat transfer between two parallel plates separated by a distance of the order of the wavelength [64]. Both modeling and experiments have shown that thermal radiation can exceed that of the blackbody radiation in free space due to tunneling of evanescent waves. For two identical objects with a refractive index $\mathrm{n}$, the maximum thermal radiation exchange happens when the two surfaces are in contact. Thermal radiation heat transfer between the two objects is roughly now $\mathrm{n}^{2}$ times that of far-field heat transfer between the two surfaces, since blackbody radiation intensity inside the object is proportional to $\mathrm{n}^{2}$ for a weakly absorbing medium.

More recently, consideration of near-field radiation heat transfer has moved beyond two regular planar surfaces. A few topics are being pursued by different groups. One is the radiation heat transfer between surfaces that supports surface waves such as surface plasmons [65] and surface phonon-polaritons [66, 67]. Unlike evanescent waves that decay exponentially only on one side of the interface, surface waves decay exponentially on both sides of the interface. These surface waves are hybrid states of photons and electrons (surface plasmons) or photons and phonons (surface phonon-polaritons). Such surface waves can have a much larger density of states than that of free space. In addition, these waves can have quite long propagation lengths arising from the large propagation speed. The high density of states leads to a high heat flux between two closely spaced surfaces when the surface waves tunnel across the gap from one material into the other. This character is being explored for applications such as more efficient thermophotovoltaic energy conversion. The long propagation length means that the emission is spatially coherent [53]. When coupled to the far field by gratings, thermal emission from such surface waves are highly directional and nearly monochromatic. The long propagation length can also be used to channel heat along the film plane direction [68]. In addition to planar geometries, near-field radiation heat transfer has been extended to other geometries such as radiation heat transfer between a point and a planar surface and between two nanoparticles [69].

Surface waves warrant further discussion in the context of ongoing research in superlenses and negative index materials [70]. Taking the surface plasmon as an example, the dispersion relation (frequency and wave vector) of the surface plasmon can be expressed as

$$
\kappa_{S P}=\frac{\omega}{c} \sqrt{\frac{\varepsilon_{1} \varepsilon_{2}}{\varepsilon_{1}+\varepsilon_{2}}},
$$

where $\kappa_{S P}$ is the wave vector of the surface plasmon, $\omega$ is the angular frequency, $c$ is the speed of light, and $\varepsilon_{1}$ and $\varepsilon_{2}$ are the dielectric functions of the materials on either side of the interface. Surface waves exist when the denominator approaches zero, which occurs when the dielectric functions of the two materials at the interface are of opposite signs but equal in magnitude. This happens when the material vibrations are in resonance with the electromagnetic field. In this case, the dielectric function can be negative; i.e., the displacement field is in the opposite direction of the electric field. It is also possible that some materials, either by nature (which is rare) or artificial design, have negative magnetic permeability at that same frequency. In this case, it 
turns out that the refractive index must take a negative sign. Materials with such characteristics are called negative index materials, left-handed materials, or electromagnetic metamaterials [71]. Such materials have many interesting characteristics and their radiative properties are a subject of a few studies.

In addition, intentional termination of surfaces on photonic crystals can also lead to interesting surface waves. Some recent work in this area leads to interesting observations and further work can be fruitful $[72,73]$.

\section{Microscopic vs. Macroscopic Maxwell Equations}

All of the above discussions can start from the macroscopic Maxwell equations that rely on bulk materials properties such as dielectric functions to describe the wave propagation. When the structures become extremely small - for example, the case of monoatomic layer adsorbed on a surface in photoelectrochemical solar cells - the validity of applying macroscopic Maxwell's equations with bulk optical properties should be questioned. However, microscopic Maxwell's equations are still valid. Figure 10 shows the microscopic and macroscopic Maxwell's equations. The dielectric constant $\mathbf{D}$ is related to the electric field through

$$
\mathbf{D}=\varepsilon_{o} \mathbf{E}+\mathbf{P}=\overleftrightarrow{\varepsilon} \mathbf{E}
$$

where $\vec{\varepsilon}$ is a $3 \times 3$ tensor, although we usually treat it as a scalar $\varepsilon$ for bulk isotropic media, and $\mathbf{P}$ is the dipole displacement that is proportional to the local electric field $\mathbf{E}_{\text {loc }}$. This $\mathbf{E}_{\text {loc }}$ is different from the macroscopic electric field $\mathbf{E}(\mathbf{r})$ in that $\mathbf{E}_{\mathrm{loc}}$ is the field acting on the local dipole that also includes the induced electric field of other dipoles in the object. The relationship between the dielectric constant of a medium and the polarizability $\alpha_{j}$ of atom $j$ is given by the Clausius-Mossotti relation,

$$
\frac{\varepsilon-1}{\varepsilon+2}=\frac{1}{3 \varepsilon_{o}} \sum_{j} N_{j} \alpha_{j}
$$

\begin{tabular}{|c|c|c|}
\hline Microscopic & & Macroscopic \\
\hline $\begin{array}{l}\nabla \times \vec{e}=-\frac{\partial \vec{b}}{\partial t} \\
\nabla \times \vec{b}={ }_{0} \vec{j}+\frac{1}{c^{2}} \frac{\partial \vec{e}}{\partial t} \\
\nabla \cdot \vec{e}=/{ }_{0} \\
\nabla \cdot \vec{b}=0 \\
\vec{j}: \text { microscopic electric } \\
\quad \text { current density } \\
\text { microscopic } \\
\text { charge density }\end{array}$ & $\begin{array}{c}\langle\rangle \text { Spatial Averaging } \\
\vec{E}(\vec{r}, t)=\langle\vec{e}(\vec{r}, t)\rangle \\
\vec{B}(\vec{r}, t)=\langle\vec{b}(\vec{r}, t)\rangle\end{array}$ & $\begin{array}{l}\nabla \times \vec{E}=-\frac{\partial \vec{B}}{\partial t} \\
\nabla \times \vec{H}=\vec{J}+\frac{\partial \vec{D}}{\partial t} \\
\nabla \cdot \vec{D}= \\
\nabla \cdot \vec{B}=0 \\
\vec{D}=\vec{E} \\
\vec{B}=\vec{H}\end{array}$ \\
\hline
\end{tabular}

Figure 10. Macroscopic Maxwell equations are obtained from spatial averaging of microscopic Maxwell equations. 
where $N_{j}$ is the concentration of atom $j$. This relation is obtained based on the assumption of bulk materials and special lattice arrangements. It cannot be used for computing the dielectric constant of monolayers. Hu and Chen [74] considered the absorption of monolayers by constructing new dielectric constants and found appreciable differences between predictions of the macroscopic theory and results of microscopic Maxwell's equations.

\section{Coupling of Photons to Electrons and Phonons}

In considering photon interaction with materials, the absorption of photons is often treated as heat. There are, however, very interesting physical processes occurring from the photon absorption to the final heat generation. Equating photon absorption to heat generation sometimes leads to erroneous results. The optical refrigeration example discussed later is a good example. Absorption only means that photon energy is converted into another form of energy. In the case of interband transition in semiconductors, for example, the absorption of a photon generates one electron-hole pair. This electron-hole pair can radiatively recombine and emit light or nonradiatively recombine to generate heat. In the case that the electron-hole pair energy separation is much larger than the bandgap, it can also potentially generate more electron-hole pairs, which has significant implications for solar photovoltaic energy conversion.

Some of the phenomenon discussed above also can lead to development of new approaches for energy conversion. One example mentioned already is the use of surface plasmon wave tunneling for more efficient thermophotovoltaic cells [66]. Here, another example will be given. The tunneling of surface plasmons means that electrons in both sides of the surfaces are coupled. In nanoscale gaps, the large heat flux between electron systems on both sides of the interface suggests that it is possible to heat up electrons without heating up phonons, creating a nonequilibrium between electrons and phonons. It is suggested that this phenomenon can be explored to develop more efficient thermoelectric energy converters for power generation and refrigeration applications [75].

\section{LASER-BASED NANOPROCESSING}

\section{Introduction}

Fundamental understanding of microscale phenomena has been greatly facilitated in recent years, largely due to the development of high-resolution mechanical, electrical, optical, and thermal sensors. Consequently, new directions have been created for the development of new materials that can be engineered to exhibit unusual properties at submicron scales. Surface engineering is a critical subfield of nanotechnology because of the paramount importance of surface interaction phenomena at the micro/nano-machine level. Nanofabrication of complex three-dimensional patterns cannot be accomplished with conventional thermo-chemo-mechanical processes. While laser-assisted processes have been effective in component microfabrication with basic dimensions in the few-microns range, there is an increasing need to advance the science and technology of laser processing to the nanoscale. Breakthroughs in various nanotechnologies, such as information storage, optoelectronics, and biofluidics, can be achieved through basic research on nanoscale modification and 
characterization of surfaces designed to exhibit special topographical and compositional features at high densities.

Since their invention in the 1980s, scanning microscopy techniques such as scanning tunneling microscopy (STM), atomic force microscopy (AFM), scanning near-field optical microscopy, and further variants thereof have not only become indispensable tools for ultra-high-resolution imaging of surfaces and measurement of surface properties but have also offered hitherto unexplored possibilities to locally modify materials at levels comparable to those of large atoms, single molecules, and atomic clusters. These nanometric investigation tools have been extensively used in numerous high-resolution nanostructuring studies, to manipulate single atoms and also as effective all-inclusive nanofabrication tools. In view of rapid advances in nanotechnology, various applications and research fields are being envisioned, such as ultra-high-density data storage, mask repair, Fresnel optics for x-rays, nanoelectronics, nanomechanics, and nanobiotechnology, all of which depend on the fabrication of features only $5-10 \mathrm{~nm}$ in lateral dimensions. These feature sizes are more than an order of magnitude less than present industrial ultraviolet lithography standards. Due to the light diffraction that limits the size of the minimum resolvable feature to one-half of the wavelength of the radiation used, conventional optical lithography may not be applicable at the nanoscale. Although other techniques, such as electronbeam, ion-beam, and x-ray lithography, provide a means for high-resolution nanoprocessing, the excessive equipment cost, low processing speeds, and lack of materials suitable for fabricating various optical elements highlight the need for alternative and/or complementary process schemes.

Lasers are effective material processing tools offering distinct advantages, including choice of wavelength and pulse width to match the target material properties, one-step direct and locally confined structural modification [76]. In most cases, laser ablation is induced and/or accompanied by a thermal process due to sample heating. Accordingly, it is practically impossible to fabricate ablated features smaller than the size of the heat-affected zone (HAZ). Short-pulsed lasers are especially useful for precise processing of electronic materials because they can apply extremely highenergy flux while minimizing the HAZ. The optical penetration depth is dictated by the wavelength of the laser light and the complex refractive index of the sample material. Reduction of the minimum feature size is required not only to fabricate higher density devices but also to take advantage of scale-down effects. The minimum feature size achieved by widely used mask projection-based photolithography technology is also determined by the diffraction limit, again approaching $\lambda / 2$. One approach toward reduction of the minimum feature size is by utilizing shorter wavelengths, but this is clearly restricted as deep ultraviolet laser or x-ray radiation is needed. An alternative is to overcome the diffraction limit by nontraditional technology. In this context, the optical near field formed in the close proximity of tiny optical apertures has been employed. When light is irradiated onto an aperture whose diameter is smaller than the wavelength, the emerging radiation rapidly diverges due to diffraction. Before diverging, the light is physically confined in the immediate vicinity of the opening. This short range is defined as the optical near field and is roughly on the order of the aperture diameter [77]. Precise control of the gap distance between the aperture edge and the sample top surface is therefore required for optical near-field illumination. This has been realized by implementing scanning probe microscopy (SPM) technology such as atomic force microscopy (AFM) scheme. 
Confinement of optical energy to small dimensions can be achieved by coupling laser irradiation to near-field scanning optical microscopes (NSOMs) [78]. Scanning near-field optical microscopy (NSOM) was established as a powerful nanoscale characterization tool. Materials processing applications have been performed [79]. Organic film ablation tests using optical near-field schemes have been carried out [80]. In the context of applications, fiber probe ablation of thin metal films has been utilized for the fabrication or repair of metal photolithography masks [81]. Since laser sources are available with different photon energies and pulse lengths, matching with the absorption properties of the substrate can be dictated, in order to accomplish local chemical and structural modification with precision, accuracy, and without detrimental thermomechanical side effects.

The main technical challenge stems from the difficulty in transmitting pulses of sufficient energy for nanostructuring through nanoscale openings. However, nanoscale laser energy deposition has been shown to impart precise surface modification, even at levels sufficient for ablative material removal. The surface topography, texture, crystalline structure, and chemical composition could be modified with lateral feature definition in the nanometer range by coupling nanosecond and femtosecond laser beams to NSOM probes in both apertured and apertureless configurations.

\section{Apertureless NSOM Based Nanomachining}

Surface nanostructuring of various materials including gold, gold/palladium, poly(methyl methacrylate), and polycarbonate by illumination of a scanning probe microscope (SPM) tip with nanosecond laser pulses has been demonstrated in the literature [82]. Although laser-assisted STM-based nanoprocessing schemes have yielded resolutions on the order of $\sim 30 \mathrm{~nm}[83,84]$, restrictions on the electrical conductivity of both the material to be processed and the scanning probe tip as well as the ambient working conditions make these methods unsuitable for a wide range of electrically nonconductive and biological materials. Laser nanoprocessing schemes coupled with AFM operated either in contact or noncontact mode provide effective means for overcoming aforementioned drawbacks and enable the development of nanoscopic features of lateral dimensions less than $20 \mathrm{~nm}$. In spite of the increasing interest in this nanostructuring scheme, the actual processing mechanism still remains unexplained. In most cases, AFM or STM configurations were used with the tip in actual contact or in very close proximity (e.g., 3-5 $\AA$ ) with the sample surface, which complicated the identification of the basic modification mechanism.

Surface nanostructuring was accomplished with near-IR femtosecond pulses $[85,86]$ (Figure 11). Electric field intensity distributions were obtained with the finite difference time domain (FDTD) method [87]. A three-dimensional simulation was performed in which the four Maxwell's equations and two constitutive relationships were solved in conjunction with the current relationship. An enhancement of the field intensity can be seen at the Au film surface and the tip apex. The electric field distribution at the tip exhibits the lightning rod effect, which is more pronounced for longer wavelengths [88]. The lateral confinement of the field, with a FWHM of $\sim 10 \mathrm{~nm}$ clearly seen from the graphs, is believed to be the main factor for the high spatial resolution observed in the nanostructuring experiments.

The actual field strength enhancement may differ due to various influencing factors, such as approximations in the modeling of the actual tip shape, increase in the 

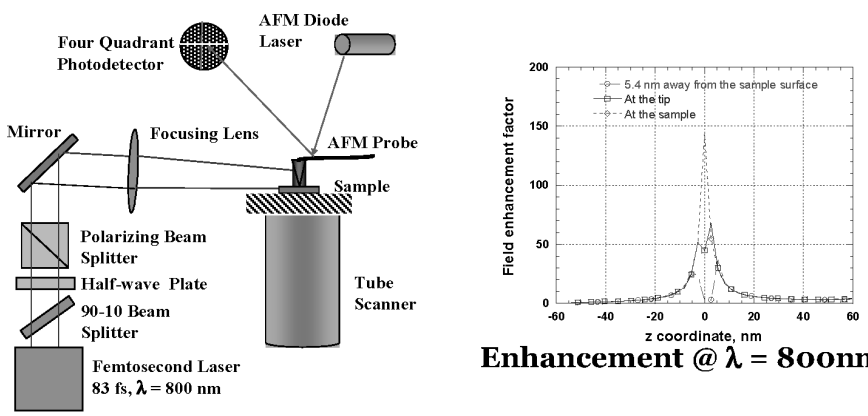

Enhancement @ $\lambda=$ soonm

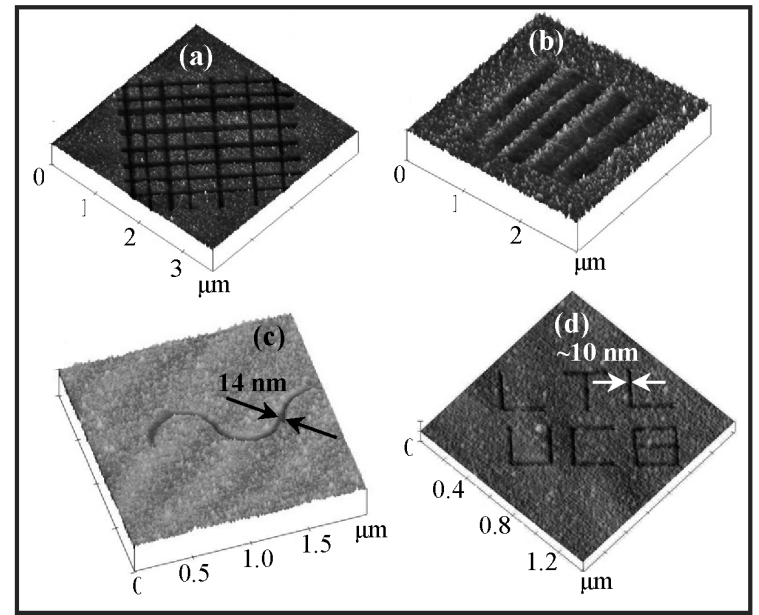

Figure 11. Schematic diagram of the apertureless near-field scanning optical microscope experimental setup for surface nanostructuring. The normalized electric field intensity distributions shown are for $\mathrm{Si}$ probe tip irradiated with P-polarization femtosecond laser of wavelength $\lambda=800 \mathrm{~nm}$. The tip apex/sample surface separation is $2.7 \mathrm{~nm}$. Surface nanostructuring of (a, c, d) $\sim 25$-nm-thick Au film and (b) 15-nmthick Au film.

effective tip-sample separation distance during processing due to the removal of material, surface roughness, native oxide films on the tip and sample surfaces, and in situ change of the tip apex radius as a result of using the same tip for both scanning and nanostructuring. While the nominal optical absorption depth $z_{s}=12 \mathrm{~nm}$, a modified optical absorption depth $z_{s}=1 \mathrm{~nm}$ was fitted to the computed near-field absorbed energy density profile in the target obtained from the FDTD near-field simulations.

Several investigators have attempted to explain the physical phenomena responsible for surface nanostructuring [89-91]. In general, material removal by ablation can be associated with photothermal, photomechanical, and photochemical effects. Ultrahigh-resolution fluorescence microscopy utilizing two-photon excitation and sharp metal tips and spectroscopy of single molecules have been demonstrated using similar configurations [92]. Direct observations of the enhanced field using photosensitive films have confirmed the nanoscopic spatial confinement of the electric field, hence excluding the possibility of surface modification due to the tip expansion [91]. The 
favorable sharp tip geometry (leading to the lightning rod effect discussed earlier) and the multiple scattering between the resonantly coupled probe tip and the sample structure thus effectively lead to the focusing of the optical power by collecting over the larger, nearly flat probe surface and reradiating over the smaller and sharper tipapex structure.

\section{Apertured NSOM-Based Nanomachining}

In the most common realization of apertured NSOMs, the optical fiber is coated by a cladding layer, typically aluminum, because of its high reflection at visible wavelengths. The transmission efficiency through the fiber probe is defined as the ratio of output energy to the input coupled energy and is roughly proportional to $(d / \lambda)^{n}$ ( $n$ is around 2-4, depending on the probe design) [93]. Because considerable input laser pulse energy is required to offset losses from apertures of decreasing size, the trade-off between minimum aperture size and minimum required light throughput should be considered in materials processing applications. The throughput of the NSOM chiefly depends on the geometry of the tapered region where the transmission efficiency drops as the fiber diameter becomes smaller than the laser wavelength.

Ablated craters by femtosecond laser pulses (wavelength and $\sim 100$ fs pulse width) are shown in Figure 11(a). The negative part of the machined feature was less than $200 \mathrm{~nm}$ in width and penetrated through the thickness of the metal film. Benefits of processing with shorter laser pulses are reduction of the thermal diffusion length, tighter concentration of the deposited energy and production of sharper features. When a solid sample is ablated using conventional lens focusing optics, the final shape of the ablated features is sensitive to the environment conditions. For example, when picosecond laser light is focused onto the solid sample, electrons are first excited and emitted almost instantaneously ( $\sim 100 \mathrm{fs}$ ) due to high mobility and small heat capacity.

If the sample is exposed to air, the emitted electrons collide with air molecules, triggering surface electron-initiated air plasma by ionization. Once the plasma is formed, it can further absorb the laser pulse energy for the remaining period of the pulse duration by inverse Bremsstrahlung, giving rise to a hot and dense state. The energy stored in the electron subsystem in the target solid is transferred to the lattice at the timescale of $\sim 0.1-10$ ps due to the much higher heat capacity of the lattice compared to electrons. Therefore, the material ejection process commences after an elapsed time of about 10-100 ps. Upon interacting with the air plasma, the ejecta can bounce back and compress the melt. This can explain why much cleaner features can be fabricated in vacuum or nonreactive gas environments such as helium. In nanosecond ablation, the incident beam interacts with the ejecta over a substantial fraction of the pulse duration. Consequently, the ablation threshold is not very sharp because of the more complex plasma shielding effect compared to femtosecond laser ablation. In the case of ablation through NSOM fiber probes, the laser passes through a narrow, approximately nanometer-scale gap before reaching the sample. The laser interaction with the plasma is therefore expected to be weaker compared to lens focusing scheme. This argument is supported by the clear dependence of the feature size on the laser pulse energy applied for both nanosecond and femtosecond laser pulses as shown above (nanosecond results given in Hwang et al. [94]). However, the topography of features ablated in ambient air exhibit qualitative difference compared with features ablated in vacuum [Figure 12(b)]. 

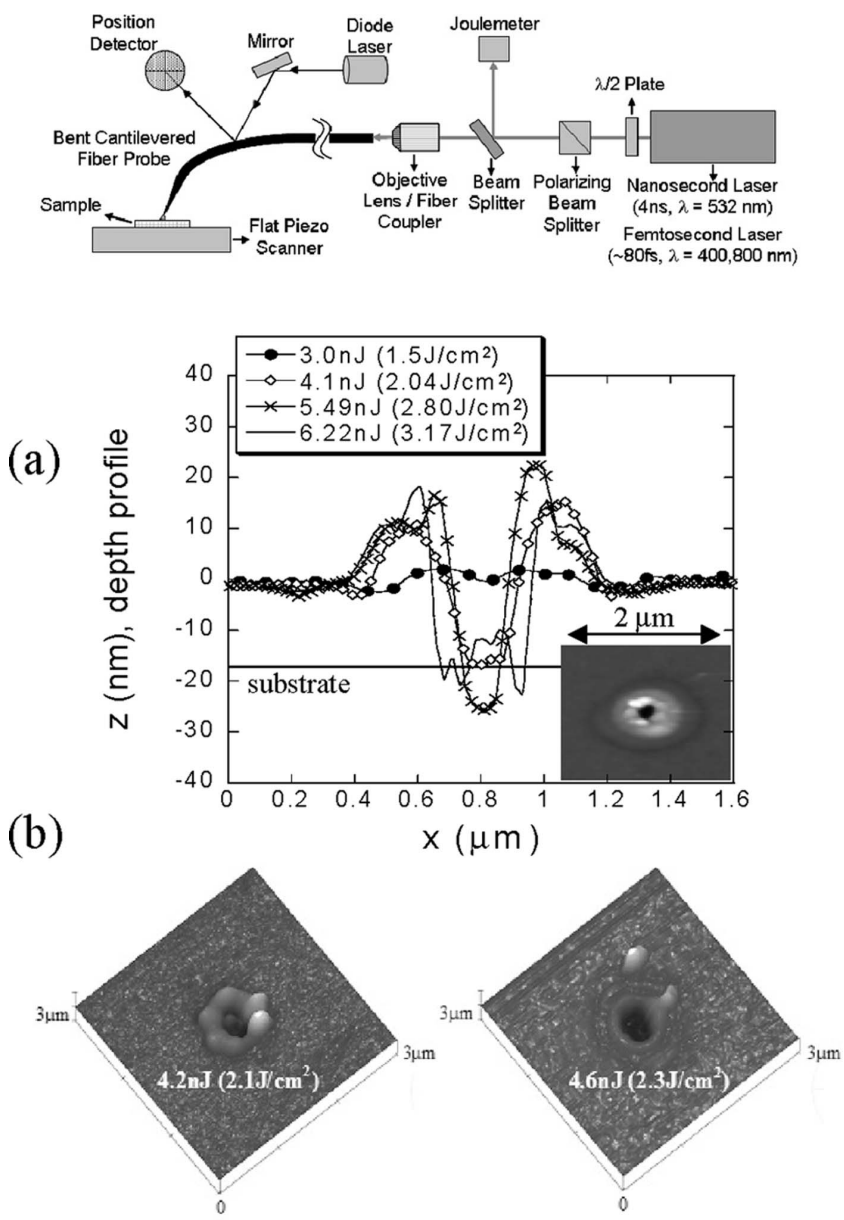

Figure 12. The top figure gives a schematic of the experimental setup for processing utilizing a cantilevered NSOM system. (a) Ablation crater profiles by femtosecond laser (500 nm probe, $400 \mathrm{~nm}$ wavelength) as laser energy varies; (b) comparison of ablated craters in ambient air (left) and moderate vacuum (right) [94].

\section{Nanoscale Melting and Crystallization}

In erasable phase change optical data storage applications, a "bit" of information is written on a crystallized region of the disk using a sufficiently strong laser pulse to induce local melting and rapid cooling leading to formation of amorphous phase [95]. This bit can be read by using a low-energy laser pulse and noting the optical response difference compared to the surrounding crystalline region. Furthermore, to erase this bit one can use intermediate laser pulse energy to raise the temperature of the film above the crystallization temperature but below the melting temperature, thus converting the amorphous region back into its corresponding crystalline phase to erase optical contrast. Understanding the nanoscale amorphization and recrystallization phenomena using optical near-field techniques is essential for ultra-highdensity data storage applications. 

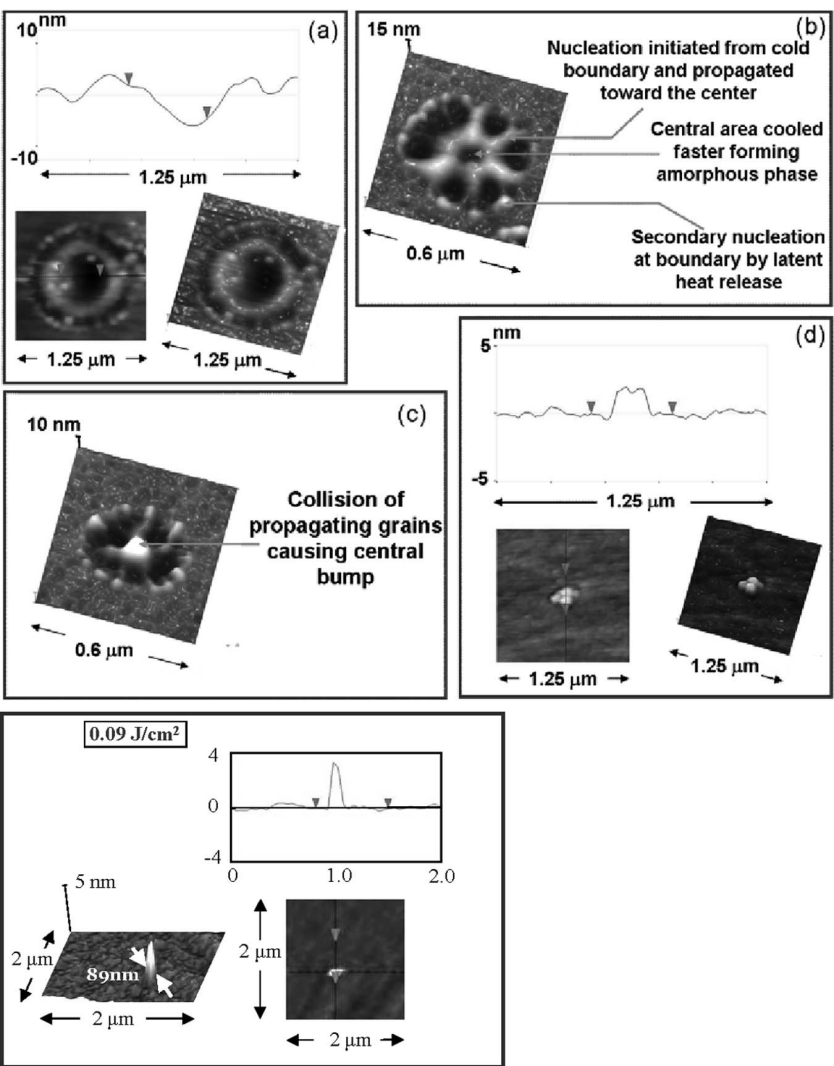

Figure 13. The upper panels depict limiting cases of nanocrystallization study of 20-nm-thick a-Si films based on apertured NSOM for laser fluence equal to (a) $5.9 \mathrm{~J} / \mathrm{cm}^{2}$, (b) $3.3 \mathrm{~J} / \mathrm{cm}^{2}$, (c) $3.0 \mathrm{~J} / \mathrm{cm}^{2}$, and (d) 2.0 $\mathrm{J} / \mathrm{cm}^{2}$. The figure below shows a $90-\mathrm{nm}$ feature nucleated by the apertureless NSOM scheme. The fluence in this case corresponds to the beam incident to the Si tip.

Both the apertureless and apertured NSOM schemes can be employed to explore the nanoscale melting and crystallization phenomena. Representative nanocrystallization results for the 20-nm-thick a-Si films deposited on quartz with the Nd:YAG pulsed nanosecond laser obtained with the aforementioned two schemes are shown in Figure 13 [96]. Ablation is observed at very high energy density. With gradual decrease in energy, but at still high enough fluence values, the central region melts completely, whereas the adjacent surrounding area is partially melted. Therefore, nucleation first occurs in the outer region, as evidenced by the formation of small polycrystals. Grains launched from these seeds grow laterally toward the central and completely molten region until their growth is arrested by impingement with lateral grains growing from the opposite direction or by spontaneous nucleation triggered in the severely supercooled molten silicon pool [97]. Considering the solid/liquid interfacial kinetics [98], and experimental time-resolved imaging evidence [99], the lateral crystal growth speed is around $10 \mathrm{~m} / \mathrm{s}$. Consequently, the elapsed time for the lateral crystal growth would be approximately 20-30 ns. Distinct modification regimes produced as a result of the rapid melting and crystallization of the a-Si films can be clearly identified. Due to the higher peak temperature at the center of the melt pool and because nucleation usually occurs at 
the solid/liquid phase boundary, polycrystalline grains growing toward the center of the supercooled melt were observed at high fluences. For a lower fluence, a bump was produced at the crystallized spot center due to collision of the silicon crystals. Another regime characterized by the formation of a ring consisting of individual grains was encountered when the fluence decreased. The lateral size of these nanostructures was typically in the range of 70-80 $\mathrm{nm}$. Further decrease of the input laser fluence resulted in the formation of a single nanostructure with a lateral dimension of $\sim 90 \mathrm{~nm}$. The size of these nanostructures could be further confined by using even lower fluences and sharper tips, although limitations due to thermal diffusion would still exist.

\section{NSOM-Based Chemical Processing}

Both pyrolytic and photolytic LCVD have the ability to define submicron features, not only for achieving high-quality deposition of a wide range of materials on a variety of substrates but also very significantly for fabricating 3-D nanostructures [100]. Recent experiments have yielded successful results for the pyrolytic NSOMLCVD deposition of sub-100-nm Si dots on crystalline Si by the decomposition of $\mathrm{SiH}_{4}$ [101]. Fabrication of three-dimensional objects of nanometric dimensions and arbitrary shape may be accomplished by extending the deposition and crystal growth through precise motion of the substrate.

\section{Summary}

Surface nanostructuring at high spatial resolution can be performed with great accuracy and reproducibility by NSOM schemes. The obtained nanostructure features with minimum lateral dimensions of $\sim 10 \mathrm{~nm}$ and various complex nanopatterns illustrate the flexibility of the experimental techniques. Possible applications of this nanostructuring process are envisioned in high-resolution nanolithography, controlled nanodeposition, ultra-high-density data storage, mask repair, nanoelectronics, nanophotonics, various nanobiotechnology applications, as well as nanoscale chemical analysis and imaging.

From the fundamental point of view, it is important to understand in detail the transport phenomena involved in nanoscale laser processing. As the lateral extent of the imparted radiation shrinks to the nanometric regime, the near-field absorption distribution exhibits significant departures from the classical Beer's law exponential decay. Furthermore, the surface reflectivity prediction based on the refractive index is not relevant as the near-field optical electromagnetic distribution has to be derived from detailed computations. Confinement of the radiative energy distribution leads to increased lateral diffusion that in turn requires higher coupled pulse energy and consequently more efficient near-field optics. As the material melts, the extremely strong temperature gradients developed will strongly perturb the surface. At higher intensities, the ablative regime kicks in. Even though the probe/surface gap is extremely narrow, in the nanometer or tens of nanometers range, qualitative difference in ablation craters under vacuum environment is observed. Very little is known about the plasma dynamics and shock wave generation under these extremely localized energy transfer and probe proximity constraints. Detailed, time-resolved in situ probing of the thermophysical conditions of the irradiated surface, the emitted radiation, and the ejected plasma is crucial while analytical and numerical modeling can be utilized to interpret the experimental results. 


\section{AB INITIO CALCULATION OF PHOTON-ELECTRON-PHONON COUPLINGS IN LASER COOLING OF ION-DOPED SOLIDS}

As an example of energy exchange at the atomic level, laser cooling of solids is discussed. The photon (propagating electromagnetic field) coupling with the outer shell electron (of ion dopant) and then the coupling of this excited electron with the lattice vibration (phonon) are discussed. These two coupling factors are given in terms of the electron wave functions and the perturbed Hamiltonian, quantities that need to be determined from ab initio calculations. The steps for these calculations are outlined.

\section{Introduction}

Using lasers, gases have been cooled to extremely low temperature of the order of $\mathrm{nK}$ [102-105]. Laser cooling of solids has also attracted interests recently (with application in optical cryo-cooled sensors). Pringsheim introduced the concept of laser cooling (optical refrigeration) of solids, recognizing that the thermal vibrational energy (phonon) might be removed by the anti-Stokes fluorescence; i.e., the photons emitted by an optical material have a mean energy higher than that of the absorbed photons [106]. This idea was initially believed to contradict the second law of thermodynamics. Predictions suggested that the cycle of excitation and fluorescence was reversible, and hence the optical cooling would be equivalent to the complete transformation of heat to work [107, 108]. This issue was cleared by Landau by assigning entropy to radiation [109]. It was shown that the entropy of a radiation field is proportional to its frequency bandwidth and also to the solid angle through which it propagates. Since the incident laser light has a very small bandwidth and propagates in a well-defined direction, it has almost zero entropy. On the other hand, the fluorescence is relatively broadband and is propagating in all directions and therefore it has comparatively larger entropy $[110,111]$. In this way, the second law of thermodynamics is satisfied.

Many attempts have been made to realize radiative refrigeration experimentally, and the associated theoretical interpretations have been proposed. The earliest experiment was performed by Kushida and Geusic on Nd:Yag [112]. Reduced heating other than net cooling was observed, which was conjectured to be a result of the impurities in the crystal and the multi-phonon decay across the optical transition. Later Djeu and Whitney laser-cooled low-pressure $\mathrm{CO}_{2}$ solid by $1 \mathrm{~K}$ from $600 \mathrm{~K}$ by using a $\mathrm{CO}_{2}$ laser for pumping [113]. In 1995, Epstein et al. [114] reported the first successful experiment of laser cooling in solids. Since then, various $\mathrm{Yb}$ - or Tm-doped glasses and crystals have been cooled [115-124]. In particular, bulk solids have been cooled from room temperature to $208 \mathrm{~K}$ (creating a temperature difference $\Delta T=92 \mathrm{~K}$ ) [124]. Continuous progress has been made [122, 125, 126] toward achieving cryogenic temperatures. For semiconductors, theoretical predictions have shown their potential to be cooled to as low as $50 \mathrm{~K}$ starting from the room temperature [127], but experimental success is yet to be achieved due to some serious challenges (including potential for total reflection and reabsorption of the fluorescent emission).

These advances, as well as those in laser cooling of gases (achievement of BoseEinstein condensate) [102-105], has further stimulated interest in optical cooling of solids. 


\section{Principal Mechanisms in Laser Cooling of Solids}

Laser cooling can be viewed as the inverse cycle of lasers, and laser materials are in principle also good candidates for laser cooling. Common laser materials in the solid state include ion-doped solids and semiconductors, which are currently being studied for laser cooling.

Figures 14(a) to (e) show the energy diagram, as well as the steps identifying the mechanisms, in laser cooling of an ion-doped dielectric solid. There host crystal lattice is idealized as transparent to the pumping laser. Some of its atoms are replaced by optically active, doped ions (e.g., $\mathrm{Yb}^{3+}$ ). The ion is represented by an effective transition dipole moment, which is the matrix element of the dipole operator $\boldsymbol{\mu}_{e}$ [128]; i.e.,

$$
\boldsymbol{\mu}_{e}=\int \psi_{f}^{*} e_{e} \boldsymbol{r} \psi_{i} d^{3} \boldsymbol{r}
$$

where $e_{e}$ is the electron charge, $\boldsymbol{r}$ is the position vector, and $\psi_{i}$ and $\psi_{f}$ are the initial and final state wave functions of the two-level system. The matrix element couples the two states, which have different parity, creating or absorbing a photon. The electromagnetic field, which has a polarization vector $\boldsymbol{e}_{\alpha}$, may interact with the ion if the coupling factor $\mu_{\mathrm{e}} \cdot \boldsymbol{e}_{\alpha}$ is nonzero (i.e., they are not orthogonal). The principal mechanisms of the photon-electron-phonon interactions resulting in the cooling effect in the solid, are shown in Figures 14(a)-(e). In step (a), the electron in the ground state is coupled to a phonon and forms a combined state. In (b), when the medium is irradiated by laser light with a frequency $\omega_{p h, i}$ that is below the resonance frequency $\omega_{e, g}$ for the energy gap $\left(10,250 \mathrm{~cm}^{-1}\right)$ for $\mathrm{Yb}^{3+}$ ion in $\mathrm{Y}_{2} \mathrm{O}_{3}$, the electron has some probability to be excited by absorbing a photon from the pumping field and the coupled phonon, such that $\omega_{p h, i}+\omega_{p}=\omega_{e, g}$. According to the Born-Oppenheimer approximation, this electronic transition is instantaneous and the ion core does not move. In (c), after the electron is in the excited state, the charge distribution around the ion has been alternated, and the lattice will relax to new equilibrium positions. In (d), the electron then undergoes a radiative decay and returns to the ground state, emitting a photon with frequency $\omega_{p h, e}$. It is followed in (e) by another lattice relaxation process with restores the lattice to its original positions.

\section{Population Rate Equation and Cooling Power}

In Figure 15, a macroscopic energy diagram is show for a solid that is cooled by laser, where various energy flows are shown. The energy entering the control volume is the laser irradiation power $Q_{p h, i}$, and those leaving the control volume are the unabsorbed power $(1-\alpha) Q_{p h, i}$ and the fluorescence emission power $Q_{p h, e}$. The net cooling power $\dot{S}_{p h-e-p}$ is given by $\alpha Q_{p h, i}-Q_{p h, e}$ and is balanced by the external thermal load. To minimize the external thermal load to achieve the most cooling effect, in most of the existing experiments the solid was supported by very thin wires and was placed in vacuum, to eliminate the conduction and convection. The only external load is then the thermal radiation from surroundings, which is at temperature $T_{\infty}$, as shown in Figure 15.

In order to relate the absorbed and emitted power, the population dynamics of the ground and excited electronic states needs to be established. Although the rate equation is not suitable for describing atoms with any correlations between them (such 


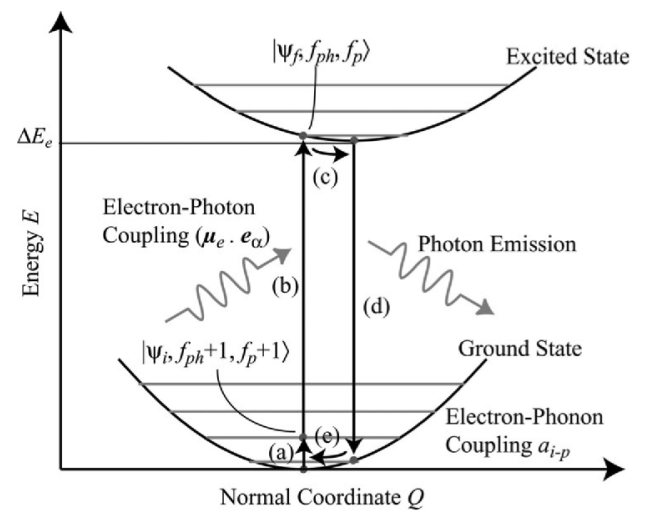

Steps in laser cooling process
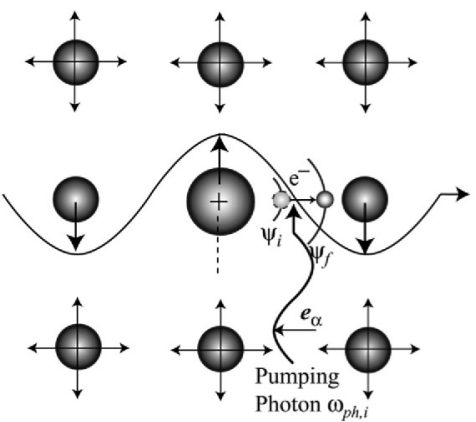

(b) Phonon Assisted Absorption (under Born-Oppenheimer Approximation)

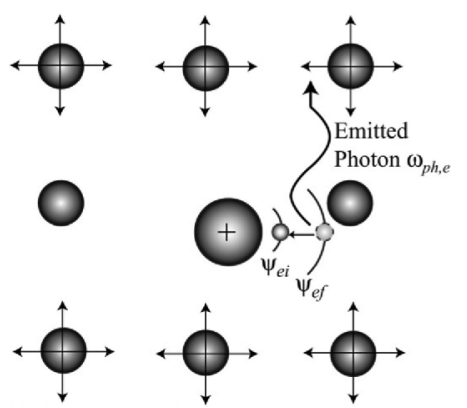

(d) Emission (under Born-Oppenheimer Approximation), Ending in Ground State (Boltzmann Statistics)

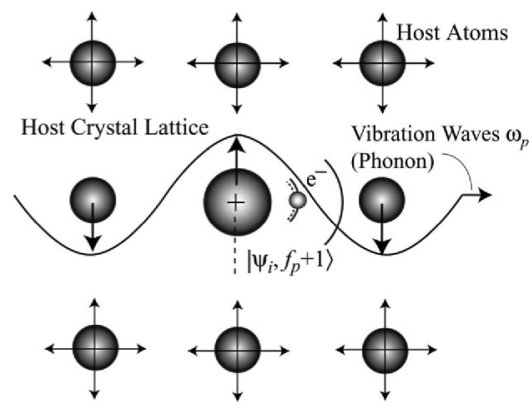

(a) Electron-phonon Coupling

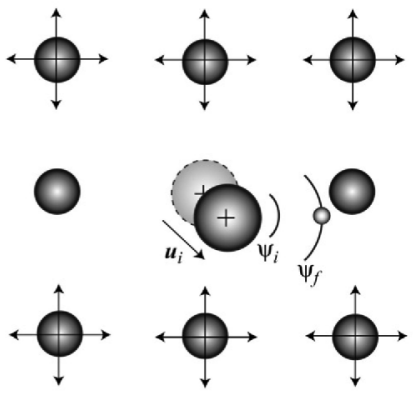

(c) Lattice Relaxation and Displacement (under Energy Minimization)

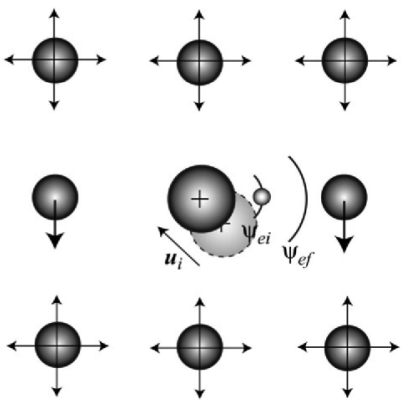

(e) Lattice Relaxation and Restoring (under Energy Minimization)

Figure 14. (a) The energy diagram, (b)-(e) five steps in the photon-electron-phonon couplings in the energy transfer cycle, for the laser cooling of ion-doped solids.

as in cooperative interactions or other interatomic couplings), it is correct to describe the population dynamics of statistically independent atoms. In the nanoparticles studied, cooperative effects are negligible [129]. Hence, the rate equation for the two-level system shown in Figure 14(a) is 


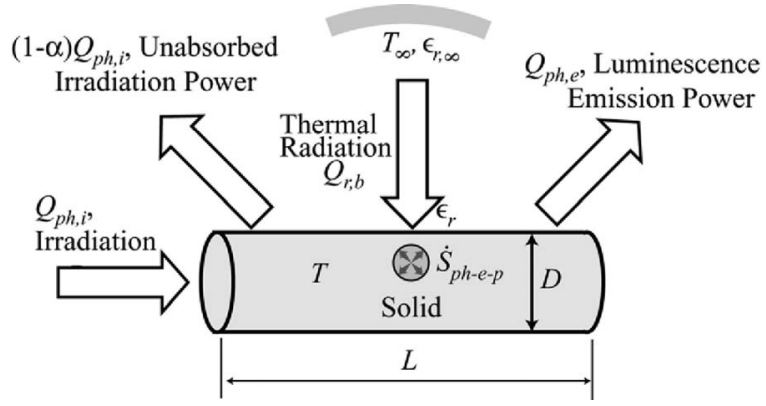

Figure 15. Overall energy conservation of a laser-cooled cylindrical solid under vacuum. Also shown are the various heat transfer mechanisms and the energy conversion.

$$
\frac{d n_{b}}{d t}=-\dot{\gamma}_{e, d} n_{b}+\dot{\gamma}_{e, a} n_{a}
$$

where $n_{b}$ and $n_{a}$ are the populations at the excited and ground states. Here, $\dot{\gamma}_{e d}$ is the total decay rate, contributed by both the radiative decay (includes the spontaneous and stimulated emissions) and nonradiative decay processes. The rate $\dot{\gamma}_{e, a}$ is the absorption (or excitation) rate.

Under steady-state, the total absorbed power is then given by

$$
Q_{p h, a}=\hbar \omega_{p h, i} \int_{V} \dot{\gamma}_{e, a} n_{a} d V
$$

Similarly, a radiative decay transition leads to the emission of a photon with a mean energy $\hbar \omega_{p h, e}$. The total emitted power is thus given by

$$
Q_{p h, e}=\hbar \omega_{p h, e} \int_{V} \dot{\gamma}_{e, r} n_{b} d V=\frac{\omega_{p h, e}}{\omega_{p h, i}} \eta_{e-p h} Q_{p h, a}
$$

where $\eta_{e-p h}$ is the quantum efficiency defined as $\eta_{e-p h}=\dot{\gamma}_{e, r} / \dot{\gamma}_{e, d}$.

The net cooling power $\dot{S}_{p h-e-p}$ is then determined as the difference between the absorbed and emitted radiation; i.e.,

$$
\dot{S}_{p h-e-p}=\left(1-\frac{\omega_{p h, e}}{\omega_{p h, i}} \eta_{e-p h}\right) Q_{p h, a}=\left(1-\frac{\omega_{p h, e}}{\omega_{p h, i}} \eta_{e-p h}\right) \hbar \omega_{p h, i} \int_{V} \dot{\gamma}_{e, a} n_{a} d V
$$

Thus, to enhance the cooling power using the same incident energy, increases in the dopant concentration $n_{d}$ and the excitation rate $\dot{\gamma}_{e, a}$ are necessary.

\section{Excitation Rate $\dot{\gamma}_{e, a}$ : A Fermi Golden Rule Calculation}

As the pumping wavelength is tuned to the red side of the resonance, the probability of a purely electronic transition between electronic sublevels, a 
first-order process, becomes smaller. On the other hand, the phonon-assisted transition, a second-order process, starts to contribute significantly to absorption. As a result, the absorption turns out to be a combination of the first- and second-order transitions. Since a much longer pumping wavelength than the resonance is used in laser cooling, the total transition is believed to be dominated by the second-order process. Therefore, only the second-order process will be analyzed here for the purpose of understanding the role of phonons in laser cooling.

In the second-order process, the ion in its ground state absorbs a photon from the irradiation and simultaneously a phonon from the lattice and goes up to the excited state. The probability per unit time of such a process can be evaluated using the perturbation theory. The Hamiltonian for the physical system considered is given by [119]:

$$
\mathrm{H}=\mathrm{H}_{i o n}+\mathrm{H}_{p}+\mathrm{H}_{p h}+\mathrm{V}_{\text {ion-ph }}+\mathrm{V}_{\text {ion-p }}
$$

where these Hamiltonians and potentials are given in [130].

The transition rate $\dot{\gamma}_{e, a}$ is given by the Fermi golden rule [119]:

$$
\dot{\gamma}_{e, a}=\sum_{f} \dot{\gamma}_{e, i-f}=\frac{2 \pi}{\hbar} \sum_{f}\left|M_{f i}\right|^{2} \delta\left(E_{f}-E_{i}\right)
$$

where $E_{i}$ and $E_{f}$ are the initial and final energies of the system. The $M_{f i}$ matrix can be expanded as the following series [119]:

$$
\begin{aligned}
M_{f i}= & \left\langle f\left|\varphi_{\text {int }}\right| i\right\rangle+\sum_{m} \frac{\left\langle f\left|\mathrm{~V}_{\text {int }}\right| m\right\rangle\left\langle m\left|\mathrm{~V}_{\text {int }}\right| i\right\rangle}{E_{e, i}^{T}-E_{e, m}^{T}} \\
& +\sum_{m, n} \frac{\left\langle f\left|\mathrm{~V}_{\text {int }}\right| m\right\rangle\left\langle m\left|\mathrm{~V}_{\text {int }}\right| n\right\rangle\left\langle n\left|\mathrm{~V}_{\text {int }}\right| i\right\rangle}{\left(E_{e, i}^{T}-E_{e, m}^{T}\right)\left(E_{e, i}^{T}-E_{e, n}^{T}\right)}+\cdots
\end{aligned}
$$

with $\mathrm{V}_{\text {int }}=\mathrm{V}_{\text {ion-ph }}+\mathrm{V}_{\text {ion-p }}$. The summations on $m$ and $n$ include all the intermediate phonon and photon states.

We plan to calculate the transition probability $\dot{\gamma}_{e, a}$ between the initial $|i\rangle=\mid \psi_{i}$, $\left.f_{p h}+1, f_{p}+1\right\rangle$ and final $|f\rangle=\left|\psi_{f}, f_{p h}, f_{p}\right\rangle$ states of the system, where the first ket element, $\psi_{i}$, refers to the ion state, the second one, $f_{p h}$, to the photon number in the interacting volume $V_{s}$, and the third one, $f_{p}$, to the phonon distribution function. This type of processes only appears in the second-order perturbation expansion of the $M_{f i}$ matrix, which is $[131,132]$

$$
\begin{aligned}
M_{f i, 2 n d}= & \sum_{m} \frac{\left\langle f\left|\mathrm{~V}_{\text {int }}\right| m\right\rangle\left\langle m\left|\mathrm{~V}_{\mathrm{int}}\right| i\right\rangle}{E_{e, i}^{T}-E_{e, m}^{T}} \\
= & \sum_{m}\left[\frac{\left\langle\psi_{f}, f_{p h}, f_{p}\left|\mathrm{~V}_{\text {ion-ph }}\right| \psi_{m}, f_{p h}+1, f_{p}\right\rangle\left\langle\psi_{m}, f_{p h}+1, f_{p}\left|\mathrm{~V}_{\text {ion-p }}\right| \psi_{i}, f_{p h}+1, f_{p}+1\right\rangle}{E_{i}-\left(E_{m}-\hbar \omega_{p h}\right)}\right. \\
& \left.+\frac{\left\langle\psi_{f}, f_{p h}, f_{p}\left|\mathrm{~V}_{\text {ion-p }}\right| \psi_{m}, f_{p h}, f_{p}+1\right\rangle\left\langle\psi_{m}, f_{p h}, f_{p}+1\left|\mathrm{~V}_{\text {ion-ph }}\right| \psi_{i}, f_{p h}+1, f_{p}+1\right\rangle}{E_{i}-\left(E_{m}-\hbar \omega_{p h}\right)}\right]
\end{aligned}
$$


The sum runs over all possible intermediate states of the system. This yields:

$$
\dot{\gamma}_{e, a}=\frac{2 \pi}{\hbar} \frac{\mathbf{e}_{\alpha} \cdot \mu_{e}}{2 \varepsilon_{\mathrm{o}}} \frac{a_{i-p}^{2}}{2 \rho u_{p}^{2}} \frac{D_{p}\left(E_{p}\right) f_{p}^{\circ}\left(E_{p}\right)}{E_{p}} e_{p h, i}
$$

where $\rho$ is the density, $D_{p}\left(E_{p}\right)$ is the phonon density of states, and $e_{p h, i}$ is the pumping field energy.

\section{Ab Initio Calculations of Coupling Factors}

Ab Initio Calculations In the electron-photon coupling Hamiltonian $\phi_{\text {ion-ph }}$ and electron-phonon coupling Hamiltonian $\phi_{\text {ion-p }}$, the electron-photon coupling factors $a_{e-p h}$ and the electron-phonon coupling factor $a_{i-p}$ is unknown. These need to be determined before the Fermi Golden Rule calculation. Since they are well related to electronic wavefunctions, ab initio methods should be a good choice.

In ab initio calculations, the Schrödinger equation is solved for the electron wave functions; i.e.,

$$
\mathrm{H} \psi\left(\mathbf{r}_{1}, \mathbf{r}_{2}, \cdots \mathbf{r}_{N}\right)=\mathrm{E} \psi\left(\mathbf{r}_{1}, \mathbf{r}_{2}, \cdots \mathbf{r}_{N}\right)
$$

where $\mathrm{H}$ is the total Hamiltonian and $\boldsymbol{r}_{i}(i=1-N)$ is the coordinate of the $i$ th electron. Since the unit cell of a solid often has a few or even tens of atoms, each atom with many electrons, it is not practical to solve this many-body problem precisely. Hence, a density functional theory has been developed as an approximation to the full Schrödinger problem. The basic idea is to decompose the many-body problem into many one-electron problems. The interactions between these electrons need to be addressed properly. For this purpose, a charge density is defined as

$$
\rho_{e}(\mathbf{r})=\sum_{i=1}^{N} \phi_{i}^{*}(\mathbf{r}) \phi_{i}(\mathbf{r})
$$

where $\phi_{i}$ is the stationary wave function of the $i$ th electron. The interaction between one electron and other electrons is then converted to the problem of one electron in a charge field. The Schrödinger equation for each electron becomes

$$
\mathrm{H} \phi_{i}(\mathbf{r})=\mathrm{E}_{i} \phi_{i}(\mathbf{r})
$$

where $E_{i}$ is the energy of the $i$ th electron, and the Hamiltonian $\mathrm{H}$ is approximated as

$$
\mathrm{H}=-\nabla^{2}+\mathrm{V}_{\text {ion }}(\mathbf{r})+\int \frac{\rho_{e}\left(\mathbf{r}^{\prime}\right)}{\mathbf{r}-\mathbf{r}^{\prime}} d \mathbf{r}^{\prime}+\mathrm{V}_{x c}\left[\rho_{e}(\mathbf{r})\right]
$$

where $\mathrm{V}_{\text {ion }}(\mathbf{r})$ is the potential of the ion on its valence electron, and many such pseudopotentials have been determined for different elements. The third term on the right-hand side is the classical electrostatic interaction, and the last term is the quantum effects correction on the previous term. To solve the problem, a trial charge 
density $\rho_{e}(\mathbf{r})$ is used in Eq. (42), then Eq. (41) can be solved for the wave function for each electron. These results are used in Eq. (40) to find a new $\rho_{e}(\mathbf{r})$. After a few iterations the converged solution is obtained.

Calculation of Photon-Electron Coupling Factor $\boldsymbol{a}_{\boldsymbol{p h}-\boldsymbol{e}}$ The photon-electron coupling factor $a_{e-p h}$ is given by

$$
a_{e-p h}=-\boldsymbol{e}_{\alpha} \cdot \mu_{e}
$$

where $\boldsymbol{e}_{\alpha}$ is the polarization vector, and $\boldsymbol{\mu}_{e}$ is the transition dipole moment that is defined in Eq. (29). To calculate $\mu_{e}$, the initial and final state wave functions need to be determined. These wave functions for a complex system as $\mathrm{Yb}^{3+}: \mathrm{Y}_{2} \mathrm{O}_{3}$ can be calculated using the $\mathrm{ab}$ initio approach and the transition dipole moment is readily determined.

Equation (29) also involves selection rules on the symmetry of the wave functions. The transition dipole moment is nonzero only when the two state wave functions have different symmetries.

Calculation of Electron-Phonon Coupling Factor $\mathbf{a}_{e-p}$ The ions in a crystal are in thermal motion and the thermal vibrations of these ions (phonons) modulate the local crystal field at the site of the optically active ion. This modulation may cause transitions to occur between electronic energy levels accompanied by the absorption or emission of phonons. When considering transitions, the thermal vibrations of the atoms need to be treated as quantized harmonic oscillators, which is similar to the treatment of the radiation field.

Considering the vibrations of a system of $N$ atoms on the crystal lattice, atom $i$ is displaced by an amount $\boldsymbol{u}_{i}$. Under harmonic approximation, the kinetic and potential energies of the vibrating atoms vibrating about their equilibrium positions is written as [132]:

$$
\begin{gathered}
\mathrm{T}=\frac{1}{2} \sum_{i=1}^{N} \sum_{\alpha=1}^{3} m_{i} \dot{u}_{i \alpha}^{2} \\
\mathrm{~V}=\frac{1}{2} \sum_{i=1}^{N} \sum_{j=1}^{N} \sum_{\alpha=1}^{3} \sum_{\beta=1}^{3} A_{i \alpha, j \beta} u_{i \alpha} u_{j \beta}
\end{gathered}
$$

where the $i$ and $j$ sums are over the atoms in the system, and the $\alpha$ and $\beta$ sums are over the $x, y$, and $z$ directions. Here $A_{i \alpha, j \beta}$ is the force constant. The Hamiltonian is given by

$$
\mathrm{H}=\mathrm{T}+\mathrm{V}
$$

Similar to the quantization of the radiation field, this vibrational field is quantized if the normal coordinates are introduced as

$$
Q_{q}^{\lambda}=\sum_{i, \alpha}\left(\frac{m_{i}}{N}\right)^{1 / 2} u_{i, \alpha} \varepsilon_{q \alpha}^{\lambda *} e^{-i \boldsymbol{q} \cdot \boldsymbol{r}_{i}}
$$


where $q$ ranges from 1 to $N$ and $\lambda$ ranges over the different acoustic and optical branches. The set $\varepsilon_{q \alpha}^{\lambda}$ are the eigenvectors corresponding to the normal modes. In terms of the creation and annihilation operators, the normal coordinate is written as:

$$
Q_{q}^{\lambda}=\left(\frac{\hbar}{2 \omega_{q \lambda}}\right)^{1 / 2}\left(b_{q}^{\lambda}+b_{-q}^{\lambda+}\right)
$$

and the Hamiltonian is

$$
\mathrm{H}=\sum_{q, \lambda}^{3 N} \hbar \omega_{q, \lambda}\left(b_{q}^{\lambda \dagger} b_{q}^{\lambda}+\frac{1}{2}\right)
$$

The electron-phonon interaction is manifested through the change in the crystal field due to the changes in the relative positions of the active ions and their surrounding ligands.

For acoustic phonon modes, the electron-phonon interaction is via the local strain defined by the tensor

$$
\varepsilon_{\alpha \beta}=\frac{1}{2}\left(\frac{\partial u_{\alpha}}{\partial x_{\beta}}+\frac{\partial u_{\beta}}{\partial x_{\alpha}}\right) \quad(\alpha, \beta=1,2,3)
$$

where $\alpha$ and $\beta$ represent the three possible directions of this motion.

Substituting Eqs. (47) and (48) into Eq. (50), the average strain tensor can be written in terms of the creation and annihilation operators as

$$
\varepsilon_{q}=i\left(\frac{\hbar \omega_{q}}{2 m v^{2}}\right)^{1 / 2}\left(b_{q}-b_{q}^{\dagger}\right)
$$

For optical phonons, the electron-phonon interaction is via the displacement along the normal mode coordinate, which is given in (48).

We can expand the electronic energy of the system with respect to the strain of each acoustic phonon mode and the normal mode coordinate of each optical phonon mode (the expansion series is truncated at the first order)

$$
E_{c}=E_{c, 0}+\sum_{q_{a}} a_{q, i-p} E_{c, 0} \varepsilon_{q}+\sum_{q_{o}} a_{q, i-p} E_{c, 0} Q_{q}
$$

where $E_{c, 0}$ is the static crystal field without any lattice displacement (before electronic transition) and $a_{q, i-p}$ is the ion-phonon coupling factor.

In the literature, $a_{q, i-p}$ is often determined by fitting to the experimental result, and so far no ab initio calculations have been made, to our knowledge. We plan to perform this ab initio calculation, in which we can determine the total energy before and after the transition $E_{c, 0}$ and $E_{c}$, as well as the lattice displacement vector $\boldsymbol{u}_{i}$. The strain $\varepsilon_{q}$ and the normal coordinate displacement $Q_{q}$ are then calculated using (50) and (48). These parameters can ultimately be substituted into (52) and the electron-phonon coupling factor $a_{q, i-p}$ is readily calculated for each phonon mode, including acoustic and optical modes. Our most recent results are reported in Ruan and Kaviany [133]. 


\section{Summary}

In summary, the ab initio calculations are powerful tools to investigate the energy carrier interactions at the atomic level. For a phonon-assisted absorption process described here, the ab initio calculations are capable of determining the lattice structure, electronic wave functions, and the total energy for the states before and after the electronic transition. Then, the electron-photon coupling is calculated using the wave functions, and the electron-phonon coupling by using the lattice displacement and energy modulation. These classes of couplings are ubiquitous in photon-electronphonon-coupled energy conversion processes, such as photovoltaic and lasers, and the ab initio approach described here can have broad impact on these systems.

\section{EXPLORING THE LIMITS OF THERMAL TRANSPORT IN NANOSTRUCTURED MATERIALS}

\section{Introduction}

Recent research on thermal transport in nanostructures has expanded the extremes of thermal transport in both the insulating and conducting limits. If we push the extremes far enough, the technological ramifications will be significant. Thermal management of integrated circuits is the biggest challenge in microelectronics today [134]. The most promising way to push magnetic data storage to $1 \mathrm{TBit} / \mathrm{in}^{2}$ is through thermally assisted magnetic recording in Co-based nanostructures [135]. Due to a phonon bottleneck, it is difficult to increase the frequency range of GaN microwave devices beyond $100 \mathrm{GHz}$ [136]. Ultralow thermal conductivity materials can be used for thermal barrier coatings in jet engines [137], whereas extremely high thermal conductivities can be used for heat sinking [138]. Also, if one can design materials with ultralow thermal conductivity and high electrical conductivity and thermopower, it may be possible to develop high-efficiency thermoelectric refrigerators and power generators [139]. This article reviews recent research that has broken the thermal transport limits by nanostructuring. It also proposes future research directions that could further push the extremes.

There are two types of heat carriers in solids: electrons and crystal vibrations (or phonons). This review focuses on the nonmetallic materials where phonons are the dominant carrier. A phonon is a quantum of crystal vibrational energy and is analogous to the photon [140]. Phonons have two fundamental lengths: its wavelength and mean free path. At room temperature, the dominant heat-carrying phonons typically have wavelengths of 1-3 nm and mean free paths of 10-100 nm. By using nanostructures comparable in size to these length scales, one can then manipulate thermal transport in solids [141, 142].

The schematic diagram in Figure 16 shows possible approaches to increase the range of thermal conductance and conductivity. The range of thermal conductivity at room temperature spans four orders of magnitude: from polymers at $\sim 0.1 \mathrm{~W} / \mathrm{m}-\mathrm{K}$ to diamond at $\sim 103 \mathrm{~W} / \mathrm{m}-\mathrm{K}$. Phonon transport in these materials is diffusive and the phonon scattering events are uncorrelated. In this article, we will focus on a broader range of phonon transport such as spectrally dependent scattering, phonon filtering, correlated scattering, wave guiding, and ballistic transport. In the case of phonon filtering and correlated scattering, it may be possible to prevent a spectral band(s) 


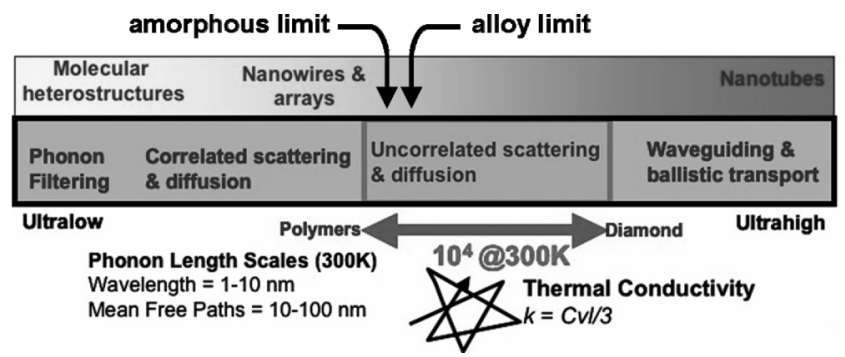

Figure 16. Schematic diagram illustrating various approaches to expand the limits of thermal transport.

from propagating, thereby reducing thermal conductivity. On the other hand, wave guiding and ballistic transport can increase the thermal conductance significantly.

\section{The Thermally Insulating Limit}

In this section, we review work that pushes the insulating limits in thermal transport. For example, we discuss how researchers beat the alloy and amorphous limits by nanostructuring. Recent reports on phonon filtering and correlated scattering are also reviewed.

The Alloy Limit The lowest thermal conductivity, $k$, in crystalline solids is generally that of an alloy and is often referred to as the alloy limit. Alloys have low thermal conductivity because the atomic substitutions heavily scatter phonons. This is known as alloy scattering. Thermal conductivities of alloys are typically $5<k<10$ $\mathrm{W} / \mathrm{m}-\mathrm{K}$ at room temperature. Historically, it has been difficult to beat the alloy limit without creating defects, dislocations, and voids. However, by using nanostructures such as superlattices [143, 144], nanowire [145], and nanoparticles [146, 147], researchers have been able to beat the alloy limit. In some cases [145, 147], these nanostructures provided a scattering mechanism other than alloy scattering such as spectrally dependent phonon scattering. In alloys, atomic substitutions scatter phonons due to differences in mass and/or bond stiffness. In the Rayleigh scattering regime, the scattering cross section varies as $\sigma \sim b^{6} / \lambda^{4}$ where $b$ is the size of the scattering particle and $\lambda$ is the phonon wavelength. Since atomic substitutions in alloys have $b \sim 1 \AA$, this relation suggests that alloys scatter short-wavelength phonons much more effectively than the mid- and long-wavelength phonons. Hence, these latter phonons dominate heat conduction in alloys. If one introduces another scattering mechanism that scatters mid- and/or long-wavelength phonons, the alloy limit can be beaten. Nanostructuring is one way of achieving this. Two examples are presented here-an alloy-based nanowire [145] and an alloy containing nanoparticles [147]. In nanowires, longwavelength phonons are scattered by the nanowire boundaries, i.e., boundary scattering [148], whereas in a bulk material containing nanoparticles, according to the acoustic Mie theory, mid- to long-wavelength phonons are scattered by nanoparticles [149-151] in the material.

Figure 17(a) shows thermal conductivities of 58- and 83-nm-diameter single crystalline $\mathrm{Si} / \mathrm{Si}_{x} \mathrm{Ge}_{1-x}$ superlattice nanowires and an $\mathrm{Si}_{0.9} \mathrm{Ge}_{0.1}$ alloy film for comparison [145]. The thermal conductivity of the nanowire is clearly below that of the alloy film. The thermal conductivity reduction is most pronounced in the low-temperature 

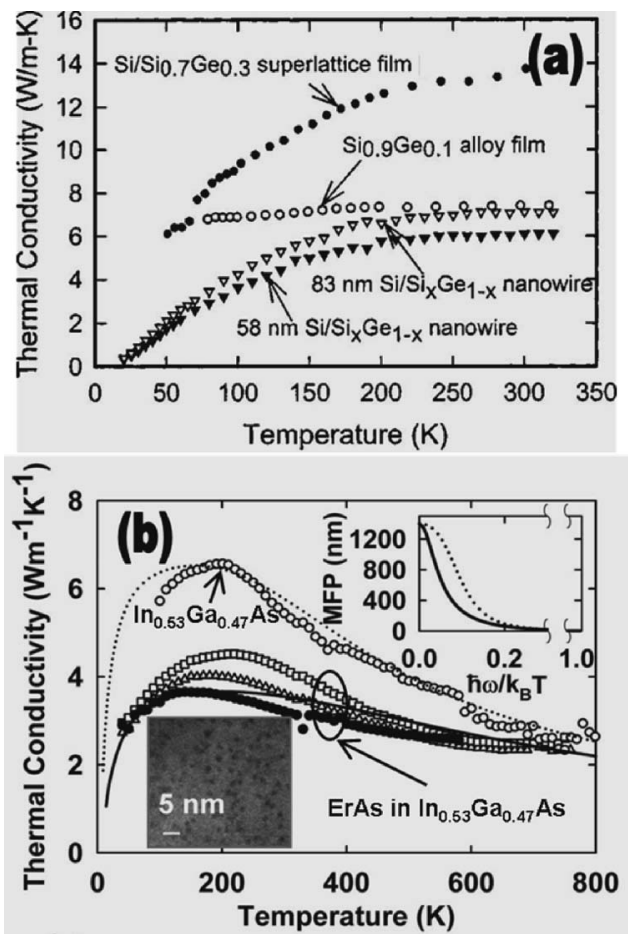

Figure 17. (a) Thermal conductivities of 58- and 83-nm-diameter single crystalline $\mathrm{Si} / \mathrm{Si}_{x} \mathrm{Ge}_{1-x}$ superlattice nanowires. The value of $x$ is $\sim 0.9-0.95$ and the superlattice period is $100-150 \mathrm{~nm}$. Thermal conductivities of a 30-nm period $2 \mathrm{D} \mathrm{Si} / \mathrm{Si}_{0.7} \mathrm{Ge}_{0.3}$ superlattice film and $\mathrm{Si}_{0.9} \mathrm{Ge}_{0.1}$ alloy film (3.5 $\mu \mathrm{m}$ thick) are also shown. (b) Thermal conductivity of randomly distributed ErAs in $\mathrm{In}_{0.53} \mathrm{Ga}_{0.47} \mathrm{As}$ (solid circles). Thermal conductivity of an $\operatorname{In}_{0.53} \mathrm{Ga}_{0.47}$ As alloy (open circles), $0.4 \mathrm{ML}$ with a 40 -nm period thickness $\mathrm{ErAs} / \mathrm{In}_{0.53} \mathrm{Ga}_{0.47} \mathrm{As}$ superlattice (open squares), and $0.1 \mathrm{ML}$ with a 10 -nm period thickness $\mathrm{ErAs} / \mathrm{In}_{0.53} \mathrm{Ga}_{0.47} \mathrm{As}$ superlattice (open triangles) are shown as references. Dotted and solid lines are based on theoretical analysis. The inset shows TEM pictures of randomly distributed ErAs in $\operatorname{In}_{0.53} \mathrm{Ga}_{0.47} \mathrm{As}$. Another inset shows the phonon mean free path (MFP) versus normalized frequency at $300 \mathrm{~K}$. Reprinted figure with permission from Kim et al. [147].

regime and negligible at room temperature. Recognizing that long-wavelength phonons dominate thermal transport only in the low-temperature regime, this thermal conductivity trend is a key signature of boundary scattering [140] by the $\mathrm{Si} / \mathrm{Si}_{x} \mathrm{Ge}_{1-x}$ superlattice nanowire.

Another example of beating the alloy limit by using spectrally dependent phonon scattering is shown in Figure 17(a). By introducing epitaxially embedded ErAs nanoparticles in a $\mathrm{In}_{0.53} \mathrm{Ga}_{0.47} \mathrm{As}$ alloy, the short-wavelength phonons are scattered by the $\mathrm{In}_{0.53} \mathrm{Ga}_{0.47} \mathrm{As}$ alloy and the mid- and long-wavelength phonons are scattered by the ErAs nanoparticles [147]. Figure 17(b) shows the thermal conductivity of $\mathrm{In}_{0.53} \mathrm{Ga}_{0.47} \mathrm{As}$ containing ErAs nanoparticles and an $\mathrm{In}_{0.53} \mathrm{Ga}_{0.47} \mathrm{As}$ alloy for comparison. Once again, the alloy limit is beaten by using nanostructures-ErAs nanoparticles. The inset compares the mean free path of ErAs nanoparticles in $\mathrm{In}_{0.53} \mathrm{Ga}_{0.47} \mathrm{As}$ with that of $\operatorname{In}_{0.53} \mathrm{Ga}_{0.47} \mathrm{As}$ versus phonon frequency at $300 \mathrm{~K}$. As shown in the inset, the mean free path of mid- to long-wavelength phonon has been 
suppressed, which indicates that ErAs nanoparticles preferentially scatter those phonon wavelengths.

The Amorphous Limit Amorphous solids do not exhibit long-range crystalline order [140]. This disorder destroys coherence of lattice vibrations and limits the mean free path to a few lattice spacings at most. This causes extremely low thermal conductivity and is often referred to as the amorphous limit. Thermal conductivities of amorphous oxides fall in the range $1.3<k<3.0 \mathrm{~W} / \mathrm{m}-\mathrm{K}$ at room temperatures [152].

Since the mean free path in an amorphous solid is already extremely small, beating this limit by further reducing the mean free path is not viable. Costescu et al. [152] beat the amorphous limit by using a material with a high interface density, the $\mathrm{W} / \mathrm{Al}_{2} \mathrm{O}_{3}$ nanolaminate. The $\mathrm{W} / \mathrm{Al}_{2} \mathrm{O}_{3}$ nanolaminate is a thin-film multilayer where each layer is only a few nanometers thick. In this material, they found that interfaces dominated the thermal conductance. The thermal conductivity of the $\mathrm{W} / \mathrm{Al}_{2} \mathrm{O}_{3}$ nanolaminate is shown in Figure 18. Data for an $\mathrm{Al}_{2} \mathrm{O}_{3}$ film, the calculated minimum conductivity, $k_{\text {min }}$, of $\mathrm{Al}_{2} \mathrm{O}_{3}$, and diffuse mismatch model (DMM) [153] analysis of the $\mathrm{W} / \mathrm{Al}_{2} \mathrm{O}_{3}$ nanolaminate are shown for comparison. As is clearly seen, the thermal conductivity of the $\mathrm{W} / \mathrm{Al}_{2} \mathrm{O}_{3}$ nanolaminate is lower than any other data.

Correlated Scattering Correlated scattering is defined as far-field interference of the scattered waves due to phase difference [150]. Scattering can be correlated in nanostructured materials. As Prasher suggested [150], when the distance between nanoparticles embedded in a material is comparable to the phonon wavelength, then scattering can be phase correlated. Therefore, one can manipulate the phonon mean free path by adjusting the inter-nanoparticle distance. Correlated scattering may also be important in nanowires. For example, the thermal conductivity of a thin 22-nm Si

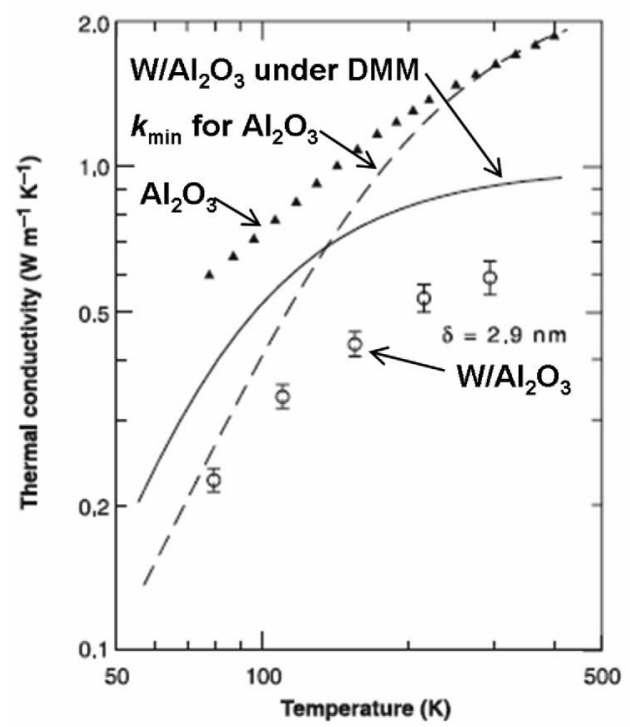

Figure 18. Temperature dependence of the thermal conductivity of the $\mathrm{W} / \mathrm{Al}_{2} \mathrm{O}_{3}$ nanolaminate with interface spacing, $\delta=2.9 \mathrm{~nm}$. Data for an $\mathrm{Al}_{2} \mathrm{O}_{3}$ film, the calculated minimum conductivity, $k_{\text {min }}$, of $\mathrm{Al}_{2} \mathrm{O}_{3}$, and diffuse mismatch model (DMM) [19] analysis of the $\mathrm{W} / \mathrm{Al}_{2} \mathrm{O}_{3}$ nanolaminate are shown for comparison. Reprinted figure with permission from Costescu et al. [152]. 
nanowire measured by Li et al. [148] had an unusual linear temperature dependence of thermal conductivity that cannot be explained by uncorrelated scattering [154] but could be explained by correlated scattering [155]. Murphy and Moore [155] numerically studied effects of coherence between multiple scattering events and found this linear temperature dependence in model systems.

Phonon Filtering Phonon filters prevent a spectral band(s) of phonons from propagating. The phonon filtering effect was first demonstrated by Narayanamurti et al. [156] in 1979. They showed selective transmission of high-frequency phonons at low temperature using $\mathrm{GaAs} / \mathrm{Al}_{0.5} \mathrm{Ga}_{0.5} \mathrm{As}$ superlattices. In the superlattice, phononic band gaps eliminate transmission of phonons that satisfy the Bragg condition. This approach provides a good way of preventing narrow bands of phonons from propagating (i.e., those that satisfy the Bragg condition). To improve insulation, phonon filters that prevent a wide band of phonons from propagating should be used. This can be done by using the spectral aspect of interface thermal transport. Generally speaking, only phonon frequencies that exist in both solids at an interface can transmit through that interface.

Interface thermal conductance is defined as $G=q / \Delta T$ where $q$ is the heat flux normal to the interface and $\Delta T$ is the temperature difference across the interface. The interface thermal conductance of metal-metal junctions [157] has a strong electronic contribution and is not discussed in this review. Prior to recent work [158, 159], measured vales of $G$ fell within a narrow range, approximately $30<G<175$ $\mathrm{MW} / \mathrm{m}^{2}-\mathrm{K}$ [160-163].

To create insulating interfaces, the dissimilarity in vibrational spectra of the interfacing materials should be maximized. This causes a lack of spectral coupling and minimizes the probability of phonon transmission across the interface. This effect is described by the diffuse mismatch model [153], which predicts the phonon transmission probability as a function of phonon frequency (see Figure 19). The dissimilarity in vibrational spectra is approximately proportional to the Debye temperature ratio of

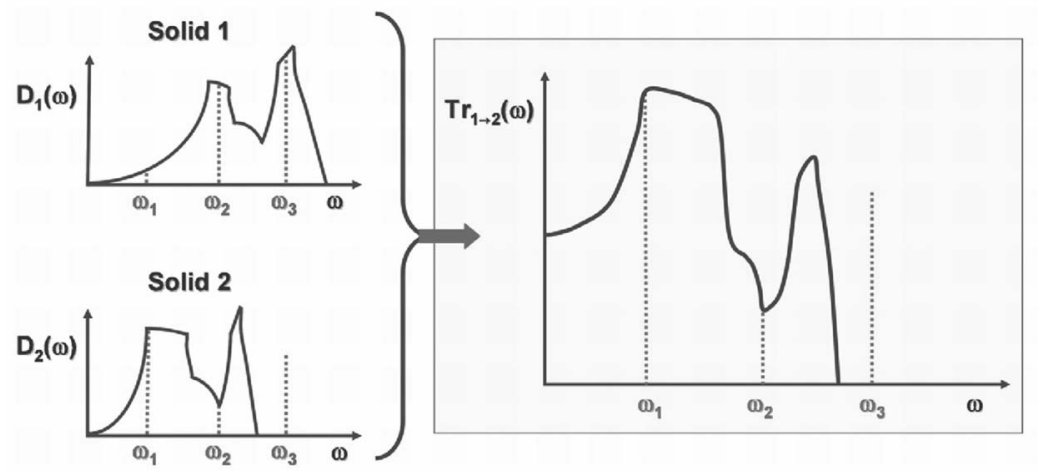

Figure 19. Illustration of the phonon transmission probability as predicted by the diffuse mismatch model [153]. Given two interfacing solids, 1 and 2 , with vibrational spectra, $D_{1}(\omega)$ and $D_{2}(\omega)$, the phonon transmission probability from 1 to $2, \operatorname{Tr}_{1 \rightarrow 2}(\omega)$ is dependent on the ratio of the spectra as shown. Representative locations of maxima and minima in the transmission probability are labeled on $\mathrm{D}_{1}(\omega)$, $\mathrm{D}_{2}(\omega)$, and $\operatorname{Tr}_{1 \rightarrow 2}(\omega)$. The transmission probability at $\omega_{3}$ is zero because solid 2 does not vibrate at that frequency. Since only the lower frequency phonons are transmitted, $\operatorname{Tr}_{1 \rightarrow 2}(\omega)$ is representative of a low-pass phonon filter. 


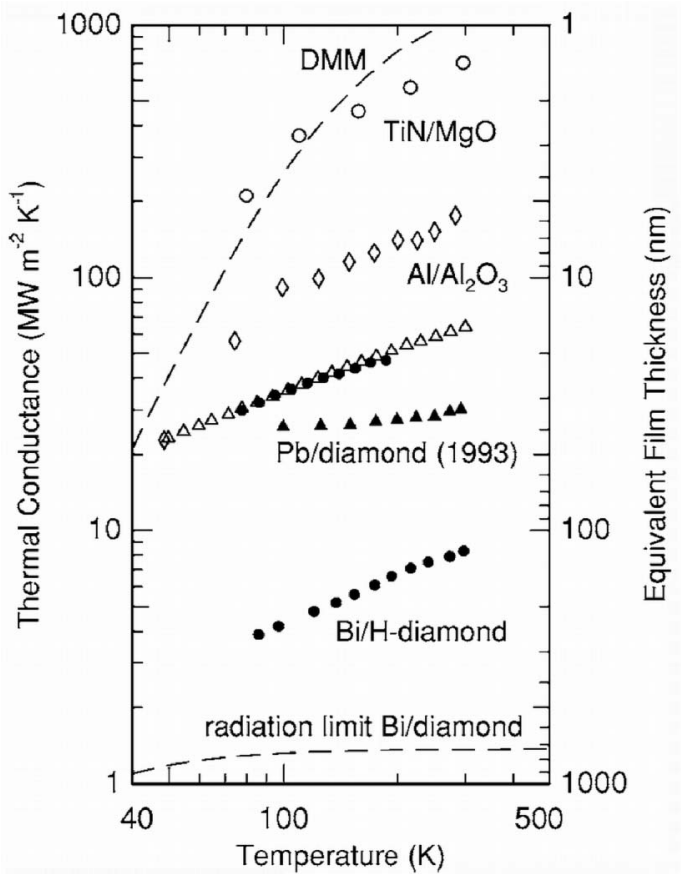

Figure 20. Compilation of the thermal conductance of solid-solid interfaces. The right-hand axis gives the equivalent thickness of a thin film with a thermal conductivity of $1 \mathrm{~W} / \mathrm{m}-\mathrm{K}$ that has the same thermal resistance as these single interfaces. Data are taken from Costescu et al. [158] (TiN/MgO, open circles); Stoner and Maris [160] ( $\mathrm{Al} / \mathrm{Al}_{2} \mathrm{O}_{3}$, open diamonds and $\mathrm{Pb} /$ diamond, filled triangles); Kim et al. [162] (GST/ZnS:SiO 2 , open triangles); Cahill et al. [163] (Al-oxide-Si structures, filled circles); Lyeo and Cahill [159] (Bi/hydrogen-terminated diamond, filled circles). The dashed line labeled "DMM" is a theoretical calculation of the TiN/MgO interface conductance using a modification of the diffuse-mismatch model; see Costescu et al. [158]. Reprinted figure with permission from Lyeo and Cahill [159].

the interfacing materials. Lyeo and Cahill [159] used materials with Debye temperature ratios of 5-20 to create interfaces with $8<G<30 \mathrm{MW} / \mathrm{m}^{2}-\mathrm{K}$. Figure 20 shows the current range of interface thermal conductance, which incorporates this new lower limit. The new upper limit on interface thermal conductance (discussed in Spectral Matching) is also shown.

This spectral aspect of interfacial thermal transport suggests that insulating interfaces can be made by phonon filtering. Phonon filters can insulate because they prevent propagation of a certain phonon band(s). For example, consider the insulating interface between $\mathrm{Pb}$ and diamond $[159,160]$. The dominant portions of the $\mathrm{Pb}$ [164] and diamond [165] vibrational spectra are in the $1-2 \mathrm{THz}$ and $50-80 \mathrm{THz}$ ranges, respectively. The interface is insulating because the diamond reflects the lowfrequency phonons of the $\mathrm{Pb}$ and the $\mathrm{Pb}$ reflects the high-frequency phonons of the diamond. Hence, $\mathrm{Pb}$ can be used as a low-pass filter and diamond can be used as a high-pass filter. It should be noted that in spectrally mismatched solids, the filtering of phonons is not perfect since anharmonic scattering enhances phonon transmission. This is demonstrated by the fact that the measured [159] Bi-diamond interface thermal conductance is greater than the radiation limit (see Figure). The radiation limit is the 


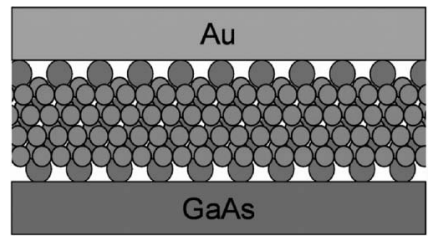

Figure 21. A molecular junction consisting of an alkanedithiol self-assembled monolayer sandwiched in between solid contacts of Au and GaAs. Sulfur, carbon, and hydrogen are represented by green, gray, and blue, respectively. The bonds along the chain are strong covalent bonds, whereas the lateral intermolecular bonds are weak van der Waals bonds. This molecular junction could exhibit band-pass phonon filtering and polarizing effects.

maximum possible interface conductance for a purely harmonic transport process involving one incoming and one outgoing phonon.

The existence of high- and low-pass phonon filters leads one to ask: Are other types of filters possible? A type of interface that may exhibit band-pass filtering is that of molecular junction. A molecular junction consists of a self-assembled monolayer sandwiched in between two solids (see Figure 21). Wang et al. [166] measured the thermal conductance of $\mathrm{Au} / \mathrm{GaAs}$ junctions with an interfacial alkanedithiol self-assembled monolayer (SAM). Even though the Debye temperatures ratio of $\mathrm{Au} / \mathrm{GaAs}$ is only $\sim 2$, the junction was insulating with $G \sim 25 \mathrm{MW} / \mathrm{m}^{2}-\mathrm{K}$. Considering that other junctions [167] with Debye temperature ratios of 2 have $\mathrm{G} \sim 100-200 \mathrm{MW} / \mathrm{m}^{2}-\mathrm{K}$, this molecular junction has a very low thermal conductance. This is likely caused by the SAM, which has a discrete vibrational spectra of narrow bands [168]. In comparison, $\mathrm{Au}$ [169] and GaAs [170] have a continuous broadband vibrational spectra. In this type of interface, only phonon bands resonant with the SAM's discrete vibrational spectra should transmit. Hence, the SAM acts as a band-pass filter.

In addition, molecular junctions such as SAMs should exhibit a phonon polarizing effect due to a contrast in bond strengths [166]. The lateral intermolecular bonds in a SAM are due to weak van der Waals interactions, whereas the Au and GaAs contain strong metallic and covalent bonds, respectively. In contrast, the bonds along the alkane backbone are covalent and similar in strength to the Au and GaAs bonds. Thus, the transverse vibrational frequencies of the SAM will be much lower than those of the Au and GaAs, whereas the longitudinal frequencies will be similar. This should cause preferential transmission of longitudinal vibrations and create a polarizing effect.

\section{The Thermally Conducting Limit}

In this section, we review work that pushes the conducting limits in thermal transport. For example, carbon nanotubes have already beaten the bulk limit of thermal conductivity. Experimental demonstrations of phonon wave guiding and ballistic phonon transport are also discussed.

Beyond Diamond-Carbon Nanotubes Prior to the emergence of carbon nanotubes (CNTs), diamond was regarded as the highest thermal conductor. Diamond's stiff $s p^{3}$ bonds result in a high phonon velocity and makes monocrystalline diamond one of the best thermal conductors [171]. The room-temperature thermal 
conductivity of naturally abundance diamond [172] is about $2300 \mathrm{~W} / \mathrm{m}-\mathrm{K}$, although that of isotopically pure diamond [173] is $3320 \mathrm{~W} / \mathrm{m}-\mathrm{K}$.

CNT possesses a unique combination of properties that make it an ideal thermal conductor. First, the carbon atoms in CNTs are bonded by $s p^{2}$ bonds, which are even strong than the $s p^{3}$ bonds in diamond [174]. Thus, phonon velocities are greater in CNTs than in diamond. Second, due to its seamless tubular structure, phonon boundary scattering is absent in CNTs. A remarkably high thermal conductance in CNTs has been calculated theoretically $[174,175]$ and demonstrated experimentally [176-181].

Before reviewing the thermal conductivity literature on individual CNTs, it should be mentioned that there is significant scatter in experimental data between different groups. This is especially true for multi-walled CNTs (MWCNTs), where the data can vary up to one order of magnitude. The fact that thermal conductivity can be affected by growth parameters [141] might be able to explain this. Figure 22 shows the thermal conductivities of an individual MWCNT [176] and individual single-walled [177] carbon nanotube (SWCNT). Pop et al. [181] also measured SWCNT thermal conductivity and found it to be $3500 \mathrm{~W} / \mathrm{m}-\mathrm{K}$ at room temperature, which is the highest room temperature value ever reported. The room temperature thermal conductivity of a MWCNT is 3128 $\mathrm{W} / \mathrm{m}-\mathrm{K}$ and is higher than that of naturally abundance diamond [172]. Although this is slightly less than isotopically pure diamond [173], that comparison may be irrelevant since the isotope content of the MWCNTs [176] was not measured.

Given the exceptionally large conductance of CNTs, many researches have tried to address whether thermal transport in CNTs is ballistic or diffusive. At present, the answer to this question is still unclear. The SWCNT measurement by Yu et al. [177] is very close to the calculated ballistic result for a 1-nm-diameter SWCNT and shows no characteristics of phonon-phonon Umklapp scattering from 110 to $300 \mathrm{~K}$. However, due to uncertainty in the SWCNT diameter, partially diffusive transport cannot be ruled out. In the case of MWCNTs, recent studies [182, 183] have observed ballistic

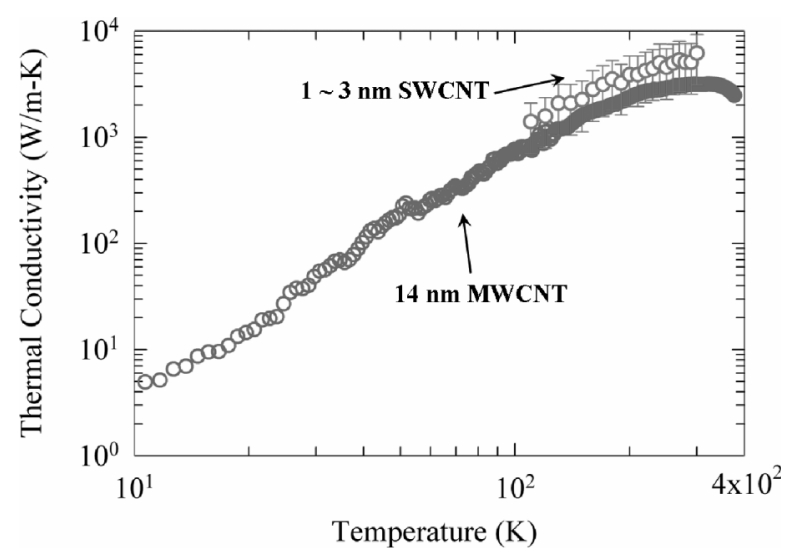

Figure 22. Thermal conductivities of an individual multi-walled carbon nanotube (MWCNT) and an individual single-walled carbon nanotube (SWCNT). Data for the MWCNT and SWCNT are from Kim et al. [176] and $\mathrm{Yu}$ et al. [177], respectively. An uncertainty in the SWCNT diameter created a large uncertainty in the SWCNT thermal conductivity. Reprinted data with permission from Kim et al. [176] and $\mathrm{Yu}$ et al. [177]. 
transport. However, data from Kim et al. [176] have an Umklapp scattering peak and is indicative of diffusive transport. Despite the ongoing debate on whether or not phonon transport is ballistic in CNTs, CNTs seem to be a potential candidate for phonon wave guiding.

The Quantum of Thermal Conductance Wave Guiding The quantum of thermal conductance, $g_{o}$, is the maximum possible value of thermal energy transported per phonon mode in a one-dimensional system [184]. The thermal conductance quantum is given by $\left(\pi^{2} k_{B}^{2} / 3 h\right) T$, where $k_{B}$ and $h$ are the Boltzmann and Planck constants, respectively. As shown in the expression, the quantum of thermal conductance is linearly dependent on temperature with slope $\pi^{2} k_{B}{ }^{2} / 3 h \approx 1 \mathrm{pW} / \mathrm{K}^{2}$.

The first measurement of the quantum of thermal conductance was reported by Schwab et al. [185] in 2000. The thermal conductance data is shown in Figure 23 and is normalized by $16 g_{0}$. Sixteen $g_{0}$ is physically significant because there are four phonon wave guides, each of which has only four occupied modes in the low-temperature limit. These four occupied modes correspond to one dilatational, one torsional, and two flexural degrees of freedom [185.]. Therefore, $16 g_{0}$ is the theoretical maximum value of thermal conductance in the low-temperature limit. As shown in the measured lowtemperature thermal conductance matches with the quantum of thermal conductance.

Spectral Matching In order to maximize interface conductance, materials with similar Debye temperatures and minimal intrinsic defects should be used. This was done by Costescu et al. [158], who measured the thermal conductance of epitaxial $\mathrm{TiN} / \mathrm{MgO}(001)$, TiN/MgO(111), and $\mathrm{TiN} / \mathrm{Al}_{2} \mathrm{O}_{3}(0001)$ junctions. Because these materials have similar Debye temperatures, the phonon filtering effect should be minimized. In addition, defects in the substrate and film were minimized by and careful fabrication procedures [158]. However, due to lattice mismatch and crystal orientations, the interfaces were expected to have varying amounts of misfit dislocations and stacking faults. For all three interfaces, the measured $G$ was approximately $700 \mathrm{MW} / \mathrm{m}^{2}-\mathrm{K}$. It is surprising that stacking faults and misfit dislocations did not have an effect on $G$. This may be an indication of other effects dominating $G$.

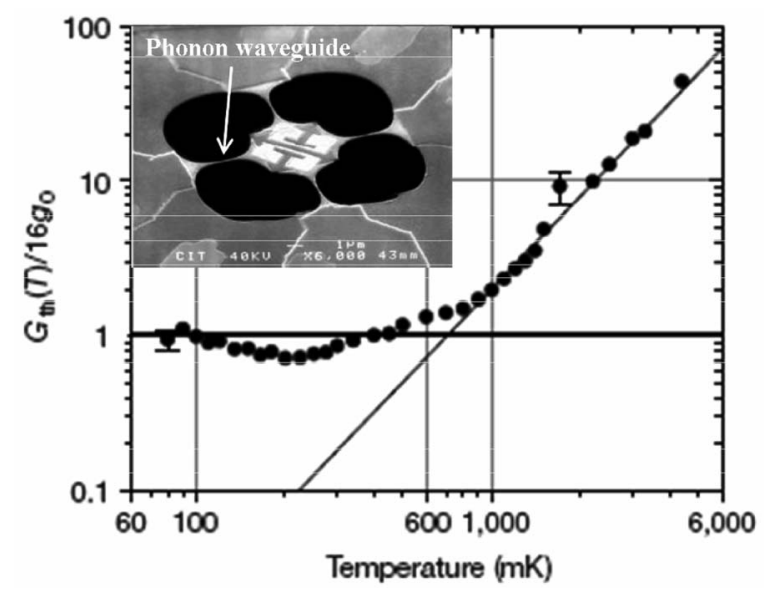

Figure 23. Measurement of the quantum of thermal conductance, $g_{0}$. The thermal conductance of the test structure is denoted by $G_{t h}$. Reprinted figure with permission from Schwab et al. [185]. 


\section{Summary}

These reviews show how limits in thermal transport can be broken by nanostructuring. In the thermally insulating limit, we discussed how spectrally dependent scattering has been used to beat the alloy limit. This was accomplished in both nanowires and an alloy with epitaxially embedded nanoparticles. By using a high interface density, the amorphous limit has also been beaten. Additionally, data on a thin Si nanowire thermal conductivity show linear temperature dependence, which may be indicative of correlated scattering.

Solid-solid junctions that exhibit high-pass and low-pass phonon filtering have been discussed. Furthermore, the low conductance of SAM interfaces may be indicative of band-pass phonon filtering. SAMs should also have a polarizing effect due to differences in inter- and intramolecular bond strengths.

In the thermally conducting limit, CNTs have been shown to beat the bulk limit of thermal conductivity. Despite the ongoing debate on whether or not phonon transport is ballistic in CNTs, CNTs seem to be a potential candidate for phonon wave guiding. Observation of the thermal conductance quantum opens up the possibility for extremely high thermal conductances. Lastly, spectral matching is suggested to enhance interface thermal conductance.

\section{REFERENCES}

1. W.G. Hoover, A.J.C Ladd, and V.N. Hoover, Historical Development and Recent Applications of Molecular Dynamics, ACS Advances in Chemistry Series, vol. 204, pp. 29-46, 1983.

2. W.G. Hoover, Molecular Dynamics, Springer Verlag, Berlin, 1986.

3. J.M. Haile, Molecular Dynamics Simulation, Elementary Methods, John Wiley \& Sons, New York, 1992.

4. D. Frenkel and B. Smit, Understanding Molecular Simulation, from Algorithms to Applications, 2nd ed., Academic Press, San Diego, 2002.

5. C.D. Holcomb, P. Clancy, and J.A. Zollweg, A Critical Study of the Simulation of the Liquid-Vapor Interface of a Lennard-Jones Fluid, Molecular Physics, vol. 78, pp. 437-459, 1993.

6. M. Chen, Z.-Y. Guo, and X.-G. Liang, Molecular Simulation of Some Thermophysical Properties and Phenomena, Microscale Thermophysical Engineering, vol. 5, pp. 1-16, 2001.

7. A.P. Wemhoff and V.P. Carey, Molecular Dynamics Exploration of Properties in the Liquid-Vapor Interfacial Region, Paper HT2003-47158, July 21-23, 2003; Las Vegas, Nev.

8. A.P. Wemhoff and V.P. Carey, Surface Tension Evaluation via Thermodynamic Analysis of Statistical Data from Molecular Dynamic Simulations, Paper HT-FED04-56690, July 14-16, 2004; Charlotte, N.C.

9. J. Alejandre, D.J. Tildesley, and G.A. Chapela, Molecular Dynamics Simulation of the Orthobaric Densities and Surface Tension of Water, The Journal of Chemical Physics, vol. 102, pp. 4574-4583, 1995.

10. L.X. Dang and T.-M. Chang, Molecular Dynamics Study of Water Clusters, Liquid, and Liquid-Vapor Interface of Water with Many-Body Potentials, The Journal of Chemical Physics, vol. 106, pp. 8149-8159, 1997.

11. M. Matusmoto, Y. Takaoka, and Y. Kataoka, Liquid-Vapor Interface of Water-Methanol Mixture. I. Computer Simulation, The Journal of Chemical Physics, vol. 98, pp. 1464-1472, 1993.

12. H. Daiguji, Molecular Dynamics Study of n-Alcohols Adsorbed on an Aqueous Electrolyte Solution, The Journal of Chemical Physics, vol. 115, pp. 1538-1549, 2001. 
13. J. Alejandre, D.J. Tildesley, and G.A. Chapela, Fluid Phase Equilibria Using Molecular Dynamics: The Surface Tension of Chlorine and Hexane, Molecular Physics, vol. 85, pp. 651-663, 1995.

14. A. Hariharan and J.G. Harris, J.G., Structure and Thermodynamics of the Liquid-Vapor Interface of Fluorocarbons and Semifluorinated Alkane Diblocks: A Molecular Dynamics Study, The Journal of Chemical Physics, vol. 101, pp. 4156-4165, 1994.

15. ASHRAE Fundamentals Handbook, American Society of Heating, Refrigeration and AirConditioning Engineers, Atlanta, GA, 2001.

16. K.S. Liu, Phase Separation of Lennard-Jones Systems: A Film in Equilibrium with Vapor, The Journal of Chemical Physics, vol. 60, pp. 4226-4230, 1974.

17. D.O. Dunikov, S.P. Malyshenko, and V.V. Zhakhovski, Corresponding States Law and Molecular Dynamic Simulations of the Lennard-Jones Fluid, The Journal of Chemical Physics, vol. 115, pp. 6623-6631, 2001.

18. A.P. Wemhoff and V.P. Carey, Molecular Dynamics Exploration of Thin Liquid Films on Solid Surfaces. 1. Monatomic Fluid Films, Microscale Thermophysical Engineering, vol. 9, pp. 331-349, 2005.

19. A.P. Wemhoff and V.P. Carey, Molecular Dynamics Exploration of Thin Liquid Films on Solid Surfaces. 2. Polyatomic Nonpolar Fluid and Water Films, Microscale Thermophysical Engineering, vol. 9, pp. 351-363, 2005.

20. A.P. Wemhoff and V.P. Carey, Disjoining Pressure Effects in Ultra-Thin Liquid Films in Micropassages - Comparison of Thermodynamic Theory with Predictions of Molecular Dynamics Simulations, IMECE2005-80234, November 5-11, 2005; Orlando, FL.

21. H. Goldstein, Classical Mechanics, Addison-Wesley, Reading, MA, 1950.

22. G.E.P. Box and M.E. Muller, A note on the Generation of Random Normal Deviates, Annual Mathematical Statistics, vol. 29, pp. 610-611, 1958.

23. G. Ciccotti and J.P. Ryckaert, Molecular Dynamics Simulation of Rigid Molecules, Computer Physics Reports, vol. 4, pp. 345-392, 1986.

24. I.C. Yeh and M.L. Berkowitz, Ewald Summation for Systems with Slab Geometry, The Journal of Chemical Physics, vol. 111, pp. 3155-3162, 1999.

25. J. Alejandre, D.J. Tildesley, and G.A. Chapela, Molecular Dynamics Simulation of the Orthobaric Densities and Surface Tension of Water, The Journal of Chemical Physics, vol. 102, pp. 4574-4583, 1995.

26. D.M. Heyes, Pressure Tensor of Partial-Charge and Point-Dipole Lattices with Bulk and Surface Geometries, Physical Review B, vol. 49, pp. 755-764, 1994.

27. R.M. Townsend, J. Gryko, and S.A. Rice, Structure of the Liquid-Vapor Interface of Water, The Journal of Chemical Physics, vol. 82, pp. 4391-4392, 1985.

28. J.-P. Hansen and I.R. McDonald, Theory of Simple Liquids, 2nd ed., Academic Press, London, 1986.

29. D. Frenkel and B. Smit, Understanding Molecular Simulation, from Algorithms to Applications, 2nd ed., Academic Press, San Diego, 2002.

30. Y. Chen, J.R. Lukes, D. Li, J. Yang, and Y. Wu, Thermal Expansion and Impurity Effects on Lattice Thermal Conductivity of Solid Argon, The Journal of Chemical Physics, vol. 120, pp. 3841-3846, 2004.

31. H.J.C. Berendsen, J.R. Grigera, and T.P. Straatsma, The Missing Term in Effective Pair Potentials, The Journal of Chemical Physics, vol. 91, pp. 6269-6271, 1987.

32. C. Andersen, Rattle: A "Velocity" Version of the Shake Algorithm for Molecular Dynamics Calculations, Journal of Computational Physics, vol. 52, pp. 24-34, 1983.

33. V.P. Carey and N.E. Hawks, Stochastic Modeling of Molecular Transport to an Evaporating Microdroplet in a Superheated Gas, Journal of Heat Transfer, vol. 117, pp. 432-439, 1995.

34. C.K. Oh, E.S. Oran, and R.S. Sinkovits, Computations of High-Speed, High Knudsen Number Microchannel Flows, Journal of Thermophysics and Heat Transfer, vol. 11, pp. 407-505, 1997. 
35. C. Mavriplis, J.C. Ahn, and R. Goulard, Heat Transfer and Flow Fields in Short Microchannels Using Direct Simulation Monte Carlo, Journal of Thermophysics and Heat Transfer, vol. 11, pp. 489-496, 1997.

36. R. Sayegh, M. Faghri, Y. Asako, and B. Sunden, Direct Simulation Monte Carlo of Gaseous Flow and Heat Transfer in a Microchannel, in M. Faghri and B. Sunden (eds.), Heat and Fluid Flow in Microscale and Nanoscale Structures, pp. 349-374, WIT Press, Southampton, UK, 2004.

37. P. Jiang and V.P. Carey, Parallelized DSMC Modeling of Transport Near Liquid-Vapor Interfaces in a Micro Bubble Heat Pipe, Paper IMECE2001/HTD-24194, November 11-16, 2001; ASME, New York, New York.

38. V.P. Carey, S.M. Oyumi, and S. Ahmed, Post Nucleation Growth of Water Microdroplets in Supersaturated Gas Mixtures: A Molecular Simulation Study, International Journal of Heat and Mass Transfer, vol. 40, pp. 2393-2406, 1997.

39. V.P. Carey, Surface Tension Effects on Post-Nucleation Growth of Water Microdroplets in Supersaturated Gas Mixtures, Journal of Heat Transfer, vol. 122, pp. 294-302, 2000.

40. V.P. Carey, DSMC Modeling of Interface Curvature Effects on Near-Interface Transport, Microscale Thermophysical Engineering, vol. 6, pp. 75-83, 2002.

41. G.A. Bird, Molecular Gas Dynamics and the Direct Simulation of Gas Flows, Oxford University Press, Oxford, UK, 1994.

42. R.C. Tolman, Effect of Droplet Size on Surface Tension, The Journal of Chemical Physics, vol. 17, pp. 333-343, 1949.

43. J.D. McDonald, A Computationally Efficient Particle Simulation Method Suited to Vector Computer Architectures, Report SUDAAR 589, December, 1989; Department of Aeronautics and Astronautics, Stanford University.

44. D. Baganoff and J.D. McDonald, A Collision-Selection Rule for a Particle Simulation Method Suited to Vector Computers, Physics of Fluids A, vol. 2, pp. 1248-1259, 1990.

45. H.R. Pruppacher and J.D. Klett, Microphysics of Clouds and Precipitation, D. Reidel Publishing Company, Dordrecht, Holland, 1980.

46. F. Peters and B. Paikert, Measurement and Interpretation of Growth and Evaporation of Monodispersed Droplets in a Shock Tube, International Journal of Heat and Mass Transfer, vol. 37, pp. 293-302, 1994.

47. V.P. Carey, Liquid-Vapor Phase Change Phenomena, Taylor \& Francis, New York, 1992.

48. F. Peters and K.A.J. Meyer, Measurement and Interpretation of Growth and Evaporation of Monodispersed Water Droplets Suspended in Pure Vapor, International Journal of Heat and Mass Transfer, vol. 38, pp. 3285-3293, 1995.

49. T. Ohara, Molecular Dynamics Simulation of Nanoscale Heat and Fluid Flow, in M. Faghri and B. Sunden (eds.), Heat and Fluid Flow in Microscale and Nanoscale Structures, pp. 349-374, WIT Press, Southampton, UK, 2004.

50. M. Planck, The Theory of Heat Radiation, Dover, New York, 1991.

51. G. Chen, Nanoscale Energy Transfer and Conversion, Oxford Press, Oxford, 2005.

52. M. Born and E. Wolf, Principles of Optics, Pergaman Press, Oxford, 1980.

53. R. Carminati and J.J. Greffet, Near Field Effects in Spatial Coherence of Thermal Sources, Physical Review Letters, vol. 82, pp. 1660-1663, 1999.

54. G. Chen and C.L. Tien, Partial Coherence Theory of Thin Film Radiative Properties, Journal of Heat Transfer, vol. 114, pp. 636-643, 1992.

55. L. Hu, A. Schmidt, A. Narayanaswamy, and G. Chen, Effects of Periodic Structures on the Coherence Properties of Blackbody Radiation, Journal of Heat Transfer, vol. 126, pp. 786-792, 2004.

56. P.W. Anderson, The Absence of Diffusion in Certain Random Lattices, Physical Review, vol. 109, pp. 1492-1505, 1958.

57. C.F. Bohren and D.R. Huffman, Absorption and Scattering of Light by Small Particles, Wiley, New York, 1983. 
58. A. Narayanaswamy, and G. Chen, Direct Computation of Thermal Emission from Nanostructures, Annual Review of Heat Transfer, vol. 14, pp. 169-196, 2005.

59. C. Luo, J.D. Joannopoulos, A. Narayanaswamy, and G. Chen, Thermal Radiation from Photonic Crystals: A Direct Calculation, Physical Review Letters, vol. 93, nos. 1-4, p. 213905, 2004.

60. E. Yablonovitch, Inihibited Spontaneous Emission in Solid-State Physics and Electronics, Physical Review Letters, vol. 58, pp. 2059-2062, 1986.

61. S.M. Rytov, Theory of Electric Fluctuations and Thermal Radiation, Paper \#AFCRC-TR59-162, 1959; Air Force Cambridge Research Center, Bedford, Mass.

62. D. Polder and M. Van Hove, Theory of Radiative Heat Transfer between Closely Spaced Bodies, Physical Review B, vol. 4, pp. 3303-3314, 1971.

63. A. Narayanaswamy and G. Chen, Thermal Radiation in 1D Photonic Crystals, Journal of Quantitative Spectroscopy and Radiative Transfer, vol. 93, pp. 175-183, 2005.

64. E.G. Cravolho, C.L. Tien, and R.P. Caren, Effect of Small Spacing on Radiation Transfer between Two Dielectrics, Journal of Heat Transfer, vol. 89, pp. 351-358, 1967.

65. H. Raether, Surface Plasmons on Smooth and Rough Surfaces and on Gratings, SpringerVerlag, Berlin, 1987.

66. A. Narayanaswamy and G. Chen, Surface Modes for Near-Field Thermophotovoltaics, Applied Physics Letters, vol. 82, pp. 3544-3546, 2003.

67. J.-J. Grefett, R. Carminati, K. Joulain, J.-P. Mulet, S. Malnguy, and Y. Chen, Coherent Emission of Light by Thermal Sources, Nature, vol. 416, pp. 61-64, 2002.

68. D.-Z.A. Chen, A. Narayanaswamy, and G. Chen, Surface Phonon-Polariton Mediated Thermal Conductivity Enhancement of Amorphous Thin Films, Physical Review B, vol. 72, 155435, 2005.

69. J.-P. Mulet, K. Joulain, R. Carminati, and J.-J. Greffet, Nanoscale Radiative Heat Transfer between a Small Particle and a Plane Surface, Applied Physics Letters, vol. 78, pp. 2931-2933, 2001.

70. J.B. Pendry, Negative Refraction Makes a Perfect Lens, Physical Review Letters, vol. 85, pp. 3966-3969, 2000.

71. D.R. Smith, J.B. Pendry, and M.C.K. Wiltshire, Metamaterials and Negative Refractive Index, Science, vol. 305, pp. 788-792, 2005.

72. B.J. Lee, C.J. Fu, and Z.M. Zhang, Coherent Thermal Emission from One-Dimensional Photonic Crystals, Applied Physics Letters, vol. 87, p. 071904-1/3. 2005.

73. M. Laroche, R. Carminati, and J.-J. Greffet, Coherent Thermal Antenna Using a Photonic Crystal Slab, Physical Review Letters, vol. 96, p. 123903/1-4, 2006.

74. L. Hu and G. Chen, unpublished.

75. R.G. Yang, A. Narayanaswamy, and G. Chen, Surface-Plasmon Coupled Nonequilibrium Thermoelectric Refrigerators and Power Generators, J. Computational and Theoretical Nanoscience, vol. 2, pp. 75-87, 2005.

76. D. Baüerle, Laser Processing and Chemistry, 3rd ed., Springer, Heidelberg, 2000.

77. E. Betzig, J.K. Trautman, T.D. Harris, J.S. Weiner, and R.L. Kostelak, Breaking the Diffraction Barrier: Optical Microscopy at Nanometric Scale, Science, vol. 251, pp. $1468-1470,1991$.

78. D.W. Pohl, W. Denk, and M. Lanz, Optical Stethoscopy: Image Recording with Resolution 入/20, Applied Physics Letters, vol. 44, pp. 651-653, 1984.

79. S. Wegscheider, A. Kirsch, J. Mlynek, and G. Krausch, Scanning Near-Field Optical Lithography, Thin Solid Films, vol. 264, pp. 264-267, 1995.

80. B. Dutoit, D. Zeisel, V. Deckert, and R. Zenobi, Laser-Induced Ablation through Nanometer-Sized Tip Apertures: Mechanistic Aspects, Journal of Physical Chemistry B, vol. 101, pp. 6955-6959, 1997.

81. K. Lieberman, Y. Shani, I. Melnik, S. Yoffe, and Y. Sharon, Near-field Optical Photomask Repair with a Femtosecond Laser, Journal of Microscopy, vol. 194, pp. 537-541, 1999. 
82. J. Jersch, F. Demming, and K. Dickmann, Nanostructuring with Laser Radiation in the Nearfield of a Tip from a Scanning Force Microscope, Applied Physics A, vol. 64, pp. 29-32, 1997.

83. S.M. Huang, M.H. Hong, Y.F. Lu, B.S. Lukyanchuk, W.D. Song, and T.C. Chong, Pulsed-Laser Assisted Nanopatterning of Metallic Layers Combined with Atomic Force Microscopy, Journal of Applied Physics, vol. 91, pp. 3268-3274, 2002.

84. J. Boneberg, H.J. Munzer, M. Tresp, M. Ochmann, and P. Leiderer, The Mechanism of Nanostructuring upon Nanosecond Laser Irradiation of a STM Tip, Applied Physics A, vol. 67, pp. 381-384, 1998.

85. A. Chimmalgi, T.-Y. Choi, C.P. Grigoropoulos, and K. Komvopoulos, Femtosecond Laser Apertureless Near-Field Nanomachining of Metals Assisted by Scanning Probe Microscopy, Applied Physics Letters, vol. 82, pp. 1146-1148, 2003.

86. A. Chimmalgi, C.P. Grigoropoulos, and K. Komvopoulos, Surface Nanostructuring by Nano-/Femtosecond Laser-Assisted Scanning Force Microscopy, Journal of Applied Physics, vol. 97, pp. 104319(1)-104319(12), 2005.

87. T.V. Pistor, Electromagnetic Simulation and Modeling with Applications in Lithography, Memorandum No. UCB/ERL M01/19, 2001; Berkeley, CA.

88. M. Ohtsu, Near-Field NanolAtom Optics and Technology, Springer-Verlag, Tokyo, 1998.

89. V.A. Ukraintsev and J.T. Yates, Nanosecond Laser Induced Single Atom Deposition with Nanometer Spatial Resolution Using a STM, Journal of Applied Physics, vol. 80, pp. 2561-2571, 1996.

90. Y. Kawata, C. Xu, and W. Denk, Feasibility of Molecular-Resolution Fluorescence NearField Microscopy Using Multi-Photon Absorption and Field Enhancement Near a Sharp Tip, Journal of Applied Physics, vol. 85, pp. 1294-1301, 1999.

91. R. Bachelot, F. H'Dhili, D. Barchiesi, G. Lerondel, R. Fikri, P. Royer, N. Landraud, J. Peretti, F. Chaput, G. Lampel, J. Boilot, and K. Lahlil, Apertureless Near-Field Optical Microscopy: A Study of the Local Tip Field Enhancement Using Photosensitive Azobenzene-Containing Films, Journal of Applied Physics, vol. 94, pp. 2060-2072, 2003.

92. J. Sanchez, L. Novotny, and X.S. Xie, Near-Field Fluorescence Microscopy Based on TwoPhoton Excitation with Metal Tips, Physical Review Letters, vol. 82, pp. 4014-4017, 1999.

93. T.W. Ebbesen, H.J. Lezec, H.F. Ghaemi, T. Thio, and P.A. Wolff, Extraordinary Optical Transmission through Sub-Wavelength Hole Arrays, Nature, vol. 391, pp. 667-669, 1998.

94. D.J. Hwang, A. Chimmalgi, and C.P. Grigoropoulos, Ablation of Thin Metal Films by Short-Pulsed Lasers Coupled through Near-Field Scanning Optical Microscopy Probes, Journal of Applied Physics, vol. 99, pp. 044905-044916, 2006.

95. C. Peng, L. Cheng, M. Mansuripur, Experimental and Theoretical Investigations of LaserInduced Crystallization and Amorphization in Phase-Change Optical Recording Media, Journal of Applied Physics, vol. 82, pp. 4183-4191, 1997.

96. A. Chimmalgi, D.J. Hwang, and C.P. Grigoropoulos, Nanoscale Rapid Melting and Crystallization of Semiconductor Thin Films, Nano Letters, vol. 5, pp. 1924-1930, 2005.

97. M. Lee, S. Moon, M. Hatano, K. Suzuki, and C.P. Grigoropoulos, Relationship between Fluence Gradient and Lateral Grain Growth in Spatially Controlled Excimer Laser Crystallization of Amorphous Silicon Films, Journal of Applied Physics, vol. 88, pp. 4994-4999, 2000.

98. P.A. Stolk, A. Polman, and W.C. Sinke, Experimental Test of Kinetic Theories for Heterogeneous Freezing in Silicon, Physical Review B, vol. 47, pp. 5-13, 1993.

99. M. Lee, S. Moon, and C.P. Grigoropoulos, In Situ Visualization of Interface Dynamics during the Double Laser Recrystallization of Amorphous Silicon Thin Films, Journal of Crystal Growth, vol. 226, pp. 8-10, 2001.

100. M.C. Wanke, O. Lehmann, K. Muller, W. Qingzhe, and M. Stuke, Laser Rapid Prototyping of Photonic Band-Gap Microstructures, Science, vol. 275, pp. 1284-1286, 1997.

101. D.J. Hwang, Pulsed Laser Processing of Electronic Materials in Micro/Nanoscale, Ph.D. Dissertation, University of California Berkeley, 2005. 
102. S. Chu, The Manipulation of Neutral Particles, Reviews of Modern Physics, vol. 70, pp. 685-706, 1998.

103. W. Phillips, Laser Cooling and Trapping of Neutral Atoms, Reviews of Modern Physics, vol. 70, pp. 721-741, 1998.

104. E. Cornell and C. Wieman, Nobel Lecture: Bose-Einstein Condensation in a Dilute Gas, the First 70 Years and Some Recent Experiments, Reviews of Modern Physics, vol. 74, pp. 875-893, 2002.

105. W. Ketterle, Nobel Lecture: When Atoms Behave as Waves: Bose-Einstein Condensation and the Atom Laser, Reviews of Modern Physics, vol. 74, pp. 1131-1151, 2002.

106. P. Pringsheim, Zwei Bemerkungen Uber den Unterschied von Lumineszenz und Temperaturstrahlung, Zeitschrift für Physik, vol. 57, pp. 739-746, 1929.

107. S. Vavilov, Some Remarks on the Stokes Law, Journal of Physics (Moscow), vol. 9, pp. 68-73, 1945.

108. S. Vavilov, Photoluminescence and Thermodynamics, Journal of Physics (Moscow), vol. 10, pp. 499-501, 1946.

109. L. Landau, On the Thermodynamics of Photoluminescence, Journal of Physics (Moscow), vol. 10, pp. 503-506, 1962.

110. C. Mungan, Radiation Thermodynamics with Applications to Lasing and Fluorescent Cooling, American Journal of Physics, vol. 73, pp. 1458-1460, 2005.

111. X.L. Ruan, S.C. Rand, and M. Kaviany, Entropy and Efficiency of Laser Cooling of Solids, Physical Review B, vol. 75, pp. 214304(1-9), 2007.

112. T. Kushida and J. Geusic, Optical Refrigeration in Nd-Doped Yttrium Aluminium Garnet, Physical Review Letters, vol. 21, pp. 1172-1175, 1968.

113. N. Djeu and W. Whitney, Laser Cooling by Spontaneous Anti-Stokes Scattering, Physical Review Letters, vol. 46, pp. 236-239, 1981.

114. R. Epstein, M. Buckwald, B. Edwards, T. Gosnell, and C. Mungan, Observation of LaserInduced Fluorescent Cooling of a Solid, Nature, vol. 377, pp. 500-503, 1995.

115. C. Mungan, M. Buchwald, B. Edwards, R. Epstein, and T. Gosnell, Internal Laser Cooling of $\mathrm{Yb}^{3+}$-Doped Glass Measured between 100 and $300 \mathrm{~K}$, Applied Physics Letters, vol. 71, pp. 1458-1460, 1997.

116. X. Luo, M. Eisaman, and T. Gosnell, Laser Cooling of a Solid by $21 \mathrm{~K}$ Starting from Room Temperature, Optics Letters, vol. 23, pp. 639-641, 1998.

117. B. Edwards, J. Anderson, R. Epstein, G. Mills, and A. Mord, Demonstration of a SolidState Optical Cooler: An Approach to Cryogenic Refrigeration, Journal of Applied Physics, vol. 86, pp. 6489-6493, 1999.

118. T. Gosnell, Laser Cooling of a Solid by $65 \mathrm{~K}$ Starting from Room Temperature, Optics Letters, vol. 24, pp. 1041-1043, 1999.

119. J. Fernandez, A. Mendioroz, A. Garcia, R. Balda, and J. Adam, Anti-Stokes LaserInduced Internal Cooling of $\mathrm{Yb}^{3+}$-Doped Glasses, Physical Review B, vol. 62, pp. 3213-3217, 2000.

120. R. Epstein, J. Brown, B. Edwards, and A. Gibbs, Measurements of Optical Refrigeration in Ytterbium Doped Crystals, Journal of Applied Physics, vol. 90, pp. 4815-4819, 2001.

121. A. Rayner, M. Friese, A. Truscott, N. Heckenberg, and H. Rubinsztein-Dunlop, Laser Cooling of a Solid from Ambient Temperature, Journal of Modern Optics, vol. 48, pp. 103-114, 2001.

122. C. Hoyt, M. Hasselbeck, M. Sheik-Bahae, R. Epstein, S. Greeneld, J. Thiede, J. Distel, and J. Valencia, Advances in Laser Cooling of Thulium-Doped Glass, Journal of the Optical Society of America B, vol. 20, pp. 1066-1074, 2003.

123. B. Heeg, M. Stone, A. Khizhnyak, G. Rumbles, G. Mills, and P. Debarber, Experimental Demonstration of Intracavity Solid-State Laser Cooling of $\mathrm{Yb}^{3+}$ : ZrF4-BaF2-LaF3AlF3-NaF Glass, Physical Review A, vol. 70, nos. 1-4, p. 021401, 2004.

124. J. Thiede, J. Distel, S. Greeneld, and R. Epstein, Cooling to $208 \mathrm{~K}$ by Optical Refrigeration, Applied Physics Letters, vol. 86, p. 154107, 2005. 
125. S. Bowman and C. Mungan, New Materials for Optical Cooling, Applied Physics B, vol. 71, pp. 807-811, 2000.

126. A. Mendioroz, J. Fernandez, M. Voda, M. Al-Saleh, R. Balda, and A. Garcia-Adeva, Anti-Stokes Laser Cooling in $\mathrm{Yb}^{3+}$-Doped $\mathrm{KPb}_{2} \mathrm{Cl}_{5}$ Crystal. Optics Letters, vol. 27, pp. 1525-1527, 2002.

127. M. Sheik-Bahae and R. Epstein, Can Laser Light Cool Semiconductors?, Physical Review Letters, vol. 368, p. 247403, 2004.

128. E. Merzbacher, Quantum Mechanics, 3rd ed., John Wiley, New York, 1998.

129. M. Hehlen, H. Gudel, Q. Shu, and S. Rand, Cooperative Optical Bistability in the Dimer System $\mathrm{Cs}_{3} \mathrm{Y}_{2} \mathrm{Br}_{9}: 10 \% \mathrm{Yb}^{3+}$, Journal of Chemical Physics, vol. 104, 1232-1244, 1996.

130. X. Ruan and M. Kaviany, Enhanced Laser Cooling of Rare-Earth-Ion-Doped Nanocrystalline Powders, Physical Review B, vol. 73, p. 155422(1-15), 2006.

131. B. Di Bartolo, Optical Interaction in Solids, John Wiley, New York, 1968.

132. R.C. Powell, Physics of Solid State Laser Materials, Springer, New York, 1998.

133. X.L. Ruan and M. Kaviany, Ab Initio Photon-Electron and Electron-Vibration Coupling Calculations in Laser Cooling of Ion-Doped Solids, Journal of Computational and Theoretical Nanoscience, in press, 2008.

134. R.C. Chu, R.E. Simons, M.J. Ellsworth, R.R. Schmidt, and V. Cozzolino, Review of Cooling Technologies for Computer Products, IEEE Transactions on Device Materials and Reliability, vol. 4, pp. 568-585, 2004.

135. T.W. McDaniel, Ultimate Limits to Thermally Assisted Magnetic Recording, Journal of Physics-Condensed Matter, vol. 17, pp. R315-R332, 2005.

136. S. Figge, T. Bottcher, D. Hommel, C. Zellweger, and M. Ilegems, Heat Generation and Dissipation in GaN-Based Light Emitting Devices, Physica Status Solidi A, vol. 200, pp. 83-86, 2003.

137. N.P. Padture, M. Gell, and E.H. Jordan, Materials Science-Thermal Barrier Coatings for Gas-Turbine Engine Applications, Science, vol. 296, pp. 280-284, 2002.

138. P.K. Schelling, L. Shi, and K.E. Goodson, Managing Heat for Electronics, Materials Today, vol. 8, pp. 30-35, 2005.

139. A. Majumdar, Thermoelectricity in Semiconductor Nanostructures, Science, vol. 303, pp. 777-778, 2004.

140. C.L. Tien, A. Majumdar, and F.M. Gerner, Microscale Energy Transport, Taylor \& Francis, Washington, DC, 1998.

141. D.G. Cahill, W.K. Ford, K.E. Goodson, G.D. Mahan, A. Majumdar, H.J. Maris, R. Merlin, and S.R. Phillpot, Nanoscale Thermal Transport, Journal of Applied Physics, vol. 93, pp. 793-818, 2003.

142. D.G. Cahill, K. Goodson, and A. Majumdar, Thermometry and Thermal Transport in Micro/Nanoscale Solid-State Devices and Structures, Journal of Heat TransferTransactions of the ASME, vol. 124, pp. 223-241, 2002.

143. W.S. Capinski, H.J. Maris, T. Ruf, M. Cardona, K. Ploog, and D.S. Katzer, Thermal Conductivity Measurements of GaAs/AlAs Superlattices Using a Picosecond Optical Pump-and-Probe Technique, Physical Review B, vol. 59, pp. 8105-8113, 1999.

144. R. Venkatasubramanian, Lattice Thermal Conductivity Reduction and Phonon Localizationlike Behavior in Superlattice Structures, Physical Review B, vol. 61, pp. 3091-3097, 2000.

145. D.Y. Li, Y. Wu, R. Fan, P.D. Yang, and A. Majumdar, Thermal Conductivity of Si/SiGe Superlattice Nanowires, Applied Physics Letters, vol. 83, pp. 3186-3188, 2003.

146. T.C. Harman, P.J. Taylor, M.P. Walsh, and B.E. LaForge, Quantum Dot Superlattice Thermoelectric Materials and Devices, Science, vol. 297, pp. 2229-2232, 2002.

147. W. Kim, J. Zide, A. Gossard, D. Klenov, S. Stemmer, A. Shakouri, and A. Majumdar, Thermal Conductivity Reduction and Thermoelectric Figure of Merit Increase by Embedding Nanoparticles in Crystalline Semiconductors, Physical Review Letters, vol. 96, p. 045901, 2006. 
148. D.Y. Li, Y.Y. Wu, P. Kim, L. Shi, P.D. Yang, and A. Majumdar, Thermal Conductivity of Individual Silicon Nanowires, Applied Physics Letters, vol. 83, pp. 2934-2936, 2003.

149. W. Kim and A. Majumdar, Phonon Scattering Cross Section of Polydispersed Spherical Nanoparticles, Journal of Applied Physics, vol. 99, p. 084306, 2006.

150. R. Prasher, Thermal Transport Due to Phonons in Random Nano-Particulate Media in the Multiple and Dependent (Correlated) Elastic Scattering Regime, Journal of Heat Transfer-Transactions of the ASME, vol. 128, pp. 627-637, 2006.

151. R.S. Prasher, Mie scattering Theory for Phonon Transport in Particulate Media, Journal of Heat Transfer-Transactions of the ASME, vol. 126, pp. 793-804, 2004.

152. R.M. Costescu, D.G. Cahill, F.H. Fabreguette, Z.A. Sechrist, and S.M. George, Ultra-Low Thermal Conductivity in W/Al $\mathrm{O}_{3}$ Nanolaminates, Science, vol. 303, pp. 989-990, 2004.

153. E.T. Swartz and R.O. Pohl, Thermal-Boundary Resistance, Reviews of Modern Physics, vol. 61, pp. 605-668, 1989.

154. N. Mingo, L. Yang, D. Li, and A. Majumdar, Predicting the Thermal Conductivity of Si and Ge Nanowires, Nano Letters, vol. 3, pp. 1713-1716, 2003.

155. P.G. Murphy and J.E. Moore, Phonon Localization by Coherent Multiple Scattering in Thermal Transport in Nanowires, unpublished.

156. V. Narayanamurti, H.L. Stormer, M.A. Chin, A.C. Gossard, and W. Wiegmann, Selective Transmission of High-Frequency Phonons by a Super-Lattice-Dielectric Phonon Filter, Physical Review Letters, vol. 43, pp. 2012-2016, 1979.

157. B.C. Gundrum, D.G. Cahill, and R.S. Averback, Thermal Conductance of Metal-Metal Interfaces, Physical Review B, vol. 72, p. 245426, 2005.

158. R.M. Costescu, M.A. Wall, and D.G. Cahill, Thermal Conductance of Epitaxial Interfaces, Physical Review B, vol. 67, p. 054302, 2003.

159. H.K. Lyeo and D.G. Cahill, Thermal Conductance of Interfaces between Highly Dissimilar Materials, Physical Review B, vol. 73, p. 144301, 2006.

160. R.J. Stoner and H.J. Maris, Kapitza Conductance and Heat-Flow between Solids at Temperatures from 50 to 300 K, Physical Review B, vol. 48, pp. 16373-16387, 1993.

161. A.N. Smith, J.L. Hostetler, and P.M. Norris, Thermal Boundary Resistance Measurements Using a Transient Thermoreflectance Technique, Microscale Thermophysical Engineering, vol. 4, pp. 51-60, 2000.

162. E.K. Kim, S.I. Kwun, S.M. Lee, H. Seo, and J.G. Yoon, Thermal Boundary Resistance at Ge2Sb2Te5/ZnS:SiO ${ }_{2}$ Interface, Applied Physics Letters, vol. 76, pp. 3864-3866, 2000.

163. D.G. Cahill, A. Bullen, and S.M. Lee, Interface Thermal Conductance and the Thermal Conductivity of Multilayer Thin Films, High Temperatures-High Pressures, vol. 32, pp. 135-142, 2000.

164. B.N. Brockhouse, K.R. Rao, A.D.B. Woods, G. Caglioti, and T. Arase, Crystal Dynamics of Lead .1. Dispersion Curves at 100 Degrees K, Physical Review, vol. 128, p. 1099, 1962.

165. J.L. Warren, J.L. Yarnell, G. Dolling, and R.A. Cowley, Lattice Dynamics of Diamond, Physical Review, vol. 158, p. 805, 1967.

166. R. Wang, R. Segalman, and A. Majumdar, Room Temperature Thermal Conductance of Alkanedithiol Self-Assembled Monolayers, Applied Physics Letters, vol. 89, p. 173113, 2006.

167. R.J. Stevens, A.N. Smith, and P.M. Norris, Measurement of Thermal Boundary Conductance of a Series of Metal-Dielectric Interfaces by the Transient Thermoreflectance Technique, Journal of Heat Transfer-Transactions of the ASME, vol. 127, pp. 315-322, 2005.

168. M.D. Porter, T.B. Bright, D.L. Allara, and C.E.D. Chidsey, Spontaneously Organized Molecular Assemblies .4. Structural Characterization of Normal-Alkyl Thiol Monolayers on Gold by Optical Ellipsometry, Infrared-Spectroscopy, and Electrochemistry, Journal of the American Chemical Society, vol. 109, pp. 3559-3568, 1987.

169. J.W. Lynn, H.G. Smith, and R.M. Nicklow, Lattice-Dynamics of Gold, Physical Review $B$, vol. 8, pp. 3493-3499, 1973. 
170. J.L.T. Waugh and G. Dolling, Crystal Dynamics of Gallium Arsenide, Physical Review, vol. 132, p. 2410, 1963.

171. L.H. Wei, P.K. Kuo, R.L. Thomas, T.R. Anthony, and W.F. Banholzer, ThermalConductivity of Isotopically Modified Single-Crystal Diamond, Physical Review Letters, vol. 70, pp. 3764-3767, 1993.

172. D.G. Onn, A. Witek, Y.Z. Qiu, T.R. Anthony, and W.F. Banholzer, Some Aspects of the Thermal-Conductivity of Isotopically Enriched Diamond Single-Crystals, Physical Review Letters, vol. 68, pp. 2806-2809, 1992.

173. T.R. Anthony, W.F. Banholzer, J.F. Fleischer, L.H. Wei, P.K. Kuo, R.L. Thomas, and R.W. Pryor, Thermal-Diffusivity of Isotopically Enriched C-12 Diamond, Physical Review B, vol. 42, pp. 1104-1111, 1990.

174. S. Berber, Y.K. Kwon, and D. Tomanek, Unusually High Thermal Conductivity of Carbon Nanotubes, Physical Review Letters, vol. 84, pp. 4613-4616, 2000.

175. N. Mingo and D.A. Broido, Carbon Nanotube Ballistic Thermal Conductance and Its Limits, Physical Review Letters, vol. 95, p. 096105, 2005.

176. P. Kim, L. Shi, A. Majumdar, and P.L. McEuen, Thermal Transport Measurements of Individual Multiwalled Nanotubes, Physical Review Letters, vol. 87, p. 215502, 2001.

177. C.H. Yu, L. Shi, Z. Yao, D.Y. Li, and A. Majumdar, Thermal Conductance and Thermopower of an Individual Single-Wall Carbon Nanotube, Nano Letters, vol. 5, pp. 1842-1846, 2005.

178. C.W. Chang, A.M. Fennimore, A. Afanasiev, D. Okawa, T. Ikuno, H. Garcia, D.Y. Li, A. Majumdar, and A. Zettl, Isotope Effect on the Thermal Conductivity of Boron Nitride Nanotubes, Physical Review Letters, vol. 97, p. 085901, 2006.

179. T.Y. Choi, D. Poulikakos, J. Tharian, and U. Sennhauser, Measurement of the Thermal Conductivity of Individual Carbon Nanotubes by the Four-Point Three-Omega Method, Nano Letters, vol. 6, pp. 1589-1593, 2006.

180. M. Fujii, X. Zhang, H.Q. Xie, H. Ago, K. Takahashi, T. Ikuta, H. Abe, and T. Shimizu, Measuring the Thermal Conductivity of a Single Carbon Nanotube, Physical Review Letters, vol. 95, p. 065502, 2005.

181. E. Pop, D. Mann, Q. Wang, K. Goodson, and H.J. Dai, Thermal Conductance of an Individual Single-Wall Carbon Nanotube above Room Temperature, Nano Letters, vol. 6 , pp. 96-100, 2006.

182. E. Brown, L. Hao, J.C. Gallop, and J.C. Macfarlane, Ballistic Thermal and Electrical Conductance Measurements on Individual Multiwall Carbon Nanotubes, Applied Physics Letters, vol. 87, p. 023107, 2005.

183. H.Y. Chiu, V.V. Deshpande, H.W.C. Postma, C.N. Lau, C. Miko, L. Forro, and M. Bockrath, Ballistic Phonon Thermal Transport in Multiwalled Carbon Nanotubes, Physical Review Letters, vol. 95, pp. 226101-4, 2005.

184. L.G.C. Rego and G. Kirczenow, Quantized Thermal Conductance of Dielectric Quantum Wires, Physical Review Letters, vol. 81, pp. 232-235, 1998.

185. K. Schwab, E.A. Henriksen, J.M. Worlock, and M.L. Roukes, Measurement of the Quantum of Thermal Conductance, Nature, vol. 404, pp. 974-977, 2000. 